\title{
British Thoracic Society guidelines for the management of non-tuberculous mycobacterial pulmonary disease (NTM-PD)
}

\author{
Charles S Haworth, ${ }^{1}$ John Banks, ${ }^{2}$ Toby Capstick, ${ }^{3}$ Andrew J Fisher, ${ }^{4}$ Thomas Gorsuch, $^{5}$ \\ Ian F Laurenson, ${ }^{6}$ Andrew Leitch, ${ }^{7}$ Michael R Loebinger, ${ }^{8}$ Heather J Milburn, ${ }^{9}$ \\ Mark Nightingale, ${ }^{10}$ Peter Ormerod, ${ }^{11}$ Delane Shingadia, ${ }^{12}$ David Smith, ${ }^{13}$ Nuala \\ Whitehead, ${ }^{14}$ Robert Wilson, ${ }^{8}$ R Andres Floto ${ }^{1,15}$
}

For numbered affiliations see end of article.

\section{Correspondence to} Dr Charles S Haworth Cambridge Centre for Lung Infection, Papworth Hospital, Cambridge CB23 3RE, UK; charles.haworth@nhs.net
CrossMark

To cite: Haworth CS, Banks J, Capstick T, et al. Thorax 2017;72:ii1-ii64.

\section{SUMMARY OF RECOMMENDATIONS AND GOOD PRACTICE POINTS}

\section{SECTION 4: WHAT IS THE EVIDENCE}

FOR TRANSMISSION OF NTM BETWEEN

INDIVIDUALS?

Recommendation

- Adequate infection control policies need to be implemented in both inpatient and outpatient settings to minimise risks of person-to-person transmission of Mycobacterium abscessus in individuals with cystic fibrosis (CF). (Grade B)

\section{SECTION 5: HOW SHOULD THE LUNG DISEASE} ATTRIBUTABLE TO NTM INFECTION BE DEFINED?

\section{Recommendation}

- In the absence of robust evidence to support an alternative definition and due to the clinical and research benefits of having a uniform definition, use of the American Thoracic Society/Infectious Diseases Society of America (ATS/IDSA) 2007 definition of non-tuberculous mycobacterial (NTM) pulmonary disease is recommended ${ }^{1}$ (see Box 1). (Grade D)

\section{Good practice point}

$\checkmark$ The management of coexisting lung conditions/ infections should be optimised before ascribing clinical decline to NTM-pulmonary disease.

\section{SECTION 6: WHAT SAMPLES SHOULD BE USED TO DETECT PULMONARY NON-TUBERCULOUS MYCOBACTERIAL INFECTION? Recommendations}

- Sputum, induced sputum, bronchial washings, bronchoalveolar lavage or transbronchial biopsy samples can be used to evaluate individuals suspected to have NTM-pulmonary disease. (Grade D)

- Whenever possible, less invasive sampling should be attempted first to minimise procedural risks. (Grade D)

- Respiratory samples should be processed within 24 hours of collection (or refrigerated at $4^{\circ} \mathrm{C}$ if delays are anticipated). (Grade D)
Oropharyngeal swab culture or serology testing should not be used to diagnose NTM-pulmonary infection. (Grade D)

\section{Good practice points}

$\checkmark$ Respiratory specimens should be collected with appropriate infection control precautions, including personal protective equipment, given the frequent differential diagnosis of $M$. tuberculosis infection. ${ }^{2}$

$\checkmark$ If sputum cultures are negative but clinical suspicion of NTM infection is high, consider performing CT-directed bronchial washings to obtain targeted samples.

$\checkmark \quad$ If individuals undergoing diagnostic evaluation for NTM infection are taking antibiotics that may impair NTM growth (such as aminoglycosides, macrolides, tetracyclines, co-trimoxazole, linezolid), consider discontinuing these antibiotics 2 weeks prior to collecting samples.

SECTION 7: WHAT MICROBIOLOGICAL TESTS SHOULD BE USED TO DETECT NTM IN RESPIRATORY SAMPLES?

Recommendations

- A validated rapid method should be used to detect NTM in respiratory samples. (Grade D)

- All respiratory samples should be stained using auramine-phenol after liquefaction and concentration and then examined by microscopy. (Grade B)

- Respiratory tract samples should be cultured (following decontamination) on solid and liquid media in a ISO15189-accredited clinical laboratory for 8 weeks extending to 12 if necessary. (Grade D)

- Routine use of non-culture-based detection methods is not recommended at the present time. (Grade D)

\section{Good practice point}

$\checkmark \quad$ If there is high clinical suspicion of NTM infection but negative sample cultures, consider 
discussing with a mycobacterial reference laboratory about (1) the possibility of culture on alternative media, at different temperatures, and/or for extended durations or (2) the utility of molecular detection methods.

\section{SECTION 8: WHAT MICROBIOLOGICAL TESTS SHOULD BE USED TO SPECIATE AND TYPE NTM FROM RESPIRATORY SAMPLES? \\ Recommendations}

- All NTM isolates from respiratory samples should be identified to at least species level using validated molecular or mass spectrometry techniques. (Grade B)

- Isolates of M. abscessus should be subspeciated using appropriate molecular techniques. (Grade $\mathrm{C}$ )

- If person-to-person transmission of M. abscessus is suspected, isolates should be typed, preferably using whole genome sequencing (Grade C).

\section{SECTION 9: DOES IN VITRO DRUG SUSCEPTIBILITY TESTING PREDICT RESPONSE TO ANTIBIOTIC TREATMENT IN PEOPLE WITH NTM-PULMONARY INFECTION? \\ Recommendations}

- Drug susceptibility testing and reporting should follow the Clinical Laboratory Standards Institute (CLSI) guidelines. (Grade D)

- For M. avium complex (MAC), clarithromycin and amikacin susceptibility testing should be performed on an isolate taken prior to initiation of treatment and on subsequent isolates if the patient fails to respond to treatment or recultures MAC after culture conversion. (Grade C)

- Macrolide-resistant MAC isolates should be tested against a wider panel of antibiotics to guide, but not dictate, treatment regimens. (Grade D)

- For M. kansasii, rifampicin susceptibility testing should be performed on an isolate prior to initiation of treatment and on subsequent isolates if the patient fails to respond to treatment or recultures M. kansasii after culture conversion. (Grade D)

- Rifampicin-resistant M. kansasii isolates should be tested against a wider panel of antibiotics to guide, but not dictate, treatment regimens. (Grade D)

- Susceptibility testing for M. abscessus should include at least clarithromycin, cefoxitin and amikacin (and preferably also tigecycline, imipenem, minocycline, doxycycline, moxifloxacin, linezolid, co-trimoxazole and clofazimine if a validated method is available) to guide, but not dictate, treatment regimens. (Grade D)

\section{Good practice points}

$\checkmark \quad$ Susceptibility testing should only be carried out on isolates where there is clinical suspicion of disease (to avoid unnecessary cost and conserve laboratory resources).

$\checkmark \quad$ Reporting of minimum inhibitory concentration (MIC) and critical concentration rather than susceptible or resistant may be more appropriate in the belief that a drug that has a very high MIC is unlikely to be active in vivo, whereas one just above a putative 'critical concentration' may have some activity, especially if combined with additive or synergistic agents.
SECTION 10: WHAT INVESTIGATIONS SHOULD BE PERFORMED IN PATIENTS SUSPECTED OF HAVING NTM-PULMONARY DISEASE?

Respiratory tract cultures

Recommendations

- A minimum of two sputum samples collected on separate days should be sent for mycobacterial culture when investigating an individual suspected of having NTM-pulmonary disease. (Grade D)

- Individuals suspected of having NTM-pulmonary disease whose sputum samples are consistently culture-negative for mycobacteria should have CT-directed bronchial washings sent for mycobacterial culture. (Grade D)

- Individuals suspected of having NTM-pulmonary disease who are unable to expectorate sputum should have CT-directed bronchial washings sent for mycobacterial culture. (Grade D)

- Transbronchial biopsies should not be performed routinely in individuals suspected of having NTM-pulmonary disease. (Grade D)

\section{Good practice points}

$\checkmark \quad$ Sputum induction resulting in a positive culture may avoid the need for CT-directed bronchial washings in individuals who are unable to spontaneously expectorate sputum.

$\checkmark$ Sputum induction should be considered in individuals suspected of having NTM-pulmonary disease who are unable to spontaneously expectorate sputum and in whom CT-directed bronchial washings are considered inappropriate.

\section{Radiology}

\section{Recommendations}

- A chest X-ray should be performed in individuals suspected of having NTM-pulmonary disease. (Grade D)

- A CT scan should be performed in individuals suspected of having NTM-pulmonary disease. (Grade D)

\section{Other investigations}

\section{Recommendations}

- There is insufficient evidence to recommend the routine use of serological testing for diagnosis and monitoring of individuals with NTM-pulmonary disease. (Grade D)

- Positron emission scanning, skin testing and interferon gamma release assays should not be used in the evaluation of individuals suspected of having NTM-pulmonary disease. (Grade D)

\section{SECTION 11: WHAT FACTORS INFLUENCE WHEN NTM TREATMENT SHOULD BE STARTED?}

\section{Recommendations}

- The decision to start treatment should be influenced by the severity of NTM-pulmonary disease, the risk of progressive NTM-pulmonary disease, the presence of comorbidity and the goals of treatment. (Grade D)

- Individuals may require a period of longitudinal assessment (symptoms, radiological change and mycobacterial culture results) to inform NTM treatment decisions. (Grade D)

\section{Good practice point}

$\checkmark$ The views of the affected individual should be sought on the potential risks and benefits of starting NTM treatment versus observation (ie, longitudinal assessment of symptoms, radiological change and mycobacterial culture results). 


\section{SECTION 12A: WHAT ANTIBIOTIC REGIMEN SHOULD BE USED TO TREAT MAC-PULMONARY DISEASE? Recommendations}

- Clarithromycin-sensitive MAC-pulmonary disease should be treated with rifampicin, ethambutol and clarithromycin or azithromycin using an intermittent (three times per week) or daily oral regimen. The choice of regimen should be based on the severity of disease (as defined in table 3) and treatment tolerance. (Grade D)

- An intermittent (three times per week) oral antibiotic regimen should not be used in individuals with severe MAC-pulmonary disease (as defined in table 3 ) or in individuals with a history of treatment failure. (Grade D)

- An injectable aminoglycoside (amikacin or streptomycin) should be considered in individuals with severe MAC-pulmonary disease (as defined in table 3). (Grade D)

- Clarithromycin-resistant MAC-pulmonary disease should be treated with rifampicin, ethambutol and isoniazid or a quinolone, and consider an injectable aminoglycoside (amikacin or streptomycin). (Grade D)

- Nebulised amikacin may be considered in place of an injectable aminoglycoside when intravenous/intramuscular administration is impractical, contraindicated or longer term treatment with an aminoglycoside is required for the treatment of MAC-pulmonary disease. (Grade D)

- Macrolide monotherapy or macrolide/quinolone dual therapy regimens should not be used for the treatment of MAC-pulmonary disease. (Grade D)

- Antibiotic treatment for MAC-pulmonary disease should continue for a minimum of 12 months after culture conversion. (Grade D)

\section{Good practice points}

$\checkmark \quad$ Individuals with clarithromycin-resistant MAC-pulmonary disease should be managed in collaboration with a physician experienced in managing NTM-pulmonary disease.

$\checkmark$ Individuals with a history of treatment intolerance or treatment failure should be managed in collaboration with a physician experienced in managing NTM-pulmonary disease.

\section{SECTION 12B: WHAT ANTIBIOTIC REGIMEN SHOULD BE USED TO TREAT M. KANSASII-PULMONARY DISEASE? \\ Recommendations}

- Rifampicin-sensitive M. kansasii-pulmonary disease should be treated with rifampicin, ethambutol and isoniazid or a macrolide (clarithromycin or azithromycin) using a daily oral regimen. (Grade D)

- Rifampicin-resistant M. kansasii-pulmonary disease should be treated with a three-drug regimen guided, but not dictated by, drug susceptibility test results using a daily oral regimen. (Grade D)

- Antibiotic treatment for M. kansasii-pulmonary disease should continue for a minimum of 12 months after culture conversion. (Grade D)

\section{Good practice points}

$\checkmark \quad$ Individuals with rifampicin-resistant M. kansasii-pulmonary disease should be managed in collaboration with a physician experienced in managing NTM-pulmonary disease.

$\checkmark \quad$ Individuals with a history of treatment intolerance or treatment failure should be managed in collaboration with a physician experienced in managing NTM-pulmonary disease.
SECTION 12C: WHAT ANTIBIOTIC REGIMEN SHOULD BE USED TO TREAT M. MALMOENSE-PULMONARY DISEASE? Recommendations

- M. malmoense-pulmonary disease should be treated with rifampicin, ethambutol and a macrolide (clarithromycin or azithromycin) using a daily oral regimen. (Grade D)

- An injectable aminoglycoside (amikacin or streptomycin) should be considered in individuals with severe $M$. malmoense-pulmonary disease (ie, acid-fast bacilli (AFB) smear-positive respiratory tract samples, radiological evidence of lung cavitation/severe infection or severe symptoms/signs of systemic illness). (Grade D)

- Nebulised amikacin may be considered in place of an injectable aminoglycoside when intravenous/intramuscular administration is impractical, contraindicated or longer term treatment with an aminoglycoside is required in the treatment of M. malmoense-pulmonary disease. (Grade D)

- Antibiotic treatment for M. malmoense-pulmonary disease should continue for a minimum of 12 months after culture conversion. (Grade D)

\section{Good practice points}

$\checkmark \quad$ Individuals with a history of treatment intolerance or treatment failure should be managed in collaboration with a physician experienced in managing NTM-pulmonary disease.

\section{SECTION 12D: WHAT ANTIBIOTIC REGIMEN SHOULD BE USED TO TREAT M. XENOPI PULMONARY DISEASE? \\ Recommendations}

- M. xenopi-pulmonary disease should be treated with a fourdrug regimen (where tolerated) comprising rifampicin, ethambutol and a macrolide (clarithromycin or azithromycin), with either a quinolone (ciprofloxacin or moxifloxacin) or isoniazid. (Grade D)

- An injectable aminoglycoside (amikacin or streptomycin) should be considered in individuals with severe M. xeno$p i$-pulmonary disease (ie, AFB smear positive respiratory tract samples, radiological evidence of lung cavitation/ severe infection or severe symptoms/signs of systemic illness). (Grade D)

- Nebulised amikacin may be considered in place of an injectable aminoglycoside when intravenous/intramuscular administration is impractical, contraindicated or longer term treatment with an aminoglycoside is required in the treatment of M. xenopi-pulmonary disease. (Grade D)

- Antibiotic treatment for M. xenopi-pulmonary disease should continue for a minimum of 12 months after culture conversion. (Grade D)

\section{Good practice point}

$\checkmark$ Individuals with a history of treatment intolerance or treatment failure should be managed in collaboration with a physician experienced in managing NTM-pulmonary disease.

\section{SECTION 12E: WHAT ANTIBIOTIC REGIMEN SHOULD BE USED TO TREAT $M$. ABSCESSUS-PULMONARY DISEASE? Recommendations}

- M. abscessus-pulmonary disease treatment should comprise an initial phase antibiotic regimen (including intravenous and oral antibiotics) followed by a continuation phase antibiotic regimen (including inhaled and/or oral antibiotics). (Grade D) 


\section{Initial phase}

- For individuals with M. abscessus isolates that are clarithromycin sensitive or demonstrate inducible macrolide resistance (see tables 7 and 8 ), the initial phase antibiotic regimen should include at least a 4-week course of intravenous amikacin, intravenous tigecycline, and (where tolerated) intravenous imipenem, and (where tolerated) oral clarithromycin or oral azithromycin. (Grade D)

- For individuals with M. abscessus complex isolates that demonstrate constitutive macrolide resistance (see tables 7 and 8 ), the initial phase antibiotic regimen should include a minimum 4-week course of intravenous amikacin, intravenous tigecycline and (where tolerated) intravenous imipenem. (Grade D)

- The duration of intravenous treatment should be influenced by the severity of infection, treatment response and tolerance of the regimen. (Grade D)

- To reduce the likelihood of treatment-related nausea and vomiting, antiemetic medication such as ondansetron (note potential for QT interval prolongation) and/oraprepitant should be prescribed to individuals receiving tigecycline and/ or imipenem. (Grade D)

- Nebulised amikacin may be considered in place of intravenous amikacin when intravenous administration is impractical, contraindicated or longer term treatment with an aminoglycoside is required in individuals with M. abscessus-pulmonary disease. (Grade D)

- In the context of amikacin-resistant M. abscessus (ie, MIC $>64 \mathrm{mg} / \mathrm{L}$ or the isolate is known to have a $16 \mathrm{~S}$ rRNA gene mutation conferring constitutive amikacin resistance), intravenous/nebulised amikacin should be substituted with an alternative intravenous/oral antibiotic. (Grade D)

\section{Continuation phase}

- For individuals with M. abscessus isolates that are clarithromycin-sensitive or demonstrate inducible macrolide resistance (see tables 7 and 8), the continuation phase antibiotic regimen should include nebulised amikacin and a macrolide (oral azithromycin or clarithromycin), in combination with one to three of the following oral antibiotics guided by drug susceptibility and patient tolerance: clofazimine, linezolid, minocycline or doxycycline, moxifloxacin or ciprofloxacin, and co-trimoxazole. (Grade D)

- For individuals with M. abscessus complex isolates that demonstrate constitutive macrolide resistance (see tables 7 and 8), the continuation phase antibiotic regimen should include nebulised amikacin in combination with two to four of the following oral antibiotics guided by drug susceptibility and patient tolerance: clofazimine, linezolid, minocycline or doxycycline, moxifloxacin or ciprofloxacin, and co-trimoxazole. (Grade D)

- In the context of amikacin-resistant M. abscessus (ie, MIC $>64 \mathrm{mg} / \mathrm{L}$ or the isolate is known to have a $16 \mathrm{~S}$ rRNA gene mutation conferring constitutive amikacin resistance), nebulised amikacin should be substituted with an alternative oral antibiotic. (Grade D)

- Antibiotic treatment for M. abscessus-pulmonary disease should continue for a minimum of 12 months after culture conversion. However, individuals who fail to culture-convert may benefit from a long-term suppressive antibiotic regimen. (Grade D)

\section{Good practice point}

$\checkmark \quad$ Individuals with M. abscessus-pulmonary disease should be managed in collaboration with a physician experienced in managing NTM-pulmonary disease.

\section{SECTION 13: IS THERE A ROLE FOR ADJUVANT THERAPIES IN THE MANAGEMENT OF NTM-PULMONARY DISEASE? Recommendations}

- Interferon gamma is not recommended as adjuvant therapy in individuals with NTM-pulmonary disease without a defined immunodeficiency affecting intrinsic interferon gamma signalling. (Grade D)

- M. vaccae is not recommended as adjuvant therapy in individuals with NTM-pulmonary disease. (Grade D)

\section{SECTION 14: WHAT INVESTIGATIONS SHOULD BE PERFORMED DURING TREATMENT OR FOLLOWING TREATMENT FOR NTM-PULMONARY DISEASE? Microbiological outcomes \\ Recommendations}

- Sputum samples should be sent for mycobacterial culture every 4-12 weeks during treatment and for 12 months after completing treatment to assess the microbiological response. (Grade D)

- If there is doubt about persisting NTM infection despite negative sputum cultures, a CT-directed bronchial wash should be performed to assess the microbiological response to treatment. (Grade D)

- In individuals who are unable to expectorate sputum, a CT scan followed by a CT-directed bronchial wash after 6 and 12 months of treatment can be used to assess the microbiological response to treatment. (Grade D)

\section{Good practice point}

$\checkmark \quad$ In individuals who are unable to spontaneously expectorate sputum and in whom CT-directed bronchial washings are not feasible, induced sputum samples should be sent for mycobacterial culture every 4-12 weeks during treatment and for 12 months after completing treatment to assess the microbiological response.

\section{Radiological outcomes}

\section{Recommendation}

- A CT scan should be performed shortly before starting NTM treatment and at the end of NTM treatment to document the radiological response to treatment. (Grade D)

\section{Good practice point}

$\checkmark$ During the course of treatment for NTM-pulmonary disease, more frequent radiological monitoring may be indicated in selected individuals.

\section{Clinical outcomes}

\section{Recommendation}

- A detailed assessment of pulmonary and systemic symptoms should be recorded at each clinical review. (Grade D)

\section{Good practice point}

$\checkmark$ A more detailed clinical assessment may include measurements of body weight, spirometry and systemic inflammatory markers (ESR and CRP). 


\section{Therapeutic drug monitoring}

Recommendations

- Therapeutic drug monitoring (other than for aminoglycosides) should not be performed routinely in individuals' prescribed antibiotic therapy for NTM-pulmonary disease. (Grade D)

- When aminoglycosides are administered, serum levels and the serum creatinine must be monitored and aminoglycoside dosing adjusted according to local policies. (Grade D)

\section{Good practice point}

$\checkmark \quad$ Therapeutic drug monitoring can be considered in individuals in whom gastrointestinal malabsorption, drug-drug interactions or suboptimal adherence may be adversely affecting treatment response.

\section{Monitoring for drug toxicity \\ Recommendations}

- When aminoglycosides are administered serum levels and the serum creatinine must be monitored and aminoglycoside dosing adjusted according to local policies. (Grade D)

- Audiometry should be considered before starting aminoglycosides and intermittently during treatment (frequency according to perceived risk and symptoms). Patients should be informed to stop aminoglycoside treatment immediately and to inform the prescriber if they develop tinnitus, vestibular disturbance or hearing loss. (Grade D)

- Assess visual acuity and colour vision before starting ethambutol and advise patients to stop treatment immediately and inform the prescriber if changes in visual acuity or colour vision occur. (Grade D)

- Serum ethambutol levels should be measured in patients with renal dysfunction. (Grade D)

\section{Good practice points}

$\checkmark \quad$ The frequency/type of toxicity monitoring required during NTM treatment is dependent on the drug regimen. Treatment-related adverse events and suggested toxicity monitoring protocols are outlined in the NTM antibiotic treatment monograph (section 18).

$\checkmark$ Audiometry should be considered before starting azithromycin or clarithromycin and intermittently during treatment (frequency according to perceived risk and symptoms) and advise individuals to stop treatment immediately and inform the prescriber if they develop tinnitus, vestibular disturbance or hearing loss.

$\checkmark$ Perform an ECG before, and 2 weeks after, starting drugs (such as azithromycin or clarithromycin) that are known to prolong the QT interval.

\section{SECTION 15: ARE THERE DIFFERENCES IN OUTCOME BETWEEN PATIENTS WITH NTM-PULMONARY DISEASE TREATED IN SPECIALIST VERSUS NON-SPECIALIST CARE SETTINGS?}

Recommendation

- Individuals with NTM-pulmonary disease should be managed in collaboration with a physician experienced in managing NTM-pulmonary disease. (Grade D)
SECTION 16: WHAT IS THE ROLE OF SURGERY IN THE TREATMENT OF NTM-PULMONARY

DISEASE?

\section{Recommendations}

- The role of lung resection surgery in the management of NTM-pulmonary disease should be considered at the time of diagnosis and revisited in individuals who develop refractory disease. (Grade D)

- Lung resection surgery for NTM-pulmonary disease may be indicated in individuals with localised areas of severe disease. (Grade D)

- Lung resection surgery for NTM-pulmonary disease should only be performed following expert multidisciplinary assessment in a centre experienced in managing individuals with NTM-pulmonary disease. (Grade D)

- Individuals with NTM-pulmonary disease should be established on antibiotic treatment prior to lung resection surgery and should continue treatment for 12 months after culture conversion. (Grade D).

- Following resection of a solitary NTM nodule in an individual with no other features of NTM-pulmonary disease, antibiotic treatment is not usually required. (Grade D)

\section{Good practice points}

$\checkmark$ Individuals with NTM-pulmonary disease in whom lung resection surgery is being considered should have a comprehensive assessment of cardiopulmonary status in line with current guidance for lung cancer resection.

$\checkmark \quad$ Nutritional status should be optimised prior to lung resection surgery.

\section{SECTION 17: DOES NTM INFECTION AFFECT AN INDIVIDUAL'S SUITABILITY FOR LUNG TRANSPLANTATION? \\ Recommendations}

- Individuals being considered for lung transplantation referral should be assessed for evidence of NTM-pulmonary disease. (Grade D)

- Isolation of NTM organisms including M. abscessus in potential lung transplant candidates should not preclude referral and assessment for lung transplantation. (Grade D)

- Potential lung transplant candidates with evidence of NTM-pulmonary disease should be treated whenever possible prior to listing to either eradicate the organism or lower bacterial load. (Grade D)

- Individuals with previous or current M. abscessus infection or disease who are listed for lung transplantation should be counselled about the high postoperative risk of developing invasive and disseminated NTM disease that causes significant morbidity and necessitates prolonged treatment with a multidrug antibiotic regimen. (Grade D)

\section{Good practice points}

$\checkmark$ Individuals with NTM-pulmonary disease should demonstrate an ability to tolerate optimal antibiotic therapy before listing for lung transplantation.

$\checkmark$ Progressive NTM-pulmonary disease despite optimal antibiotic therapy is likely to be a contraindication to listing for lung transplantation. 


\section{SECTION 1: INTRODUCTION Background}

Since the publication of the British Thoracic Society (BTS) Guideline on the 'Management of opportunistic mycobacterial infections' in 2000, our understanding of the epidemiology, microbiology and management of non-tuberculous mycobacterial-pulmonary disease (NTM-PD) has advanced. ${ }^{3}$ The incidence and prevalence of NTM-PD are increasing and are most likely explained by improved clinician awareness and enhanced detection methods, as well as a variety of changing environmental, mycobacterial and host factors. Technological advances in molecular microbiology have revolutionised our understanding of NTM taxonomy, and it is now appreciated that species and subspecies often differ in their pathogenicity and treatment response. While there remains a dearth of contemporary randomised controlled trial data to inform practice, the Guideline Development Group has sought to combine the best available evidence with clinical experience to create a pragmatic management guideline.

\section{Target audience for the guideline}

This guideline is aimed at healthcare practitioners who are involved in the care of individuals with NTM-PD, which will include hospital specialists in respiratory medicine, infectious diseases, paediatrics, microbiology immunology and radiology.

\section{Groups covered within the guideline}

a) individuals without pre-existing lung disease (de novo NTM infection)

b) individuals with COPD and other chronic inflammatory lung diseases

c) individuals with bronchiectasis

d) individuals with cystic fibrosis (CF)

e) individuals with a primary or secondary immunodeficiency

f) individuals being considered for and following lung transplantation.

\section{Groups not covered \\ - patients with extrapulmonary NTM disease \\ - neonates (birth to 28 days) and infants (1-12 months) \\ - patients with HIV infection.}

\section{Scope of the guideline}

1) epidemiology—incidence, prevalence and risk factors

2) microbiology-types of samples, detection and speciation

3) definition of NTM-PD and indications for treatment

4) antibiotic treatment regimens for specific NTM species

5) role of drug susceptibility testing (DST)

6) non-antibiotic treatment-interferon gamma, M. vaccae

7) investigation of individuals suspected of having NTM-PD and investigations to be undertaken during and after treatment

8) role of thoracic surgery

9) impact of NTM on lung transplant eligibility.

\section{SECTION 2: METHODOLOGY}

This guideline is based on the best available evidence. The methodology used to write the guideline adheres strictly to the criteria as set by the Appraisal of Guidelines for Research and Evaluation (AGREE) collaboration, which is available online www.agreetrust.org/resource-centre/agree-ii/. The BTS Standards of Care Committee (SOCC) guideline production manual is available at http://www.brit-thoracic.org.uk/ guidelines-and-quality-standards/.

\section{Clinical questions and literature search}

Clinical questions were structured in the PICO (Population, Intervention, Comparison, Outcome) format (online supplementary appendix 1) to define the scope of the guideline and inform the literature search.

Systematic electronic database searches were conducted in order to identify potentially relevant studies for inclusion in the guideline. For each topic area the following databases were searched: Ovid MEDLINE (including MEDLINE In Process), Ovid EMBASE and the Cochrane Library (including the Cochrane Database of Systematic Reviews, the Database of Abstracts of Reviews of Effects) from 1980.

The searches were first run in November 2012 and updated in June 2015 (see online supplementary appendix 1 for search strategy). Searches included a combination of indexed terms and free text terms and were limited to English-language publications only. The initial search identified 7186 potential abstracts and the second search 1687 abstracts.

\section{Appraisal of literature}

Appraisal was performed to be compliant with the AGREE collaboration. Two individuals (CSH, RAF) read the title and abstract of each article retrieved by the literature searches and decided whether the paper was definitely relevant, possibly relevant or not relevant to the project. Criteria formulated for categorising the abstracts into these three groups were the following:

- whether the study addressed the clinical question

- whether the appropriate study type was used to produce the best evidence to answer the clinical question

- review articles were excluded

- abstract was in English

- abstracts were not rejected on the basis of the journal of publication, country in which the research was performed or published, nor the date of publication.

The screened abstracts were allocated to the relevant section(s) of the guideline and two group members allocated to each guideline section. The full paper was obtained for all relevant or possibly relevant abstracts.

The first screening process identified 3443 of the initial 7186 reference abstracts to be definitely or possibly relevant to the guideline. Two guideline reviewers per section independently reviewed the abstracts to identify papers to be appraised for the guideline. The two reviewers for each section then independently appraised each paper assigned to them using the Scottish Intercollegiate Guidelines Network (SIGN) critical appraisal checklists. The reliability of the evidence in each individual study was graded using the SIGN critical appraisal checklists and is shown in the evidence tables $(++,+$ or -$)$. The body of evidence for each recommendation was summarised into evidence statements and graded using the SIGN grading system (see table 1).

Disagreements were resolved by discussion with the section partner. The second literature search in June 2015 yielded 1687 abstracts. Of these, 159 were identified as definitely or possibly relevant to the guideline. However, all of the pertinent ones from this search had been identified by the Guideline Development Group in the meantime and already incorporated.

\section{Considered judgement and grading of evidence}

The Guideline Development Group used the evidence tables to judge the body of evidence and grade recommendations for this guideline. Evidence tables (online supplementary appendix 2) are available online. Where evidence was lacking to answer 


\begin{tabular}{ll}
\hline Table 1 & Key to evidence statements \\
\hline Grade & Evidence \\
\hline $1++$ & $\begin{array}{l}\text { High-quality meta-analyses, systematic reviews of RCTs or RCTs with a } \\
\text { very low risk of bias }\end{array}$ \\
\hline $1+\quad \begin{array}{l}\text { Well-conducted meta-analyses, systematic reviews of RCTs or RCTs with a } \\
\text { low risk of bias }\end{array}$ \\
\begin{tabular}{ll} 
Meta-analyses, systematic reviews of RCTs or RCTs with a high risk of bias \\
$2++$ & $\begin{array}{l}\text { High-quality systematic reviews of case-control or cohort studies, or high- } \\
\text { quality case-control or cohort studies with a very low risk of confounding, } \\
\text { bias or chance, and a high probability that the relationship is causal }\end{array}$ \\
\hline $2+$ & $\begin{array}{l}\text { Well-conducted case-control or cohort studies with a low risk of } \\
\text { confounding, bias or chance, and a moderate probability that the } \\
\text { relationship is causal }\end{array}$ \\
\hline $2-$ & $\begin{array}{l}\text { Case-control or cohort studies with a high risk of confounding, bias or } \\
\text { chance, and a significant risk that the relationship is not causal }\end{array}$ \\
\hline 3 & $\begin{array}{l}\text { Non-analytic studies, for example, case reports, case series } \\
4\end{array}$ \\
\hline RCT, randomised opinion
\end{tabular} \\
\hline
\end{tabular}

the formulated clinical questions, expert opinions were obtained through consensus. The following were considered in grading of the recommendations:

- the available volume of the body of evidence

- how applicable the obtained evidence was in making recommendations for the defined target audience of this guideline

- whether the evidence was generalisable to the target population for the guideline

- whether there was a clear consistency in the evidence obtained to support recommendations

- what the implications of recommendations would be on clinical practice in terms of resources and skilled expertise

- cost-effectiveness was not reviewed in detail as in-depth economic analysis of recommendations falls beyond the scope of this guideline.

Recommendations were graded from $\mathrm{A}$ to $\mathrm{D}$ as indicated by the strength of the evidence as shown in table 2. In line with SIGN guidance, 'minus' evidence was considered in context but in the absence of other 'plus' supporting evidence, it was discussed among the GDG regarding that point and any

\begin{tabular}{ll}
\hline Table 2 & Grades of recommendations \\
\hline Grade & Type of evidence \\
\hline A & $\begin{array}{l}\text { At least one meta-analysis, systematic review or RCT rated as 1++ and } \\
\text { directly applicable to the target population or }\end{array}$ \\
& $\begin{array}{l}\text { A systematic review of RCTs or a body of evidence consisting principally } \\
\text { of studies rated as } 1+\text { directly applicable to the target population and } \\
\text { demonstrating overall consistency of results }\end{array}$ \\
\hline B & $\begin{array}{l}\text { A body of evidence including studies rated as } 2++ \text { directly applicable to } \\
\text { the target population and demonstrating overall consistency of results or }\end{array}$ \\
\hline C & $\begin{array}{l}\text { Extrapolated evidence from studies rated as 1++ or 1+ } \\
\text { target population and demonstrating overall consistency of results or }\end{array}$ \\
\hline D & $\begin{array}{l}\text { Extrapolated evidence from studies rated as } 2++ \\
\text { Evidence level } 3 \text { or } 4 \text { or }\end{array}$ \\
\hline$\sqrt{ }$ & $\begin{array}{l}\text { Extrapolated evidence from studies rated as } 2+ \\
\text { Important practical points for which there is no research evidence, nor is } \\
\text { there likely to be any research evidence. The guideline committee wishes } \\
\text { to emphasise these as good practice points. }\end{array}$ \\
\hline
\end{tabular}

recommendation hence made was Grade D. Important practical points lacking any research evidence nor likely to be research evidence in the future were highlighted as 'Good Practice Points' (GPP).

\section{Drafting the guideline}

The Guideline Development Group corresponded regularly by email, and meetings of the full group were held in May and July 2012, July and September 2013, January, April and November 2014, and June 2015, as well as a number of teleconferences. The Guideline Development Group was asked if they agreed or disagreed with the draft recommendations and GPP in an anonymous electronic survey administered by the BTS project coordinator in Spring 2016. The Guideline Development Group had agreed at the start of the guideline development process that $80 \%$ or more agreement would be the threshold for acceptance. Although not always unanimous, $80 \%$ or greater agreement was achieved for all recommendations and GPPs in the first round of voting.

The BTS SOCC reviewed the draft guideline in November 2016. The draft guideline was made available online in February 2017 for public consultation and circulated to all the relevant stakeholders. The BTS SOCC reviewed the revised draft guideline in June 2017 and final SOCC approval for publication was granted in July 2017.

This BTS Guideline will be reviewed within 5 years from publication date.

\section{Declarations of interest}

All members of the Guideline Group made declarations of interest in line with the BTS Policy and further details can be obtained on request from BTS.

\section{Guideline Development Group Members}

Dr Charles S Haworth, Co-Chair (Respiratory Medicine) (representing RCP London)

Professor R Andres Floto, Co-Chair (Respiratory Medicine)

Dr John Banks (Respiratory Medicine)

Dr Toby Capstick (Pharmacy)

Professor Andrew J Fisher (Respiratory Medicine)

Dr Thomas Gorsuch (Respiratory Medicine)

Dr Ian Laurenson (Scottish Mycobacteria Reference Laboratory)

Dr Andrew Leitch (Respiratory Medicine)

Dr Michael R Loebinger (Respiratory Medicine)

Professor Heather J Milburn (Respiratory Medicine)

Mr Mark Nightingale (Patient representative)

Professor L Peter Ormerod (Respiratory Medicine)

Dr Delane Shingadia (Paediatric Infectious Disease)

Dr David Smith (Respiratory Medicine)

Nuala Whitehead (Respiratory Nursing)

Professor Robert Wilson (Respiratory Medicine)

\section{Stakeholder organisations}

The following organisations were consulted during the public consultation period:

- Association of Respiratory Nurse Specialists

- Association of Chartered Physiotherapists in Respiratory Care

- British Infection Association

- British Lung Foundation

- British Paediatric Respiratory Society

- Royal College of Physicians of Edinburgh

- Royal College of Physicians, London. 


\section{SECTION 3: EPIDEMIOLOGY OF NTM-PULMONARY DISEASE Background}

The term non-tuberculous mycobacteria (NTM) refers to mycobacterial species other than the Mycobacterium tuberculosis complex (M. bovis, M. africanum, M. microti, M. canetti, $M$. caprae, $M$. pinnipedii, $M$ suricattae and $M$. mungi) and those organisms causing leprosy (M. leprae and M. lepromatosis). NTM are ubiquitous environmental organisms mostly found in soil and water and cause lung, sinus, lymph node, joint, CNS, and catheter-related and disseminated infections in susceptible individuals. $^{1}$

NTM can cause progressive inflammatory lung damage, a condition termed 'NTM pulmonary disease' (NTM-PD; described in section 5). However NTM can also transiently, intermittently or permanently reside within the lungs of individuals without causing NTM-PD, representing asymptomatic infection and creating considerable difficulties in deciding who and when to treat (see section 11).

NTM-PD can be broadly characterised as being either 'fibro-cavitary', (which is commonly mistaken for M. tuberculosis infection or malignancy, ${ }^{4}$ seen most often in current/ exsmokers, and often associated with smear-positive samples), or as 'nodular bronchiectatic' (most often seen in women with no previously identified lung disease ${ }^{5}$ where paucibacillary samples frequently cause diagnostic uncertainty ${ }^{4}$ ). National differences in reported gender ratios for NTM-PD are probably driven by differing proportions of these two types of lung disease. Thus, the USA, with more nodular-bronchiectatic than fibrocavitary disease, report more women than men with NTM-PD, ${ }^{6}$ while in Europe the situation is reversed. ${ }^{7}$

NTM are divided into slow-growing and rapid-growing species. The most common species causing lung infection are the slow-growing M. avium complex (MAC; consisting of M. avium, M. intracellulare and M. chimaera), M. kansasii, M. malmoense and M. xenopi, and the rapid-growing M. abscessus (consisting of M. a. abscessus, M. a. massiliense, M. a. bolletii subspecies), M. chelonae and M. fortuitum. ${ }^{17}$

These species vary in distribution between countries, ${ }^{7}$ within countries $^{8-22}$ and across different latitudes ${ }^{23}$ and water sources. ${ }^{24}$ Species also differ in their pathogenic potential. Thus, isolation of M. malmoense or M. abscessus is frequently indicative of NTM-driven inflammatory lung damage, whereas M. gordonae is rarely pathogenic and usually reflects sample contamination or transient colonisation. ${ }^{1}$

\section{Epidemiology}

Rates of NTM lung infection (which usually do not distinguish between individuals with NTM-positive cultures and those with defined NTM-PD) increase with age ${ }^{9}$ but vary considerably between countries. ${ }^{722} 25$ Most studies also report a rise in prevalence over the last four decades. ${ }^{61018-21} 2325-29$ Estimates from the USA suggest that the prevalence of NTM-positive cultures is currently between 1.4 and 6.6/100 000, ${ }^{6}$ while recent data from the UK suggest that the incidence of pulmonary NTM-positive cultures increased from $4.0 / 100000$ in 2007 to $6.1 / 100000$ in 2012. ${ }^{30}$

Although improved detection methods and enhanced physician awareness could explain some of the observed increased in NTM-positive cultures, a number of strands of evidence suggests a real underlying increase: a report from Canada demonstrated a 5-year increase in prevalence of NTM-pulmonary disease of 29.3 cases/100 000 in 1998-2002 to 41.3/100 000 in 2006$2010(\mathrm{p}<0.0001)$, and found that improved laboratory methods did not explain this ${ }^{29}$; a number of CF studies (eg, ref ${ }^{31}$ ) show year-on-year increases in NTM-positive cultures with no change in surveillance intensity or culture methodology; an increase over time in rates of skin test reactivity to NTM antigens in US population-based studies, ${ }^{32}$ most likely indicating increasing exposure to NTM (see below).

Possible causes driving a real increase in NTM infection rates include declining rates of $M$. tuberculosis infection potentially reducing population immunity to mycobacteria ${ }^{33}$; increases in environmental exposure to NTM through more permissive temperature settings of home water heaters ${ }^{34}$ and more contact with shower aerosols ${ }^{35-37}$; increased long-term antibiotic usage in inflammatory lung diseases, potentially creating a more favourable lung niche for $\mathrm{NTM}^{38}$; greater use of medications that might impair host immunity to NTM (see below) ${ }^{31}$; and the potential impact of person-to-person transmission of certain NTM species in some patient cohorts (eg, in CF). ${ }^{39-41}$

\section{Risk factors for NTM-pulmonary disease}

Most defined risk factors for NTM-PD can be grouped into those that influence either NTM exposure or host susceptibility and are summarised below.

\section{Exposure}

Studies have suggested that, for any given level of host susceptibility, there might be a threshold exposure level for NTM associated with the development of disease. Thus, some, but not all, studies show associations with activities potentially increasing exposure to NTM in water (swimming), soils (gardening) and aerosols (showering, hot tubs). ${ }^{35}$ In a study from Queensland, researchers were able to match NTMs isolated from soil and water in the home to clinical isolates from infected patients, ${ }^{37}$ underpinning the likely importance of the local environment.

The wide variation in NTM rates across different US states ${ }^{9}{ }^{10}$ correlated with different levels of evapotranspiration (the process of converting surface water to atmospheric water vapour) and the area covered by surface water, ${ }^{9} 10$ while other studies have shown geographical associations of NTM infection with lower lying areas, greater levels of precipitation, the source of potable water, and greater mean, maximum and minimum temperatures. ${ }^{19} 42-44$

\section{Host susceptibility}

\section{Pre-existing lung disease}

NTM infection is more common in individuals with chronic lung diseases such as asthma ${ }^{45}$ COPD, ${ }^{28} 4647$ alpha-1 antitrypsin deficiency, ${ }^{48}$ pneumoconiosis, ${ }^{49}{ }^{50} \mathrm{CF}^{51}{ }^{52}$ non-CF bronchiectasis, ${ }^{53-55}$ primary ciliary dyskinesia ${ }^{56}$ and allergic bronchopulmonary aspergillosis, ${ }^{57} 58$ which may predispose to NTM infection by causing chronic epithelial cell inflammation and impaired mucociliary clearance. A recent study has suggested that NTM-infected individuals with no obvious pre-existing lung condition may have quantitatively impaired ciliary function. ${ }^{59}$ NTM infection is also more common in individuals heterozygous for CFTR mutations, ${ }^{6061}$ suggesting that subtle changes in control of airway surface liquid may also be critical in determining host susceptibility.

\section{Lady Windermere syndrome}

Many studies, predominantly US-based, have described a series of characteristic features of patients with nodular-bronchiectatic 
NTM-PD who were more likely to be white, postmenopausal, thin, tall women with pectus excavatum and mitral valve prolapse who develop predominantly middle lobe and lingula bronchiectasis; a cluster of features described as 'Lady Windermere Syndrome', ${ }^{52-67}$ after the eponymous heroine of the Oscar Wilde play. These individuals do not have pre-existing lung disease and are usually non-smokers. Recently, whole exome sequencing has begun to define genetic predispositions for this type of presentation, ${ }^{67}$ highlighting genes involved in immune regulation, cilial function and connective tissue.

\section{Other comorbidities}

A number of other comorbidities have been suggested to potentially contribute to NTM acquisition, including the presence of gastro-oesophageal reflux disease, ${ }^{68} 69$ rheumatoid arthritis, ${ }^{70-72}$ low vitamin $\mathrm{D},{ }^{73}$ low body mass index (BMI) and malnutrition. $^{12646675-78}$

\section{Immunodeficiency}

A number of immunodeficiencies have been associated with NTM infection, ${ }^{79} 80$ including inherited disorders of IFN $\gamma$-IL12 pathway (eg, IFN $\gamma \mathrm{R} 1$ mutations), ${ }^{81}$ other cytokine signalling (eg, STAT mutations), ${ }^{82}$ and macrophage and dendritic cell function (eg, GATA2 ${ }^{83}$ NRAMP1 ${ }^{84}$ ), as well as acquired immunodeficiencies including HIV-AIDS ${ }^{85}$ and functional anti-interferon gamma antibodies. ${ }^{86}$ Although frequently presenting with disseminated NTM infection, immunodeficiencies should also be considered in individuals with recurrent, persistent or severe pulmonary infection, particularly with NTM species that are normally non-pathogenic, as well as in those presenting with NTM disease in the absence of structural lung disease, or in those presenting at a young age. In these cases referral to a clinical immunologist is recommended.

\section{Medications}

Immunosuppression

A number of different immunosuppressive drugs have been associated with acquisition of NTM infection: oral as well as inhaled corticosteroids, ${ }^{45} 4987$ anti-tumour necrosis factor (TNF)- $\alpha$ therapy $^{88}$ (consistent with its known effects on host immunity to mycobacteria), combination immunosuppression used in solid organ transplantation ${ }^{85}$ and immunosuppression associated with cancer chemotherapy. ${ }^{85}$

\section{Azithromycin}

In a single-centre study of adults with CF, Renna et al reported increases in annual rates of NTM infection associated with chronic azithromycin use, suggesting through in vitro and mouse infection studies that azithromycin, at therapeutic doses, could block autophagic killing of NTM within macrophages. ${ }^{31}$ While supported by epidemiological findings from a previous casecontrol in Israel $^{43}$ and a recent European CF Society Patient Registry study, ${ }^{89}$ other large retrospective studies have shown no such association, potentially highlighting the complex relationship between the antibiotic and immunomodulatory properties of azithromycin during NTM infection..$^{90-95}$

\section{Inhaled antibiotics}

Several studies of individuals with CF have shown an association of inhaled antibiotic use with NTM infection, ${ }^{38} 89$ suggesting that other bacterial species may, in the absence of inhaled antibiotics, outcompete NTM for suitable lung niches.
Proton pump inhibitors

The impact of proton pump inhibitors (PPI) is unclear. PPI use has been reported to be associated with the development of MAC-pulmonary disease ${ }^{68}$ and may promote gastrointestinal survival of NTM and subsequent lung infection through gastric aspiration.

\section{SECTION 4: WHAT IS THE EVIDENCE FOR TRANSMISSION OF NTM BETWEEN INDIVIDUALS? \\ Evidence summary}

Person-to-person transmission of NTM has traditionally been considered unlikely. During the HIV epidemic, typing of MAC isolates indicated that individuals acquired their infection from environmental sources rather than from each other. Similarly, extensive work on M. avium infection indicates environmental point sources rather than animal to human transmission as the likely mechanism of acquisition. ${ }^{96-98}$ Within people with CF, two separate studies (based on data from 1990s and 2000s), showed that patients, even siblings living in the same household for more than 10 years, had unique strains of NTM, ${ }^{99} 100$ suggesting a lack of person-person transmission.

However, a case report from the University of Washington, Seattle, USA, described a possible outbreak of M. a. massiliense in five individuals with $\mathrm{CF}^{101}$ with potential transmission occurring during synchronous clinic visits. More recently, whole genome sequencing, detailed epidemiology and antimicrobial susceptibility testing performed on 168 consecutive isolates of M. abscessus from 31 patients attending an adult CF centre in the UK revealed frequent, probably indirect, transmission of $M$. a. massiliense between individuals with CF (and, in one case, an individual with non-CF bronchiectasis) despite conventional cross-infection prevention measures. ${ }^{40}$ Whole genome sequencing of the Seattle centre outbreak confirmed the presence of near-identical isolates shared between patients. ${ }^{102}$ A larger, multinational whole genome sequence analysis of over 1000 clinical isolates of M. abscessus from individuals with $\mathrm{CF}^{41}$ indicates widespread patient-to-patient transmission of M. abscessus with evidence of transcontinental spread of dominant circulating clones, which may be associated with worse clinical outcome and are more virulent when tested experimentally. Although a small study from the UK has recently identified genetically distinct $M$. abscessus from individuals attending a single centre, ${ }^{103}$ many of these isolates fall within international dominant circulating clones and thus arose through transmission. ${ }^{41}$ The mechanism of transmission remains unclear. Epidemiological analysis suggests direct person-to-person spread is unlikely ${ }^{40}$ and indicates that environmental soiling through the generation of long-lived infectious aerosols and contaminated fomites (promoted by the inherent desiccation- and detergent-resistance of $M$. abscessus) may be responsible for transmission. In response to these findings, infection control policies in the USA ${ }^{104}$ and $\mathrm{UK}^{105}$ have been revised, but their impact remains to be determined.

\section{Evidence statements}

Person-to-person transmission may be an important mechanism for the acquisition of M. abscessus, at least within people with CF. (Evidence level $2++$ )

There is no published evidence suggesting person-to-person transmission of other NTM species.

\section{Recommendation}

- Adequate infection control policies need to be implemented in both inpatient and outpatient settings to minimise risks of 
person-to-person transmission of M. abscessus in individuals with CF. (Grade B)

\section{SECTION 5: HOW SHOULD THE LUNG DISEASE ATTRIBUTABLE TO NTM INFECTION BE DEFINED?} Evidence summary

As NTM are ubiquitous organisms that are frequently found in drinking water, the culture of NTM from a respiratory tract culture does not necessarily indicate the presence of lung disease attributable to NTM infection. Thus to determine the clinical relevance of a positive NTM culture, it is essential to distinguish transient or persistent colonisation from infection, the latter indicating tissue invasion and the potential to develop progressive lung disease.

The term NTM-pulmonary disease (NTM-PD) is commonly used to make this distinction and was defined in the ATS/IDSA statement on the Diagnosis, Treatment and Prevention of Nontuberculous Mycobacterial Diseases published in 2007. ${ }^{1}$ Unlike TB where one positive culture is usually sufficient to define infection, to meet the ATS/IDSA criteria for NTM-PD a patient must have characteristic symptoms, compatible radiology, and two or more positive sputum samples of the same NTM species or one positive bronchial wash/lavage or compatible histopathological findings with one positive culture. Other potential causes of pulmonary disease must also be excluded. The microbiological criteria are based on the findings of an observational study from Japan that involved patients with chronic respiratory disease and one or more sputum cultures positive for MAC. ${ }^{106}$ Only $2 \%$ of patients with a single positive culture developed progressive radiological change compared with $98 \%$ of patients with two or more positive cultures. Subsequent studies confirm that the risk of developing NTM-PD in the context of only one NTM positive culture following initial investigation is between $4 \%$ and $14 \%,{ }^{107} 108$ but the risk is likely to be higher in specific patient groups such as CF. ${ }^{109}$ The ATS/IDSA clinical criteria (symptoms and radiology) for NTM-PD are based on clinical experience derived largely from managing patients with MAC, M. abscessus complex and M. kansasii. ${ }^{1}$ While these diagnostic criteria for NTM-PD have not been validated in any patient group or for any specific organism, they have been widely adopted in clinical practice, in research and in clinical trials relating to NTM infection.

\section{Evidence statement}

A single NTM isolate from sputum, which is not isolated again on repeated culture, is usually of no clinical relevance. (Evidence level 3)

Individuals with two or more isolates of the same NTM from repeated sputum cultures are more likely to develop radiological evidence of disease. (Evidence level 3)

\section{Recommendation}

- In the absence of robust evidence to support an alternative definition and due to the clinical and research benefits of having a uniform definition, use of the ATS/IDSA 2007 definition of NTM-pulmonary disease is recommended ${ }^{1}$ (see box 1). (Grade D)

\section{Good practice point}

$\checkmark$ The management of coexisting lung conditions/infections should be optimised before ascribing clinical decline to NTM-pulmonary disease.
Box 1 Clinical and microbiological criteria for diagnosing non-tuberculous mycobacterial lung disease (modified with permission from Griffith et al' $)$

\section{Clinical (both required)}

1. Pulmonary symptoms, nodular or cavitary opacities on chest radiograph, or a high-resolution CT scan that shows multifocal bronchiectasis with multiple small nodules. and

2. Appropriate exclusion of other diagnoses.

\section{Microbiological}

1. Positive culture results from at least two separate expectorated sputum samples; if the results are nondiagnostic, consider repeat sputum AFB smears and cultures. or

2. Positive culture results from at least one bronchial wash or lavage.

or

3. Transbronchial or other lung biopsy with mycobacterial histopathological features (granulomatous inflammation or AFB) and positive culture for NTM or biopsy showing mycobacterial histopathological features (granulomatous inflammation or AFB) and one or more sputum or bronchial washings that are culture-positive for NTM.

Reprinted with permission of the American Thoracic Society.

Copyright @ 2017 American Thoracic Society.

Cite: Griffith DE, Aksamit T, Brown-Elliott BA, et al. An official ATS/IDSA statement: diagnosis, treatment, and prevention of nontuberculous mycobacterial diseases. Am J Respir Crit Care Med 2007;175:367-416.

The American Journal of Respiratory and Critical Care Medicine is an official journal of the American Thoracic Society.

AFB, acid-fast bacilli; NTM, non-tuberculous mycobacteria

\section{SECTION 6: WHAT SAMPLES SHOULD BE USED TO DETECT PULMONARY NTM INFECTION? \\ Evidence summary}

Currently sputum, induced sputum, bronchial washings and bronchoalveolar lavage fluid are used to detect pulmonary NTM infection. There have been no systematic comparisons of these methods of NTM detection. Transbronchial biopsies may reveal NTM (on microscopy or culture) and may demonstrate granulomatous inflammation (supporting NTM disease rather than transient colonisation), although clearly have an associated procedural risk which needs to be considered. ${ }^{110}$ Tracheal aspirates, pleural fluid and tissue samples, and lung biopsies, have all been used to detect NTM infection and may be useful in the appropriate clinical setting. ${ }^{111-113}$ Oropharyngeal swabs can fail to detect NTM infection since they frequently do not obtain sufficient lower respiratory tract material for culture. ${ }^{114}$ There is currently no supporting evidence for the culture of gastric aspirates, faecal specimens or urine to detect NTM infection in HIV-negative patients. Laboratory processing of samples should ideally be performed within 24 hours of collection to avoid bacterial overgrowth, which can reduce NTM viability and prevent successful decontamination. ${ }^{115}$ Studies have shown that refrigeration of samples may improve mycobacterial detection from sputum samples and should be considered if delays longer than 24 hours in processing are anticipated. ${ }^{116}$

Antigen skin testing has been used to detect NTM exposure, ${ }^{117} 118$ but has not been shown to be a valid detection method for NTM infection. Serological testing has been examined in a number of studies as a detection method for NTM 
infection. In one study from Romania, NTM serodiagnosis correlated well with MAC culture results, ${ }^{119}$ while the use of IgA serodiagnosis in Japan was found to have a sensitivity of $84 \%$ and specificity of $100 \%$ for MAC infection. ${ }^{120-122}$ To date these (and other serological tests ${ }^{123}$ ) have not been sufficiently validated in different settings and in different patient cohorts to fully understand their utility and therefore recommend their routine use.

\section{Evidence statement}

Sputum, induced sputum, bronchial washings or bronchoalveolar lavage samples and transbronchial biopsies can be used to evaluate individuals suspected to have NTM-pulmonary disease. (Evidence Level 3)

Delays in sample processing can lead to reduced recovery of NTM from sputum. (Evidence Level 3)

Oropharyngeal swabs can fail to detect NTM pulmonary infection. (Evidence Level 3)

NTM serology has not been sufficiently validated to confirm or exclude NTM-pulmonary disease. (Evidence Level 3)

\section{Recommendations}

- Sputum, induced sputum, bronchial washings, bronchoalveolar lavage or transbronchial biopsy samples can be used to evaluate individuals suspected to have NTM-pulmonary disease. (Grade D)

- Whenever possible, less invasive sampling should be attempted first to minimise procedural risks. (Grade D)

- Respiratory samples should be processed within 24 hours of collection (or refrigerated at $4^{\circ} \mathrm{C}$ if delays are anticipated). (Grade D)

- Oropharyngeal swab culture or serology testing should not be used to diagnose NTM pulmonary infection. (Grade D)

\section{Good practice points}

$\checkmark$ Respiratory specimens should be collected with appropriate infection control precautions, including personal protective equipment, given the frequent differential diagnosis of $M$. tuberculosis infection. ${ }^{2}$

$\checkmark$ If sputum cultures are negative but clinical suspicion of NTM infection is high, consider preforming CT-directed bronchial washings to obtain targeted samples.

$\checkmark$ If individuals undergoing diagnostic evaluation for NTM infection are taking antibiotics that may impair NTM growth (such as aminoglycosides, macrolides, tetracyclines, co-trimoxazole, linezolid), consider discontinuing these antibiotics 2 weeks prior to collecting samples.

\section{SECTION 7: WHAT MICROBIOLOGICAL TESTS SHOULD BE USED TO DETECT NTM IN RESPIRATORY SAMPLES? Evidence summary}

There are a number of methods that can be used to detect NTM from respiratory samples, including microscopy, culture and molecular techniques.

\section{Rapid detection}

Microscopy

Microscopy allows the direct evaluation of bacterial burden and may reveal false-negative culture results caused by excessive sample decontamination or overgrowth of conventional bacteria. However, its sensitivity is greatly influenced by sample quality, mycobacterial species and laboratory expertise, and therefore should not be used as the only indicator of bacterial load. ${ }^{124-127}$ Microscopy cannot reliably distinguish NTM from M. tuberculosis. It should be noted that the limited available data on $M$. abscessus suggest that, in contrast to TB where smear-positive people cause most transmission, smear-negative individuals may still contribute to cross-infection ${ }^{40}$ and should be considered potentially infectious to susceptible contacts. ${ }^{105}$ Nevertheless, microscopy provides a useful adjunct to culture and should be routinely carried out using auramine-phenol staining rather than the Ziehl-Neelsen method due to enhanced sensitivity. ${ }^{124-127}$

\section{Direct molecular detection}

Although molecular techniques to detect NTM in respiratory specimens are now commercially available, they are costly and remain less sensitive than conventional acid-fast bacilli (AFB) culture. Examples of methods available for direct NTM detection include PCR restriction analysis, sputum rpoB gene analysis ${ }^{128}$; and the GenoType Mycobacteria Direct test. ${ }^{129}{ }^{130}$ To date, clinical validation of these tests in different patient cohorts and for different NTM species is incomplete and therefore their role in routine clinical practice remains to be determined. However one very useful application of direct molecular detection is to rapidly differentiate $M$. tuberculosis complex from NTM in smear-positive samples. ${ }^{131}$

\section{Mycobacterial culture}

The most sensitive method to detect NTM from respiratory samples is by culture. Although guidelines for NTM culture have been currently published in both the UK and USA, the optimal methods for decontamination, type of media, duration and temperature of cultures have yet to be fully determined. Optimal results may require the use of multiple media types and incubation temperature.

Prior to inoculation for mycobacterial culture, decontamination of non-sterile specimens contaminated with commensal flora is required to prevent overgrowth of mycobacterial cultures. A number of protocols may be used: $\mathrm{N}$-acetyl L-cysteine (Nalc)-sodium hydroxide $(\mathrm{NaOH}) 2 \%$, oxalic acid and Nalc- $\mathrm{NaOH} 1 \%$. Since enhanced decontamination protocols adversely impact on NTM viability in samples ${ }^{132}$ ideally for NTM isolation, a two-step approach to sample processing should be adopted. ${ }^{133}$ Virtually all European clinical microbiology laboratories currently use an $\mathrm{Nalc}-\mathrm{NaOH}$ decontamination step prior to mycobacterial culture. ${ }^{124} 133134$ The use of a second decontamination step using oxalic acid has been shown to permit the recovery of NTM from persistently contaminated samples, although with reduced sensitivity. ${ }^{135} 136$

There are two main methods of culture in general use: automated liquid culture systems (usually Mycobacteria Growth Indicator Tube (MGIT); BD Biosciences, Sparks, Maryland, USA), which are more rapid and sensitive than solid media (usually Löwenstein-Jensen or Middlebrook 7H10 and 7H11 media), although more recently agar designed for Burkholderia cepacia complex culture has been successfully used. ${ }^{137}$ RGM ('rapid growing mycobacteria') media has compared favourably with MGIT liquid culture for the detection of NTM. ${ }^{138} 139$ The major advantages of solid culture are that colony morphology can be evaluated, mixed infections can be identified and NTM can be detected despite incomplete sample decontamination. Combination of liquid and solid media gives rise to the greatest recovery rates: MGIT, Bactec 460 and solid media recovered 85\%,72\% and $62 \%$ of MAC, respectively, ${ }^{140}$ while some mycobacteria will grow in one but not another system. 
Culture of NTM can be difficult and may not be routinely optimised even in mycobacterial reference laboratories. Routinely solid and liquid culture is performed at $35^{\circ} \mathrm{C}$ (the optimal recovery temperature for $M$. tuberculosis), but many NTM species have optimum growth at $28^{\circ} \mathrm{C}-30^{\circ} \mathrm{C}$ (such as M. abscessus) or $45^{\circ} \mathrm{C}$ (such as M. xenopi). ${ }^{1}$ Some NTM species require supplemented media (such as $M$. genavense and M. haemophilum) or extended duration of incubation (such as M. malmoense). Indeed some NTM species have yet to be successfully cultured (such as M. tilburgii ${ }^{141}$ ). The duration of both liquid and solid culture methods has not been extensively tested, but the vast majority of pathogenic NTM will grow by 6 weeks. The current UK recommendation is to culture for 8 weeks extending to 12 weeks if necessary. ${ }^{142}$

\section{Evidence statements}

Auramine-phenol staining on liquefied and concentrated specimens is the method of choice for microscopic detection of mycobacteria as this is more sensitive and quicker than other available methods. (Evidence level $2++$ )

Microscopy alone cannot reliably differentiate NTM from $M$. tuberculosis complex. (Evidence level $2++$ )

Decontamination of non-sterile specimens is required prior to culture for mycobacteria to prevent bacterial overgrowth. The best method for decontamination of respiratory specimens varies with the clinical and epidemiological context. (Evidence level 2-)

Sample culture on both solid and liquid media is the most sensitive detection method currently available for NTM from respiratory samples. (Evidence level 2+)

\section{Recommendations}

- A validated rapid method should be used to detect NTM in respiratory samples. (Grade D)

- All respiratory samples should be stained using auraminephenol after liquefaction and concentration and then examined by microscopy. (Grade B)

- Respiratory tract samples should be cultured (following decontamination) on solid and liquid media in a ISO15189-accredited clinical laboratory for 8 weeks extending to 12 if necessary. (Grade D)

- Routine use of non-culture-based detection methods is not recommended at the present time. (Grade D)

\section{Good practice point}

$\checkmark \quad$ If there is high clinical suspicion of NTM infection but negative sample cultures, consider discussing with a mycobacterial reference laboratory about (1) the possibility of culture on alternative media, at different temperatures, and/or for extended durations or (2) the utility of molecular detection methods.

\section{SECTION 8: WHAT MICROBIOLOGICAL TESTS SHOULD BE USED TO SPECIATE AND TYPE NTM FROM RESPIRATORY SAMPLES?}

\section{Evidence summary}

Correct species identification of NTM isolates is clinically important since NTM species differ in their potential to cause clinical disease in humans ${ }^{143}$ and in their response to specific antibiotics. In the case of M. abscessus, subspeciation into M. $a$. abscessus, $M$. a. bolletii and M. a. massiliense may predict treatment response ${ }^{144}$ and potentially permit targeted therapy. ${ }^{145} M$. a. massiliense harbours a partial erm 41 gene deletion preventing inducible macrolide resistance ${ }^{144146}$ and leads to more successful outcomes with macrolide-based antibiotic regimens than in infections with M. a. abscessus (which has a full length, functional erm 41 gene). ${ }^{144}$

Over recent years clinical laboratory methods of speciation have moved from biochemical to molecular techniques. Matrix-assisted laser desorption ionisation-time of flight (MALDI-TOF) mass spectrometry has shown considerable promise in providing rapid speciation of NTM. ${ }^{147-151}$ However, the optimal method for protein extraction from mycobacteria and the exact discriminatory power of this method have yet to be fully established. In a recent report of M. abscessus subtyping compared with multilocus sequence typing (MLST), MALDI-TOF showed good discrimination in $94 \%$ of isolates. $^{152153}$

Of the molecular techniques available, line probe assays (eg, Hain CM and AS) are in widespread use in the UK allowing reasonable discrimination and identification of common species of NTM, more recently, subspeciation and selected antibiotic resistance of M. abscessus. ${ }^{154-157}$ An alternative method, PCR product restriction analysis, in which amplified gene fragments are restriction-digested to yield different-sized fragments which are then resolved by gel electrophoresis and correlate with specific species, ${ }^{158}$ is mostly used in low-resource settings and is at least comparable to the line probe assays. ${ }^{159}$ The final technique is (partial) gene sequencing, which permits a higher level of discrimination, often to subspecies level, but is only available in laboratories with access to sequencing facilities. The choice of the optimal sequencing strategy is not straightforward. Although partial 16S rRNA gene sequencing provides insufficient discrimination, particularly between $M$. abscessus and $M$. chelonae, ${ }^{160}$ a number of other gene sequences (such as partial $h s p 65$ and $r p o B$ gene sequences) have been successfully used. ${ }^{161-163}$ For subspeciation of M. abscessus, an MLST approach has recently been validated. ${ }^{162} 164165$ An alternative strategy to subspeciation is to measure erm gene-associated inducible macrolide resistance by phenotypic DST. This does not distinguish accurately between different $M$. abscessus subspecies but does determine the key factor of whether or not there is inducible macrolide resistance.

\section{Molecular typing}

The exact role for molecular typing in NTM infection is currently unclear. Given recent reports of likely person-to-person transmission of $M$. abscessus between individuals with CF and, in one case, non-CF bronchiectasis, ${ }^{40} 41$ it is becoming evident that molecular typing of M. abscessus may have important roles in epidemiology, postoutbreak surveillance and in validation of new infection control measures. ${ }^{105}$ The optimal method is also in evolution with $65 \mathrm{kDa}$ heat shock protein analysis, multilocus sequence typing, MALDI-TOF and next-generation sequencing all likely to have roles. However, in light of the slow mutation rates of $M$. abscessus, only whole genome sequencing can provide the molecular discrimination to definitively identify person-to-person transmission. ${ }^{4041}$

\section{Evidence statements}

Different NTM species have varying potential to cause clinical disease in humans. (Evidence level $2++$ )

MALDI-TOF mass spectrometry is more discriminatory than Hain CM/AS for distinguishing between NTM and between M. abscessus subspecies. (Evidence level $2++$ )

Detection of person-to-person transmission of M. abscessus requires whole genome sequencing. (Evidence level 3) 


\section{Recommendations}

- All NTM isolates from respiratory samples should be identified to at least species level using validated molecular or mass spectrometry techniques. (Grade B)

- Isolates of M. abscessus should be subspeciated using appropriate molecular techniques. (Grade C)

- If person-to-person transmission of M. abscessus is suspected, isolates should be typed, preferably using whole genome sequencing (Grade $\mathrm{C}$ ).

\section{SECTION 9: DOES IN VITRO DST PREDICT RESPONSE TO ANTIBIOTIC TREATMENT IN PEOPLE WITH NTM PULMONARY INFECTION? \\ Evidence summary}

The clinical value of in vitro DST in the treatment of infection requires consistent correlation between in vitro susceptibility to a drug by a defined method to clinically achievable drug concentrations at the site of infection and good outcome to treatment with that drug. The value of DST for pulmonary NTM infection is mostly uncertain.

In considering how to treat NTM infections with combinations of antimicrobials, factors such as antimicrobial synergy, ${ }^{166-168}$ indifference and antagonism should be considered, as well as other potential drug interactions and toxicities. The enthusiasm to 'do something' and the perception that 'more is better' should be tempered by the need to avoid harm. In North America insurance companies require NTM sensitivity testing to be carried out to guide therapy. This may have the benefit of encouraging research into this field, but also may encourage NTM sensitivity testing where the evidence for its utility is limited. Although the European Committee on Antimicrobial Susceptibility Testing has presently no guidelines for DST of NTM, ${ }^{114}$ the US CLSI guidelines recommend testing using broth microdilution and provide breakpoint concentrations to interpret minimum inhibitory concentrations (MICs) as 'susceptible' or 'resistant'. ${ }^{169}$ However these cut-offs have had very limited clinical validation. Moreover, whereas limited pharmacokinetic data are now available for MAC lung disease to support breakpoint concentrations, ${ }^{170}$ there are no representative pharmacokinetic or pharmacodynamic data to guide treatment of other NTM infections.

\section{Slow-growing mycobacteria}

Breakpoints for clarithromycin susceptibility of MAC have been validated in HIV-related disseminated MAC disease and in retrospective series of MAC lung disease. ${ }^{171-173}$ Since the presence of macrolide resistance predicts worse clinical outcomes ${ }^{174-176}$ and requires augmented treatment, ${ }^{175}$ susceptibility to macrolides should be tested on isolates prior to treatment initiation and during treatment in refractory cases such as those individuals who (1) fail to culture-convert after 6-12 months of NTM treatment; (2) reculture MAC after initial culture conversion while on NTM treatment; or (3) reculture MAC after completion of NTM treatment.

A recent study has shown that amikacin MICs $>64 \mathrm{mg} / \mathrm{L}$ are found only in MAC isolates that have mutations associated with amikacin resistance, that is, in the $16 \mathrm{~S}$ rRNA gene. These strains are cultured from patients with significant aminoglycoside exposure, such as patients with $\mathrm{CF}$, and for disease caused by these strains, amikacin is unlikely to have any beneficial effect. ${ }^{177}$

Rifampicin, ethambutol and clofazimine susceptibility results did not predict outcome of monotherapy for MAC infection in HIV-positive individuals. ${ }^{172} 178$
For M. kansasii infection rifampicin 'resistance' correlates best with treatment failure ${ }^{14}$ and 'resistance' to isoniazid or ethambutol may occur, often associated with rifampicin resistance. ${ }^{179} 180$ For rifampicin-resistant infections further testing may be recommended, ${ }^{14} 169$ but interpretation of results should be made with caution.

For M. simiae, the role of DST is unknown, although the generally poor outcomes of treatment have been correlated with a lack of synergistic activity between rifampicin and ethambutol, an in vitro observation that still awaits clinical validation. ${ }^{181}$

\section{Rapid-growing mycobacteria}

For rapidly-growing mycobacteria including $M$. abscessus, clinical validation has only been performed in series of extrapulmonary disease $\mathrm{e}^{182}$ and only for cefoxitin, aminoglycosides and co-trimoxazole. In series of M. abscessus lung disease, the outcomes of macrolide-based treatment are generally poor and do not correlate well with in vitro susceptibilities, ${ }^{171} 183$ potentially due to $\mathrm{erm}(41)$-dependent inducible macrolide resistance and relative short duration of adequate regimens, which were often interrupted because of toxicity. Indeed, in the absence of a functional erm(41) gene, response to macrolide-containing treatments has been good. ${ }^{144}$ The CLSI has recommended routine testing for inducible macrolide resistance by performing extended incubation of isolates in the presence of clarithromycin as inducible resistance may predict treatment failure. ${ }^{169}$ Some molecular methods to assess drug susceptibility exist, but are not yet routinely available. For example, sequencing of the $16 \mathrm{~S}$ rRNA and $23 \mathrm{~S}$ rRNA genes can reveal mutations associated with high-level resistance to aminoglycosides and macrolides, respectively, ${ }^{171} 177$ in M. abscessus and other NTM species.

\section{Evidence statements}

In vitro resistance to macrolides and amikacin is associated with treatment failure in MAC infection. (Evidence level $2++$ )

In vitro resistance to rifampicin is associated with treatment failure in $M$. kansasii infection. (Evidence level $2++$ )

In vitro resistance to macrolides is associated with treatment failure in M. abscessus pulmonary infection. (Evidence level $2++)$

In vitro resistance to cefoxitin, amikacin and co-trimoxazole is associated with treatment failure in extrapulmonary M. abscessus infection. (Evidence level 2++)

\section{Recommendations}

- Drug susceptibility testing and reporting should follow the CLSI guidelines. (Grade D)

- For MAC, clarithromycin and amikacin susceptibility testing should be performed on an isolate taken prior to initiation of treatment and on subsequent isolates if the patient fails to respond to treatment or recultures MAC after culture conversion (Grade C).

- Macrolide-resistant MAC isolates should be tested against a wider panel of antibiotics to guide, but not dictate, treatment regimens. (Grade D).

- For M. kansasii, rifampicin susceptibility testing should be performed on an isolate prior to initiation of treatment and on subsequent isolates if the patient fails to respond to treatment or recultures $M$. kansasii after culture conversion. (Grade D)

- Rifampicin-resistant M. kansasii isolates should be tested against a wider panel of antibiotics to guide, but not dictate, treatment regimens. (Grade D) 
- Susceptibility testing for M. abscessus should include at least clarithromycin, cefoxitin and amikacin (and preferably also tigecycline, imipenem, minocycline, doxycycline, moxifloxacin, linezolid, co-trimoxazole and clofazimine if a validated method is available) to guide, but not dictate, treatment regimens. (Grade D)

\section{Good practice points}

$\checkmark$ Susceptibility testing should only be carried out on isolates where there is clinical suspicion of disease (to avoid unnecessary cost and conserve laboratory resources).

$\checkmark$ Reporting of MIC and critical concentration rather than susceptible or resistant may be more appropriate in the belief that a drug that has a very high MIC is unlikely to be active in vivo, whereas one just above a putative 'critical concentration' may have some activity, especially if combined with additive or synergistic agents.

\section{SECTION 10: WHAT INVESTIGATIONS SHOULD BE PERFORMED IN INDIVIDUALS SUSPECTED OF HAVING NTM- PULMONARY DISEASE?}

\section{Evidence summary}

In individuals with compatible symptoms, the ATS/IDSA microbiological and radiological criteria must be met before the diagnosis of NTM-PD is made. ${ }^{1}$ While there are no prospective controlled studies indicating which microbiological and radiological investigations should be performed or how they should be interpreted, several case series and other studies provide some insights (figure 1).

\section{Respiratory tract cultures \\ Sputum}

In a retrospective observational study of Japanese patients with MAC, $98 \%$ of patients with two or more positive sputum cultures went on to develop chest X-ray changes indicative of progressive disease. ${ }^{106}$ In contrast, just 2 of 114 patients from whom only a single positive sputum culture was obtained developed progressive radiological change during follow-up of at least 12 months.

In a retrospective study of patients with CF in the USA and at least one positive NTM culture, $>60 \%$ of subjects had transient or persistent infection and did not meet the ATS/IDSA definition of NTM-PD. ${ }^{109}$

In a study from Taiwan of patients with a single isolate of MAC, M. chelonaelabscessus complex, M. kansasii or M. fortuitum from three or more sputum samples collected within 1 month and who had at least 1 year of follow-up data, 44/202 (22\%) had subsequent cultures of the same NTM species, but only 8/202 (4\%) developed NTM-PD. ${ }^{107}$

In a study from South Korea, involving 190 patients with an initial single positive sputum culture of either MAC, M. abscessus

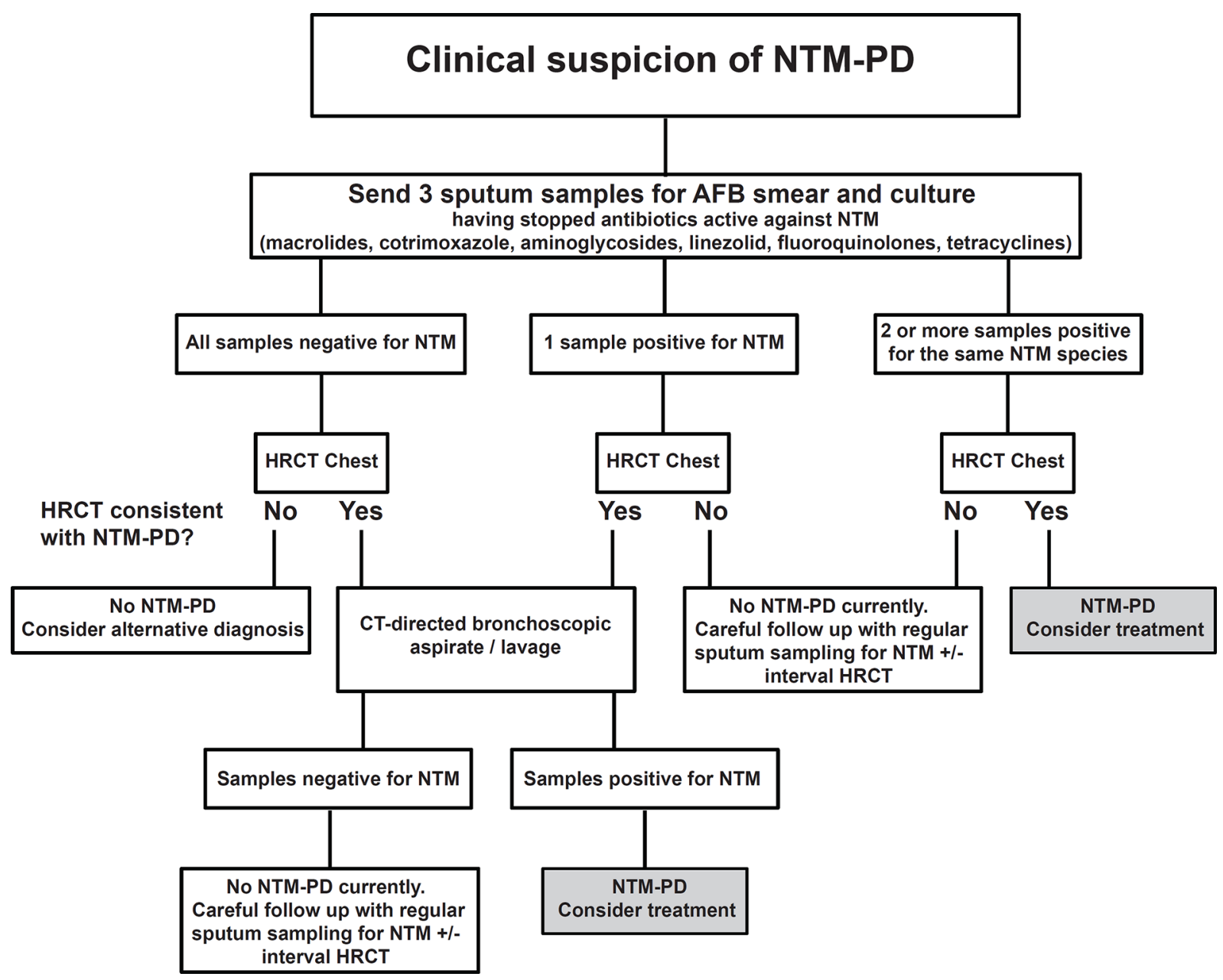

Figure 1 An algorithm for the investigation of individuals with clinical suspicion of NTM-pulmonary disease (AFB, acid-fast bacilli; HRCT, highresolution CT; NTM-PD, non-tuberculous mycobacterial pulmonary disease). Modified from Thorax with permission (Floto et al). ${ }^{336}$ 
complex or M. kansasii, 26 patients (14\%) had an additional positive culture of the same NTM species and met the ATS/IDSA criteria for NTM-PD a median of 30 (IQR 15-46) months after the first positive sputum culture. ${ }^{108}$

NTM can be isolated from induced sputum samples, ${ }^{184} 185$ but there are no data comparing the yield of NTM from spontaneously expectorated versus induced sputum samples.

\section{Evidence statements}

A single NTM isolate from sputum, which is not isolated again on repeated culture, is usually of no clinical relevance. (Evidence level 3)

Individuals with two or more isolates of the same NTM from repeated sputum cultures are more likely to develop radiological evidence of disease. (Evidence level 3)

\section{Recommendation}

- A minimum of two sputum samples collected on separate days should be sent for mycobacterial culture when investigating an individual suspected of having NTM-pulmonary disease. (Grade D)

\section{Bronchoscopy}

In a prospective study evaluating the role of sputum culture, bronchial washings and transbronchial lung biopsy in 26 patients (4 male and 22 female) with a mean age of 62 years and CT changes compatible with NTM-PD (bronchiectasis, tree-in-bud opacity and nodules), 15/26 patients cultured NTM, 8 (53\%) of whom only cultured NTM from the bronchial wash. ${ }^{111}$ The bronchial wash sample was culture-positive for NTM in all patients in whom transbronchial biopsy yielded granulomas. Similar findings were also reported in a number of retrospective case series. $^{112186187}$

\section{Evidence statements}

CT-directed bronchial washings are more sensitive than spontaneously expectorated sputum samples for isolating NTM in individuals with nodular bronchiectatic disease. (Evidence level 3)

Bronchial washings can be used to identify NTM in individuals who are unable to expectorate sputum. (Evidence level 3)

Bronchial washings frequently culture NTM in individuals with histological evidence of granulomatous inflammation on transbronchial biopsy. (Evidence level 3)

\section{Recommendations}

- Individuals suspected of having NTM-pulmonary disease whose sputum samples are consistently culture-negative for mycobacteria should have CT-directed bronchial washings sent for mycobacterial culture. (Grade D)

- Individuals suspected of having NTM-pulmonary disease who are unable to expectorate sputum should have CT-directed bronchial washings sent for mycobacterial culture. (Grade D)

- Transbronchial biopsies should not be performed routinely in individuals suspected of having NTM-pulmonary disease. (Grade D)

\section{Good practice points}

$\checkmark$ Sputum induction resulting in a positive culture may avoid the need for CT-directed bronchial washings in individuals who are unable to spontaneously expectorate sputum. $\checkmark$ Sputum induction should be considered in individuals suspected of having NTM-pulmonary disease who are unable to spontaneously expectorate sputum and in whom CT-directed bronchial washings are considered inappropriate.

\section{Radiology \\ Chest X-ray}

In a retrospective analysis, 258 of 271 (95\%) patients with M. kansasii had cavitary disease on chest X-ray, and only one patient converted to sputum culture-negative after 2 weeks of treatment. ${ }^{188}$ In the same study, 173 of $226(77 \%)$ patients with MAC had cavitary disease on chest X-ray, of whom 110 remained culture-positive after 2 weeks of treatment. Of the 66 patients with either M. kansasii or MAC who did not have cavities on chest X-ray, 43 (65\%) became sputum culture-negative after 2 weeks of antimycobacterial chemotherapy or treatment with airway clearance.

In a prospective non-comparative trial of thrice-weekly antibiotic treatment for MAC-pulmonary disease, having non-cavitary compared with cavitary disease was associated with a fourfold increase in culture response. ${ }^{189}$

In a retrospective study of 180 patients receiving standard therapy for nodular/bronchiectatic MAC lung disease, change in semiquantitative culture results from baseline to month 2 of treatment was significantly associated with improvements in radiological scores during the same time period. ${ }^{185}$

\section{Evidence statement}

In individuals with positive cultures for M. kansasii or MAC, chest X-ray evidence of lung cavitation is indicative of disease and is associated with worse outcomes following treatment. (Evidence level 3)

In individuals receiving treatment for nodular bronchiectatic MAC-pulmonary disease, improvements in radiological appearances are indicative of a positive microbiological response to treatment. (Evidence level 3)

\section{Recommendation}

- A chest X-ray should be performed in individuals suspected of having NTM-pulmonary disease. (Grade D)

\section{Computed tomography}

Features commonly identified by CT in patients with NTM-PD include bronchiectasis (particularly affecting the middle lobe and/or lingula), centrilobular nodules, tree-in-bud opacities, consolidation, cavitation and pre-existing lung disease such as emphysema. ${ }^{190-198}$ While these changes are associated with NTM infection and often indicate the need for further investigation, they are not specific for NTM infection ${ }^{111}$ and cannot distinguish between different species of NTM or between TB and NTM. ${ }^{188} 190199$

Patterns of disease seen on CT correlate with response to treatment and prognosis. In a prospective non-comparative trial of antibiotic treatment for MAC-pulmonary disease, patients with non-cavitary disease on CT showed more favourable early responses to treatment compared with patients with cavitary disease. ${ }^{189}$ These findings are supported by case series in which patients with cavitary disease and/or consolidation had worse outcomes than those with predominantly nodular/bronchiectatic changes without cavitation. ${ }^{200-204}$ However, those with cavitary disease may be more likely to have other factors associated with an unfavourable prognosis such as a history of COPD or TB, 
heavier tobacco use, sputum smear positivity, more dyspnoea and chronic pulmonary aspergillosis. ${ }^{205}$

A subgroup of mainly middle-aged/elderly female patients with no history of pre-existing lung disease appear predisposed to developing MAC (and possibly other NTM) infection centred mainly, but not exclusively, in the middle lobe and/ or lingula. ${ }^{62} 187193$ The chest X-ray may be relatively normal, but the CT can reveal clusters of small nodules, tree-in-bud opacification and bronchiectasis. Sputum expectoration is characteristically minimal or absent and diagnostic confirmation of NTM infection may require bronchoscopy and bronchial washings.

\section{Evidence statements}

CT features of NTM-pulmonary disease include centrilobular nodules, tree-in-bud opacity, bronchiectasis (particularly affecting the middle lobe and/or lingula), consolidation and cavitation. (Evidence level 3)

Abnormalities seen on CT cannot reliably distinguish between infections caused by TB and NTM, or between infections caused by different species of NTM. (Evidence level 3)

Individuals with CT evidence of cavitary disease and/or consolidation have a worse prognosis and a poorer response to antimycobacterial treatment than those showing predominantly nodular/bronchiectatic changes without cavitation. (Evidence level 3)

MAC-pulmonary disease in white postmenopausal women without a history of pre-existing lung disease is frequently associated with CT evidence of bronchiectasis, nodules and tree-in-bud opacity in the middle lobe and lingula. (Evidence level 3)

\section{Recommendation}

- A CT scan should be performed in individuals suspected of having NTM-pulmonary disease. (Grade D)

\section{OTHER INVESTIGATIONS \\ Serology}

Several studies have evaluated the role of serology in the diagnosis of MAC-pulmonary disease by measuring serum IgA antibody against glycopeptidolipid core antigen, a cell surface antigen that is found in MAC, but not M. tuberculosis or M. kansasii. A multicentre study in Japan showed the ELISA had a sensitivity of $84 \%$ and specificity of $100 \%,{ }^{121}$ and a study in Korea reported a sensitivity of $85 \%$ and specificity of $100 \% .^{206}$ However, a study performed in the USA reported a sensitivity of $52 \%$ and a specificity of $94 \%,{ }^{207}$ and similar results were reported in a study performed in Taiwan. ${ }^{208}$ In addition to the low sensitivity in some settings, the clinical utility of the assay might also be affected by cross-reactivity with some rapidly growing mycobacteria including M. abscessus complex. ${ }^{206}$

An assay measuring serum antibody against the mycobacterial antigen A60 found it to be both sensitive (87\%) and specific (95\%) for the diagnosis of $M$. abscessus complex infection in people with CF, with IgG titres being sixfold higher in 15 patients with M. abscessus complex compared with 144 NTM-negative controls. ${ }^{123}$ The authors suggested that the assay had clinical utility in (1) supporting initiation of microbiological investigation when there is a suspicion of M. abscessus complex-PD, (2) to assess disease activity of documented $M$. abscessus complex-PD and (3) to monitor disease activity while on treatment for M. abscessus complex-PD. Similar findings were reported in a more recent CF study, in which patients with M. abscessus complex-PD had median anti-M. abscessus complex IgG levels sixfold higher than patients with no history of infection, and the sensitivity and specificity of the test were $95 \%(95 \%$ CI $74 \%$ to $99 \%)$ and $73 \%(95 \%$ CI $67 \%$ to $78 \%)$, respectively. $^{209}$

\section{Skin testing and interferon gamma release assays}

Neither purified protein derivative (PPD) tuberculin skin testing nor interferon gamma release assays can reliably distinguish between infection caused by TB and MAC. ${ }^{210} 211$ Skin testing using PPD-B is relatively sensitive, but has poor specificity for NTM and its use in practice is limited by problems with standardisation and bioequivalence of antigens. ${ }^{210}$

\section{Positron emission tomography}

Positron emission tomography was used to assess disease activity and therapeutic response by comparing pretreatment and post-treatment F-FDG uptake in peripheral lung nodules caused by MAC $(n=22)$ and TB $(n=25) .{ }^{212}$ F-FDG uptake significantly decreased during or following treatment in all 14 patients with follow-up scans.

\section{Investigations for underlying immunodeficiencies}

Although frequently presenting with disseminated NTM infection, immunodeficiencies should also be considered in individuals with recurrent, persistent or severe pulmonary infection, particularly with NTM species that are normally non-pathogenic, as well as in those presenting with NTM disease in the absence of structural lung disease, or in those presenting at a young age. In these cases referral to a clinical immunologist is recommended.

\section{Evidence statement}

Serological testing has clinical utility in the diagnosis of NTM-pulmonary disease and in monitoring response to treatment in some populations, but this is not a consistent finding. (Evidence level 3)

Positron emission scanning, skin testing and interferon gamma release assays are not sufficiently sensitive and/or specific for use in evaluating individuals suspected of having NTM-pulmonary disease. (Evidence level 3)

\section{Recommendations}

- There is insufficient evidence to recommend the routine use of serological testing for diagnosis and monitoring of individuals with NTM-pulmonary disease. (Grade D)

- Positron emission scanning, skin testing and interferon gamma release assays should not be used in the evaluation of individuals suspected of having NTM-pulmonary disease. (Grade D)

\section{SECTION 11: WHAT FACTORS INFLUENCE WHEN NTM TREATMENT SHOULD BE STARTED? \\ Evidence summary}

There are no randomised controlled trial data that address when NTM treatment should be started. However, several case series have identified clinical characteristics that are associated with progressive NTM disease.

\section{Microbiology}

Clinical outcomes differ between mycobacterial species. In patients from Denmark, M. xenopi was associated with a worse prognosis than MAC after controlling for comorbidity, ${ }^{28}$ and in the BTS-sponsored randomised controlled treatment trials 
patients with M. xenopi had a higher mortality than those with MAC or M. malmoense. ${ }^{213} 214$ Within the Netherlands, there were marked differences in clinical relevance between NTM species, with M. malmoense, M. xenopi, M. szulgai, M. kansasii, $M$. celatum and $M$. genavense being more frequently associated with ATS/IDSA-defined NTM-PD than other species. ${ }^{15}$ In France, patients with CF and positive cultures for M. abscessus complex or MAC were more likely to fulfil ATS/IDSA criteria for NTM-PD than patients with other NTM species. ${ }^{215}$ Furthermore, the clinical relevance of individual species can vary geographically, both within countries and internationally. ${ }^{15}$

Subspecies within the same complex can be associated with different microbiological and clinical outcomes following treatment. For example, within the M. abscessus complex patients with M. massiliense tend to have better treatment responses than those with M. abscessus, ${ }^{76} 144216217$ and within the MAC, clinical relapse/reinfection is more common with M. avium and $M$. chimaera than with $M$. intracellulare in the USA, ${ }^{218}$ whereas in Korea disease due to M. avium was associated with a worse prognosis than that attributable to $M$. intracellulare. ${ }^{219}$ Outcomes may also vary according to mycobacterial genotype. .20-222 $^{22}$

Clinical outcomes differ according to the number of positive NTM cultures and mycobacterial burden. An observational study from Japan showed that the number of MAC-positive cultures was related to the likelihood of radiographical progression, with only $2 \%$ of patients with a single positive culture developing progressive radiological change compared with $98 \%$ of patients with two or more positive cultures. ${ }^{106}$ Patients with smear-positive disease are also more likely to have a poor outcome 220 and less likely to spontaneously culture-convert. ${ }^{223}$ Similarly, the number of mycobacterial colonies correlates with disease progression in untreated patients with MAC. ${ }^{200}$

\section{Clinical characteristics}

Clinical characteristics that appear to confer a worse prognosis have been reported in many retrospective studies. The largest involving 634 patients with MAC $^{201}$ demonstrated male gender, age, comorbidity, non-nodular bronchiectatic features, BMI $<18.5$, anaemia, low albumin and ESR $>50$ to be associated with all-cause mortality. ${ }^{201}$ Similar findings relating to low BMI, ${ }^{197} 220$ cavitatory disease ${ }^{189} 197198202$ and aspergillus coinfection ${ }^{198}$ were reported in smaller studies involving patients with NTM-PD.

\section{Serology}

A variety of biomarkers have been evaluated as diagnostic tests for NTM-PD and as measures of disease activity. For example, IgA against the glycopeptidolipid core antigen showed higher values in patients with MAC-pulmonary disease than controls ${ }^{224}$ and correlated with radiographical changes, ${ }^{225}$ and anti-A60 IgG antibodies were higher in patients with CF with M. abscessus complex that fulfilled the ATS microbiological criteria for disease compared with those that did not. ${ }^{123}$ However, none of the serological data are sufficiently robust to support widespread implementation into clinical practice at the present time.

\section{Summary}

The decision to start treatment is related to, but is distinct from, the ATS/IDSA definition of NTM-PD. ${ }^{1}$ Patients in whom NTM treatment is required will in almost all cases fulfil the ATS/IDSA criteria for NTM-PD, but fulfilling the criteria does not necessarily imply that treatment should be started, as patients can remain stable without antibiotic treatment. ${ }^{201}$
The identification of progressive pulmonary disease attributable to NTM provides a stronger case for treatment. Microbiological features indicating disease progression include an increased frequency of positive versus negative cultures, the development of smear positivity and reduced time to MGIT positivity. CT features indicating disease progression include increased size and/or number of pulmonary nodules, new or worsening lung cavitation, new foci of consolidation/tree-in-bud opacity and worsening extent and/or severity of bronchiectasis. Thus, careful longitudinal assessment may be helpful in determining an individual patient's treatment requirements.

In practice, the decision to offer NTM treatment is based on the amalgamation of patient-related and mycobacterial factors. Patient-related factors include the severity and rate of progression of symptoms and radiological change, the underlying lung disease, the presence of comorbidity, current treatment requirements (eg, immunosuppressive agents such as TNF- $\alpha$ inhibitors) and likely future treatment requirements (eg, lung transplantation). Mycobacterial factors include the pathogenicity of the individual species and the bacterial load. The aim of treatment will also influence when to start treatment. For example, achieving NTM eradication or slowing disease progression may require earlier intervention than treatment directed at symptom control alone.

\section{Evidence statements}

The following patient-related factors are frequently associated with progressive NTM-pulmonary disease: severe symptoms, low BMI, lung cavitation and comorbidity (Evidence level 3).

The following mycobacterial factors are frequently associated with progressive NTM-pulmonary disease: smear positivity, two or more positive mycobacterial cultures of the same organism and particular mycobacterial species (Evidence level 3).

\section{Recommendations}

- The decision to start treatment should be influenced by the severity of NTM-pulmonary disease, the risk of progressive NTM-pulmonary disease, the presence of comorbidity and the goals of treatment. (Grade D)

- Individuals may require a period of longitudinal assessment (symptoms, radiological change and mycobacterial culture results) to inform NTM treatment decisions. (Grade D)

\section{Good practice point}

$\checkmark$ The views of the affected individual should be sought on the potential risks and benefits of starting NTM treatment versus observation (ie, longitudinal assessment of symptoms, radiological change and mycobacterial culture results).

\section{SECTION 12: WHAT ANTIBIOTIC REGIMEN SHOULD BE USED TO TREAT NTM-PD?}

Treatment recommendations are presented for the NTM species that most commonly fulfil the ATS/IDSA microbiologic criteria for NTM-PD within the UK, namely MAC, M. kansasii, M. malmoense, M. xenopi and M. abscessus complex. ${ }^{226}$

\section{SECTION 12A: WHAT ANTIBIOTIC REGIMEN SHOULD BE USED TO TREAT MAC-PULMONARY DISEASE? \\ Evidence summary}

Five randomised controlled trials and several non-comparator studies involving individuals (not known to be HIV-positive) with MAC were identified in the literature search. 
BTS sponsored two randomised controlled treatment trials involving patients with M. malmoense, M. xenopi and MAC. ${ }^{213} 214$ Patients were treated for 2 years and then followed up for a further 3-year period. The first trial included 75 patients with MAC and those randomised to rifampicin, ethambutol and isoniazid had a failure/relapse rate of $16 \%$ compared with $41 \%$ of patients randomised to rifampicin and ethambutol $(\mathrm{p}=0.033)$. There was no difference in mortality. ${ }^{213}$ The second trial randomised 170 patients with MAC to receive either ciprofloxacin or clarithromycin, in combination with rifampicin and ethambutol. ${ }^{214}$ Some patients were also randomised to receive either M. vaccae or no immunotherapy, but no differences in outcomes were found. The results of the antibiotic trial showed that $13 \%$ of patients in the clarithromycin group failed treatment/relapsed compared with $23 \%$ in the ciprofloxacin group, but the all-cause mortality was higher in clarithromycin-treated patients (48\% vs 30\%). The clarithromycin-containing regimen was better tolerated than the ciprofloxacin-containing regimen $(p=0.05)$. A comparison of all treatments evaluated in the two BTS-sponsored RCTs was attempted by the authors, but limited by the different study designs, the small number of participants, the lack of statistical analysis for some outcomes and complex endpoints. $^{214}$

In a multicentre RCT involving $146 \mathrm{HIV}$-negative patients with MAC-pulmonary disease, intramuscular streptomycin $(15 \mathrm{mg} / \mathrm{kg})$ administered three times weekly for the first 3 months of therapy improved sputum conversion rates at the end of 2 years of treatment compared with placebo $(71.2 \%$ vs $50.7 \%$, $\mathrm{p}<0.05$ ), with rifampicin, ethambutol and clarithromycin as the companion drugs. ${ }^{227}$ However, there was no difference in symptoms, radiological response or adverse events, and a third of patients in each group experienced sputum relapse. Four open studies supported a benefit of including streptomycin at the start of treatment. ${ }^{173} 228-230$

The fourth RCT involved 27 patients and demonstrated no difference between gatifloxacin and clarithromycin as companion drugs to rifampicin and ethambutol. ${ }^{231}$ This study was unblinded and was likely underpowered to detect a difference.

The final RCT involved 119 patients with MAC-pulmonary disease and no previous treatment who were randomised to receive a two-drug (ethambutol and clarithromycin) or threedrug (rifampicin, ethambutol and clarithromycin) regimen. ${ }^{232}$ There was no significant difference in sputum conversion at 1 year between the two groups (33/40 receiving two drugs and 24/32 receiving three drugs converted in the per-protocol arms). However, the study was unblinded and there were a significant number of dropouts in both arms. Additionally the macrolide doses were below standard levels $(200 \mathrm{mg}$ three times a day).

Several non-comparator studies have reported outcome data for a variety of drug regimens used to treat cavitatory and/or nodular bronchiectatic MAC-pulmonary disease. The majority used macrolide-containing regimens and reported culture conversion rates of 13\%-86\%. ${ }^{173}{ }^{189} 233-238$ A large retrospective case series involving 180 patients with nodular bronchiectatic MAC-pulmonary disease reported outcomes using a three-drug predominantly intermittent $(3 \times /$ week) regimen (clarithromycin or azithromycin with rifampicin and ethambutol). ${ }^{237}$ No patient developed macrolide resistance with this regimen and there were no differences in outcome between patients treated with azithromycin versus clarithromycin. While $86 \%$ of patients had culture-converted following 12 months of treatment, 14\% recultured MAC during treatment and 48\% after treatment had ceased. Genotyping subsequently revealed that almost $75 \%$ of isolates were due to reinfection rather than treatment failure. When true relapse occurred it was most commonly in the first 6 months after treatment ceased. A second large retrospective case series that involved 217 patients with nodular bronchiectatic MAC-pulmonary disease, all of whom had not previously received treatment and had clarithromycin-sensitive isolates, reported outcomes using a three-drug regimen prescribed either daily $(n=99)$ or $3 \times /$ week $(n=118) .{ }^{238}$ Modification of the initial regimen was more common in the patients prescribed the daily regimen $(46 \%)$ versus the intermittent regimen $(21 \%)$ $(\mathrm{p}<0.001)$. Treatment was changed most commonly because of visual disturbance on ethambutol and gastrointestinal intolerance on clarithromycin. Rates of culture conversion were $76 \%$ in the daily group versus $67 \%$ in the intermittent group $(\mathrm{p}=0.15)$, and there were no differences in rates of symptomatic or radiographical improvement. Of the 39 (33\%) who failed to convert in the intermittent group, 20 patients were changed to a daily regimen of the same three antibiotics, and of these 6 (30\%) became culture-negative. ${ }^{239}$ While intermittent regimens appear equally effective (accepting that these studies were not RCTs) and better tolerated than daily regimens in patients with nodular bronchiectatic MAC-pulmonary disease, ${ }^{237} 238$ a large multicentre study utilising an intermittent three-drug regimen reported a culture conversion rate of only $4 \%$ after 12 months of treatment in patients with cavitary MAC-pulmonary disease, suggesting that a three-drug regimen should not be used in these patients. $^{189}$

Further observations have been made in the literature based on retrospective, non-comparative data. Sputum conversion may be more successful with higher dose macrolide treatment, ${ }^{240-242}$ but very high doses (clarithromycin $1 \mathrm{~g}$ twice daily and azithromycin $600 \mathrm{mg}$ daily) are poorly tolerated. ${ }^{243} 244$ Rifabutin is less well tolerated than rifampicin, particularly at higher doses. ${ }^{245}$ Development of clarithromycin resistance on treatment is more likely with monotherapy or when given with a quinolone alone. ${ }^{173} 175246$ Rifampicin and rifabutin decrease clarithromycin (but not azithromycin) serum levels by liver enzyme induction, but this has not been shown to affect outcomes and studies have reported similar outcomes with either macrolide. ${ }^{173236237247}$

Failure rates appear higher if patients have had prior treatment ${ }^{248}$ and in those with macrolide resistance. ${ }^{174} 246$ If sputum conversion has not occurred after 6 months of treatment, it is unlikely to occur subsequently. ${ }^{248} 249$ Second-line drugs including those chosen on antibiotic sensitivities may not improve outcome and are often poorly tolerated. ${ }^{250-252}$ One retrospective, uncontrolled study demonstrated 12/41 refractory patients converted their sputum with the addition of moxifloxacin $400 \mathrm{mg}$ once daily. ${ }^{253}$ Other drugs that have been used in a number of studies include clofazimine, linezolid, bedaquiline ${ }^{254-257}$ and nebulised amikacin. The latter has been shown to be beneficial in a proportion of patients with refractory MAC-pulmonary disease in small retrospective case series $^{258} 259$ and more recently in a phase II RCT evaluating a liposomal formulation of amikacin (study published after guideline completion). ${ }^{260} \mathrm{~A}$ phase III RCT evaluating nebulised liposomal amikacin in patients with refractory MAC-pulmonary disease is ongoing. As in vitro data showed synergy between clofazimine and amikacin in 100\% of MAC isolates tested, this treatment combination may be beneficial in patients with refractory MAC-pulmonary disease. ${ }^{261}$ 


\section{Evidence statements}

Treatment with rifampicin, ethambutol and isoniazid reduces the treatment failure/relapse rate compared with rifampicin and ethambutol in individuals with MAC-pulmonary disease. (Evidence level 1-)

Treatment with rifampicin, ethambutol and clarithromycin or azithromycin has demonstrated good outcomes in individuals with MAC-pulmonary disease. (Evidence level 3)

The addition of intramuscular streptomycin to rifampicin, ethambutol and clarithromycin for the first 3 months of treatment for MAC-pulmonary disease improves the rate of culture conversion, but symptom response and radiological outcome are not significantly improved. (Evidence Level 1+)

In nodular bronchiectatic MAC-pulmonary disease, daily and intermittent $(3 \times$ per week) regimens of rifampicin, ethambutol and clarithromycin or azithromycin have equivalent outcomes, but the intermittent ( $3 \times$ per week) regimen is better tolerated. (Evidence level 3)

Intermittent $(3 \times$ per week) antibiotic treatment is associated with poor outcomes in individuals with cavitary MAC-pulmonary disease. (Evidence level 3)

Clarithromycin monotherapy and clarithromycin/quinolonedual therapy regimens for MAC-pulmonary disease are associated with the development of clarithromycin resistance. (Evidence level 3)

\section{Recommendations}

- Clarithromycin-sensitive MAC-pulmonary disease should be treated with rifampicin, ethambutol and clarithromycin or azithromycin using an intermittent $(3 \times$ per week) or daily oral regimen. The choice of regimen should be based on the severity of disease (as defined in table 4) and treatment tolerance. (Grade D)

- An intermittent $(3 \times$ per week $)$ oral antibiotic regimen should not be used in individuals with severe MAC-pulmonary disease (as defined in table 4) or in individuals with a history of treatment failure. (Grade D)

- An injectable aminoglycoside (amikacin or streptomycin) should be considered in individuals with severe MAC-pulmonary disease (as defined in table 4). (Grade D)

- Clarithromycin-resistant MAC-pulmonary disease should be treated with rifampicin, ethambutol and isoniazid or a quinolone, and consider an injectable aminoglycoside (amikacin or streptomycin). (Grade D)

- Nebulised amikacin may be considered in place of an injectable aminoglycoside when intravenous/intramuscular administration is impractical, contraindicated or longer term treatment with an aminoglycoside is required for the treatment of MAC-pulmonary disease. (Grade D)

- Macrolide monotherapy or macrolide/quinolonedual therapy regimens should not be used for the treatment of MAC-pulmonary disease. (Grade D)

- Antibiotic treatment for MAC-pulmonary disease should continue for a minimum of 12 months after culture conversion. (Grade D)

\section{Good practice points}

$\checkmark$ Individuals with clarithromycin-resistant MAC-pulmonary disease should be managed in collaboration with a physician experienced in managing NTM-pulmonary disease.

$\checkmark$ Individuals with a history of treatment intolerance or treatment failure should be managed in collaboration with a physician experienced in managing NTM-pulmonary disease.
Table 3 Suggested antibiotic regimens for adults with M. avium complex-pulmonary disease

M. avium complex-pulmonary

\begin{tabular}{|c|c|}
\hline disease & Antibiotic regimen \\
\hline $\begin{array}{l}\text { Non-severe MAC-pulmonary } \\
\text { disease } \\
\text { (ie, AFB smear-negative } \\
\text { respiratory tract samples, no } \\
\text { radiological evidence of lung } \\
\text { cavitation or severe infection, } \\
\text { mild-moderate symptoms, no } \\
\text { signs of systemic illness) }\end{array}$ & $\begin{array}{l}\text { Rifampicin } 600 \mathrm{mg} 3 \times \text { per week } \\
\text { and } \\
\text { Ethambutol } 25 \mathrm{mg} / \mathrm{kg} 3 \times \text { per week } \\
\text { and } \\
\text { Azithromycin } 500 \mathrm{mg} 3 \times \text { per week or } \\
\text { clarithromycin } 1 \mathrm{~g} \text { in two divided doses } \\
3 \times \text { per week } \\
\text { Antibiotic treatment should continue for a } \\
\text { minimum of } 12 \text { months after culture conversion. }\end{array}$ \\
\hline $\begin{array}{l}\text { Severe MAC-pulmonary } \\
\text { disease } \\
\text { (ie, AFB smear-positive } \\
\text { respiratory tract samples, } \\
\text { radiological evidence of lung } \\
\text { cavitation/severe infection, } \\
\text { or severe symptoms/signs of } \\
\text { systemic illness) }\end{array}$ & $\begin{array}{l}\text { Rifampicin } 600 \mathrm{mg} \text { daily } \\
\text { and } \\
\text { Ethambutol } 15 \mathrm{mg} / \mathrm{kg} \text { daily } \\
\text { and } \\
\text { Azithromycin } 250 \mathrm{mg} \text { daily or clarithromycin } \\
500 \mathrm{mg} \text { twice daily } \\
\text { and consider intravenous amikacin for up to } \\
3 \text { months or nebulised amikacin } \\
\text { Antibiotic treatment should continue for a } \\
\text { minimum of } 12 \text { months after culture conversion. }\end{array}$ \\
\hline $\begin{array}{l}\text { Clarithromycin-resistant } \\
\text { MAC-pulmonary disease }\end{array}$ & $\begin{array}{l}\text { Rifampicin } 600 \mathrm{mg} \text { daily } \\
\text { and } \\
\text { Ethambutol } 15 \mathrm{mg} / \mathrm{kg} \text { daily } \\
\text { and } \\
\text { Isoniazid } 300 \mathrm{mg} \text { (+pyridoxine } 10 \mathrm{mg} \text { ) daily or } \\
\text { moxifloxacin } 400 \mathrm{mg} \text { daily } \\
\text { and consider intravenous amikacin for up to } \\
3 \text { months or nebulised amikacin } \\
\text { Antibiotic treatment should continue for a } \\
\text { minimum of } 12 \text { months after culture conversion. }\end{array}$ \\
\hline
\end{tabular}

\section{SECTION 12B: WHAT ANTIBIOTIC REGIMEN SHOULD BE USED TO TREAT $M$. KANSASII-PULMONARY DISEASE? Evidence summary}

No randomised controlled trials involving individuals with M. kansasii were identified in the literature search. However, four prospective observational studies and several retrospective observational studies reporting detailed antibiotic regimens and associated outcomes were identified.

The four prospective observational studies included 18-173 patients who were treated for 9-18 months with regimens comprising 2-3 drugs. The largest of the prospective studies carried out by the Research Committee of BTS looked at treatment of 173 patients with rifampicin and ethambutol for 9 months of therapy. ${ }^{262}$ There was one treatment failure (positive sputum cultures at 7 and 8 months of treatment) and relapse occurred in $15(9.7 \%)$ patients at a median of 23 months following treatment cessation. Pretreatment bacteriology showed that none of the isolates were resistant to rifampicin or ethambutol, but all were resistant to isoniazid and pyrazinamide. Poor adherence and reinfection were thought to be relevant in some of the cases that relapsed. The next largest prospective study looked at 40 patients treated with isoniazid, rifampicin and ethambutol for 12 months with streptomycin twice weekly for the first 3 months. ${ }^{263}$ There were no treatment failures and relapse occurred in one patient $(2.5 \%) 6$ months postchemotherapy. Mean follow-up in this study was 2.6 years. The third prospective study included 28 patients who were treated with isoniazid, rifampicin and ethambutol for either 12 or 18 months' duration. ${ }^{264}$ There were no treatment failures and one $(3.5 \%)$ relapse in a patient treated for 12 months. Mean follow-up in this study was 1.5 years. The fourth study reviewed the outcomes of 18 patients treated with 
thrice-weekly clarithromycin, rifampicin and ethambutol for 12 months. ${ }^{265}$ There were no treatment failures or relapse following treatment completion. Mean follow-up in the 14 patients that completed treatment was 3.8 years.

The retrospective studies included 35-256 patients who were treated with different multidrug regimens for variable durations of therapy with comparison of treatment failure and relapse rates following treatment. The largest retrospective study involving 256 patients showed higher rates of sputum culture conversion within 4 months of starting treatment and no post-treatment relapses in patients treated with rifampicin-containing regimens compared with those that did not. ${ }^{266} \mathrm{~A}$ similar finding was reported in a study of 162 patients in which there was a more rapid rate of sputum culture conversion in rifampicin-containing regimens. ${ }^{267}$ The smaller retrospective studies reported successful treatment with two and three drug regimens of variable duration with no treatment failure and low rates of relapse. $^{268-272}$

\section{Evidence statement}

Treatment with a rifampicin-based antibiotic regimen is associated with more rapid culture conversion and lower relapse rates in individuals with M. kansasii-pulmonary disease. (Evidence level 3)

Treatment with rifampicin, ethambutol and either isoniazid or a macrolide given for at least 12 months may have lower relapse rates than rifampicin and ethambutol given for 9 months in individuals with $M$. kansasii-pulmonary disease. (Evidence level 3)

\section{Recommendations}

- Rifampicin-sensitive M. kansasii-pulmonary disease should be treated with rifampicin, ethambutol and isoniazid or a macrolide (clarithromycin or azithromycin) using a daily oral regimen. (Grade D)

- Rifampicin-resistant M. kansasii-pulmonary disease should be treated with a three-drug regimen guided, but not dictated by, DST results using a daily oral regimen. (Grade D)

- Antibiotic treatment for M. kansasii-pulmonary disease should continue for a minimum of 12 months after culture conversion. (Grade D)

\section{Good practice points}

$\checkmark \quad$ Individuals with rifampicin-resistant M. kansasii-pulmonary disease should be managed in collaboration with a physician experienced in managing NTM-pulmonary disease.

$\checkmark \quad$ Individuals with a history of treatment intolerance or treatment failure should be managed in collaboration with a physician experienced in managing NTM-pulmonary disease (see table 4).

\begin{tabular}{ll}
\hline $\begin{array}{l}\text { Table } 4 \\
\text { Mycobacterium kansasii-pulmonary disease }\end{array}$ \\
\begin{tabular}{ll} 
M. kansasii-pulmonary disease & Antibiotic regimen \\
\hline Rifampicin-sensitive $M$. kansasii- & Rifampicin $600 \mathrm{mg}$ daily \\
pulmonary disease & and \\
& Ethambutol $15 \mathrm{mg} / \mathrm{kg}$ daily \\
& and \\
& Isoniazid $300 \mathrm{mg}$ (with pyridoxine $10 \mathrm{mg}$ ) daily \\
& or Azithromycin $250 \mathrm{mg}$ daily or Clarithromycin \\
& $500 \mathrm{mg}$ twice daily \\
& Antibiotic treatment should continue for a \\
& minimum of 12 months after culture conversion. \\
\hline
\end{tabular}
\end{tabular}

SECTION 12C: WHAT ANTIBIOTIC REGIMEN SHOULD BE USED TO TREAT M. MALMOENSE-PULMONARY DISEASE? Evidence summary

Two randomised controlled trials and four case series involving individuals with M. malmoense were identified in the literature search.

The first trial involved 106 patients randomised to receive rifampicin, ethambutol and isoniazid or rifampicin and ethambutol for 2 years. ${ }^{213}$ There were no differences in outcome identified between the treatment groups, with the failure of treatment/ relapse rates being low (9\%-12\%). However, the number that completed the allocated treatment and were known to be alive and cured at 5 years was only 38\%-44\%.

The second trial involved 167 patients randomised to receive rifampicin, ethambutol and clarithromycin or rifampicin, ethambutol and ciprofloxacin for 2 years. ${ }^{214}$ In addition, some patients were also randomised to receive $M$. vaccae, but it appeared to have no treatment effect and so the two antibiotic treatment groups were compared irrespective of whether participants received $M$. vaccae immunotherapy. There were no differences in outcome identified between the antibiotic treatment groups, with the failure of treatment/relapse rate being low $(5 \%)$. However, the number that completed the allocated treatment and were known to be alive and cured at 5 years was only $38 \%$ in the clarithromycin group versus $20 \%$ in the ciprofloxacin group.

The non-comparator studies (that involved between 14 and 34 patients) support a treatment effect with three drug regimens (generally, rifampicin and ethambutol in combination with clarithromycin or ciprofloxacin or isoniazid). ${ }^{46273-275}$

\section{Evidence statement}

In individuals with M. malmoense, the most effective and best-tolerated antibiotic regimen appears to be rifampicin, ethambutol and clarithromycin. (Evidence level 1-)

\section{Recommendations}

- M. malmoense-pulmonary disease should be treated with rifampicin, ethambutol and a macrolide (clarithromycin or azithromycin) using a daily oral regimen. (Grade D)

- An injectable aminoglycoside (amikacin or streptomycin) should be considered in individuals with severe M. malmoense-pulmonary disease (ie, AFB smear-positive respiratory tract samples, radiological evidence of lung cavitation/severe infection or severe symptoms/signs of systemic illness). (Grade D)

- Nebulised amikacin may be considered in place of an injectable aminoglycoside when intravenous/intramuscular administration is impractical, contraindicated or longer term treatment with an aminoglycoside is required in the treatment of M. malmoense-pulmonary disease. (Grade D)

- Antibiotic treatment for M. malmoense-pulmonary disease should continue for a minimum of 12 months after culture conversion. (Grade D)

\section{Good practice points}

$\checkmark$ Individuals with a history of treatment intolerance or treatment failure should be managed in collaboration with a physician experienced in managing NTM-pulmonary disease (table 5). 
Table 5 Suggested antibiotic regimens for adults with Mycobacterium malmoense-pulmonary disease

\begin{tabular}{|c|c|}
\hline M. malmoense-pulmonary disease & Antibiotic regimen \\
\hline $\begin{array}{l}\text { Non-severe } \boldsymbol{M} \text {. malmoense- } \\
\text { pulmonary disease } \\
\text { (ie, AFB smear-negative respiratory } \\
\text { tract samples, no radiological evidence } \\
\text { of lung cavitation or severe infection, } \\
\text { mild-moderate symptoms, no signs of } \\
\text { systemic illness) }\end{array}$ & $\begin{array}{l}\text { Rifampicin } 600 \mathrm{mg} \text { daily } \\
\text { and } \\
\text { Ethambutol } 15 \mathrm{mg} / \mathrm{kg} \text { daily } \\
\text { and } \\
\text { Azithromycin } 250 \mathrm{mg} \text { daily or clarithromycin } \\
500 \mathrm{mg} \text { twice daily } \\
\text { Antibiotic treatment should continue for } \\
\text { a minimum of } 12 \text { months after culture } \\
\text { conversion. }\end{array}$ \\
\hline $\begin{array}{l}\text { Severe } M \text {. malmoense-pulmonary } \\
\text { disease } \\
\text { (ie, AFB smear-positive respiratory } \\
\text { tract samples, radiological evidence } \\
\text { of lung cavitation/severe infection or } \\
\text { severe symptoms/signs of systemic } \\
\text { illness) }\end{array}$ & $\begin{array}{l}\text { Rifampicin } 600 \mathrm{mg} \text { daily } \\
\text { and } \\
\text { Ethambutol } 15 \mathrm{mg} / \mathrm{kg} \text { daily } \\
\text { and } \\
\text { Azithromycin } 250 \mathrm{mg} \text { daily or clarithromycin } \\
500 \mathrm{mg} \text { twice daily } \\
\text { and consider intravenous amikacin for up to } \\
3 \text { months or nebulised amikacin } \\
\text { Antibiotic treatment should continue for } \\
\text { a minimum of } 12 \text { months after culture } \\
\text { conversion. }\end{array}$ \\
\hline
\end{tabular}

AFB, acid-fast bacilli.

\section{SECTION 12D: WHAT ANTIBIOTIC REGIMEN SHOULD BE USED TO TREAT M. XENOPI-PULMONARY DISEASE? Evidence summary}

Two randomised controlled trials, one systematic review and eight case series involving individuals with M. xenopi were identified in the literature search.

The first trial involved 42 patients randomised to receive rifampicin, ethambutol and isoniazid or rifampicin and ethambutol for 2 years. ${ }^{213}$ There were no significant differences in outcome identified between the treatment groups. The failure of treatment/relapse rate was $18 \%$ with the two-drug regimen and 5\% with the three-drug regimen; and the number that completed the allocated treatment and were known to be alive and cured at 5 years was $23 \%$ and $10 \%$, respectively. These inconsistent results likely reflect the small number of patients in the study.

The second trial involved 34 patients randomised to rifampicin, ethambutol and clarithromycin or rifampicin, ethambutol and ciprofloxacin for 2 years. ${ }^{214}$ In addition, some patients were also randomised to receive $M$. vaccae, but it appeared to have no treatment effect and so the two antibiotic treatment groups were compared irrespective of whether participants received $M$. vaccae immunotherapy. There were no differences in outcome identified between the treatment groups. The failure of treatment/relapse rate was $24 \%$ with clarithromycin-treated patients and $6 \%$ with ciprofloxacin-treated patients; and the number that completed the allocated treatment and were known to be alive and cured at 5 years was $18 \%$ and $12 \%$, respectively.

The systematic review involved 188 subjects in whom 34 distinct drug regimens were used. ${ }^{276}$ No single drug was found to be superior to another, but quinolone-containing regimens were associated with a greater proportion of relapse-free success.

The non-comparator studies (involving 11-80 patients) included various drug regimens (generally, rifampicin ethambutol and isoniazid; other drugs used were clarithromycin, ciprofloxacin, pyrazinamide, aminoglycosides). ${ }^{277-284}$ Rates of culture conversion and sustained culture negativity were variable, with the largest study reporting that 33/80 (41\%) patients treated with a four-drug regimen remained culture-negative after 3 years of follow-up. ${ }^{284}$

\section{Evidence statement}

In individuals with $M$. xenopi-pulmonary disease, improved outcomes may be achieved using a four-drug antibiotic regimen. (Evidence level 3)

\section{Recommendations}

- M. xenopi-pulmonary disease should be treated with a fourdrug regimen (where tolerated) comprising rifampicin, ethambutol and a macrolide (clarithromycin or azithromycin), with either a quinolone (ciprofloxacin or moxifloxacin) or isoniazid. (Grade D)

- An injectable aminoglycoside (amikacin or streptomycin) should be considered in individuals with severe M. xenopi-pulmonary disease (ie, AFB smear-positive respiratory tract samples, radiological evidence of lung cavitation/ severe infection or severe symptoms/signs of systemic illness). (Grade D)

- Nebulised amikacin may be considered in place of an injectable aminoglycoside when intravenous/intramuscular administration is impractical, contraindicated or longer term treatment with an aminoglycoside is required in the treatment of M. xenopi-pulmonary disease. (Grade D)

- Antibiotic treatment for M. xenopi-pulmonary disease should continue for a minimum of 12 months after culture conversion. (Grade D)

\section{Good practice points}

$\checkmark$ Individuals with a history of treatment intolerance or treatment failure should be managed in collaboration with a physician experienced in managing NTM-pulmonary disease (table 6).

Table 6 Suggested antibiotic regimens for adults with Mycobacterium xenopi-pulmonary disease

\begin{tabular}{|c|c|}
\hline M. xenopi-pulmonary disease & Antibiotic regimen \\
\hline $\begin{array}{l}\text { Non-severe } \boldsymbol{M} \text {. xenopi- } \\
\text { pulmonary disease } \\
\text { (ie, AFB smear-negative respiratory } \\
\text { tract samples, no radiological } \\
\text { evidence of lung cavitation or } \\
\text { severe infection, mild-moderate } \\
\text { symptoms, no signs of systemic } \\
\text { illness) }\end{array}$ & $\begin{array}{l}\text { Rifampicin } 600 \mathrm{mg} \text { daily } \\
\text { and } \\
\text { Ethambutol } 15 \mathrm{mg} / \mathrm{kg} \text { daily } \\
\text { and } \\
\text { Azithromycin } 250 \mathrm{mg} \text { daily or clarithromycin } \\
500 \mathrm{mg} \text { twice daily } \\
\text { and } \\
\text { Moxifloxacin } 400 \mathrm{mg} \text { daily or isoniazid } 300 \mathrm{mg} \\
\text { (+pyridoxine } 10 \mathrm{mg} \text { ) daily } \\
\text { Antibiotic treatment should continue for a } \\
\text { minimum of } 12 \text { months after culture conversion. }\end{array}$ \\
\hline $\begin{array}{l}\text { Severe } \boldsymbol{M} \text {. xenopi-pulmonary } \\
\text { disease } \\
\text { (ie, AFB smear-positive respiratory } \\
\text { tract samples, radiological evidence } \\
\text { of lung cavitation/severe infection, } \\
\text { or severe symptoms/signs of } \\
\text { systemic illness) }\end{array}$ & $\begin{array}{l}\text { Rifampicin } 600 \mathrm{mg} \text { daily } \\
\text { and } \\
\text { Ethambutol } 15 \mathrm{mg} / \mathrm{kg} \text { daily } \\
\text { and } \\
\text { Azithromycin } 250 \mathrm{mg} \text { daily or clarithromycin } \\
500 \mathrm{mg} \text { twice daily } \\
\text { and } \\
\text { Moxifloxacin } 400 \mathrm{mg} \text { daily or isoniazid } 300 \mathrm{mg} \\
\text { (+pyridoxine } 10 \mathrm{mg} \text { ) daily } \\
\text { and consider intravenous amikacin for up to } \\
3 \text { months or nebulised amikacin } \\
\text { Antibiotic treatment should continue for a } \\
\text { minimum of } 12 \text { months after culture conversion. }\end{array}$ \\
\hline
\end{tabular}

AFB, acid-fast bacilli. 


\section{SECTION 12E: WHAT ANTIBIOTIC REGIMEN SHOULD BE USED TO TREAT $M$. ABSCESSUS-PULMONARY DISEASE? Evidence summary}

No randomised controlled trials involving individuals with $M$. abscessus were identified in the literature search. However, five case series reporting detailed antibiotic regimens and associated outcomes were identified.

A study from South Korea evaluated outcomes with a standardised antibiotic regimen in $65 \mathrm{HIV}$-negative patients with ATS defined M. abscessus-PD and evidence of progression during 6-12 months of observation. ${ }^{285}$ Patients received intravenous amikacin and cefoxitin for the first 4 weeks of treatment in combination oral clarithromycin, ciprofloxacin and doxycycline for a median of 12 months. Cefoxitin was poorly tolerated with $39(60 \%)$ having to discontinue it after a median of 22 days due to adverse events including neutropaenia and thrombocytopaenia. Fifty-four (83\%) patients reported an improvement in symptoms and 48 (74\%) showed improved radiological appearances. Thirty-eight $(58 \%)$ culture-converted and remained culture-negative for $>12$ months and 32 (49\%) completed antibiotic therapy and were followed for a median of 12 months without relapse. Rates of long-term culture negativity were significantly lower in patients with clarithromycin-resistant isolates. A subsequent analysis showed that culture conversion and maintenance of negative cultures occurred in $88 \%$ of patients with M. a. massillense compared with only $25 \%$ of patients with $M$. a. abscessus $(\mathrm{p}<0.001) .{ }^{144}$ The difference in outcome is most likely explained by M. a. abscessus isolates having a functional erm(41) gene that confers inducible macrolide resistance. Further data indicated that clarithromycin might be a stronger inducer of erm (41) than azithromycin, ${ }^{145}$ but this has not been a consistent finding. ${ }^{286}$

A study from the USA reported treatment outcomes in 69 patients with ATS-defined M. abscessus-PD that were prescribed ATS/IDSA guidelines-based antibiotic treatment. ${ }^{183}$ Patients were followed for a median of 34 (2-82) months during which $20(29 \%)$ remained culture-positive, 16 (23\%) culture-converted but relapsed, $33(48 \%)$ culture-converted and did not relapse, and $13(19 \%)$ remained culture-negative after cessation of antibiotic treatment for at least 1 year. Patients who underwent surgical resection in addition to medical therapy had higher rates of sustained culture conversion $(57 \%$ vs $28 \%$, $\mathrm{p}=0.022$ ).

A study from South Korea reported outcomes in 41 patients with $M$. abscessus-PD treated with a multidrug regimen containing a macrolide with intravenous amikacin, or a macrolide with intravenous amikacin and cefoxitin or imipenem. ${ }^{217}$ The median duration of treatment with intravenous amikacin was 230 (60-601 days), with cefoxitin or imipenem 83 (25-288) days, and total antibiotic treatment 511 (164-1249) days. All patients were prescribed a macrolide (predominantly clarithromycin). Doxycycline was prescribed to 2 patients, ciprofloxacin to 18 patients and moxifloxacin to 3 patients after finishing parenteral antibiotic therapy. Thirteen (31.7\%) also underwent surgery. Culture conversion was achieved in 33 (81\%) patients at a median of 151 (range 13-860) days. Median follow-up after completion of treatment was 445 (range 0-1443) days during which four patients experienced relapse, with the relapse rate being $3 / 13(23 \%)$ in patients who received one parenteral antibiotic versus $1 / 20(5 \%)$ in those who received two parenteral antibiotics $(p=0.276)$. However, adverse events were more frequent in the two-parenteral than in the one-parenteral antibiotic group $(62.5 \%$ vs $17.6 \%$, $\mathrm{p}<0.05)$. The median duration from the end of treatment to relapse was 523 (range 202-710) days. These encouraging results may reflect that of the M. abscessus isolates tested, almost all were sensitive to the antibiotics prescribed. There was no difference in treatment success or relapse rates between the surgically and non-surgically treated patients. One patient with an isolate resistant to both clarithromycin and amikacin failed to culture-convert. A subsequent study from the same research group using the same treatment approach showed that although the duration of parenteral antibiotic treatment was shorter in patients with $M$. $a$. massiliense than in those with M. a. abscessus (4.7 months vs 7.4 months, $\mathrm{p}=0.006$ ), the treatment success rate was higher in the $M$. a. massiliense group $(95.5 \%$ vs $42.3 \%, \mathrm{p}<0.001) .{ }^{287}$

A case series reported use of intravenous tigecycline as part of a multidrug regimen in patients with $M$. abscessus and $M$. chelonae infections. More than $60 \%$ of patients showed clinical improvement, but treatment was associated with adverse events in more than $90 \%$ of patients, nausea and vomiting being the most common. ${ }^{288}$

Nebulised amikacin was effective in a proportion of patients with refractory M. abscessus-PD in a small retrospective case series, ${ }^{259}$ and data from an RCT evaluating the effects of inhaled liposomal amikacin in this patient group are awaited (study published after guideline completion). ${ }^{260}$

Other antibiotics prescribed for patients with M. abscessus-PD include clofazimine ${ }^{183}$ (noting the synergy between clofazimine and amikacin in vitro), ${ }^{261} 289$ linezolid. ${ }^{183} 255259288$, cotrimoxazole $^{183288}$ and bedaquiline. ${ }^{256}$

M. abscessus produces a broad-spectrum $\beta$-lactamase $\mathrm{Bla}_{\mathrm{Mab}}$, which is the major determinant of $\beta$-lactam resistance. In the zebrafish model of M. abscessus infection, avibactam, a non- $\beta$ lactam $\beta$-lactamase inhibitor, increased survival and decreased abscess formation in zebrafish larvae treated with amoxicillin. ${ }^{290}$ These data suggest that avibactam (which is Food and Drug Administration-approved as a fixed drug combination with ceftazidime for the treatment of adults with complicated intra-abdominal infections) could enhance the effectiveness of $\beta$-lactam treatment in patients with M. abscessus-PD.

In summary, these case series suggest that symptom and radiological improvement tend to occur with intravenous treatment; prolonged intravenous treatment appears to result in better clinical and microbiological outcomes, although toxicity leading to withdrawal of one or more antibiotics is common, and rates of sustained culture conversion appear to be dependent on macrolide susceptibility. It remains unclear whether macrolides should be used to treat isolates demonstrating macrolide resistance, particularly constitutive resistance due to a $23 \mathrm{~S}$ ribosomal RNA point mutation, which confers high-level macrolide resistance, which cannot be overcome in vitro by drug concentrations achievable in man. Furthermore, it is possible that macrolide antibiotics could be deleterious in patients with constitutive macrolide-resistant M. abscessus, as preclinical studies in mice showed that azithromycin treatment may worsen infection, potentially through inhibition of autophagic killing. ${ }^{31}$ Due to the poorer response rates in patients with inducible or constitutive clarithromycin-resistant isolates and the greater efficacy of antibiotics administered through the intravenous route, extending the duration of intravenous antibiotic therapy to 3-6 months in those that can tolerate it may be the most appropriate treatment strategy in this subgroup of patients.

\section{Evidence statements}

Treatment with a multidrug antibiotic regimen including intravenous and oral antibiotics can result in improved symptoms, radiological appearances and microbiological outcomes 
in individuals with M. abscessus-pulmonary disease. (Evidence level 3)

Individuals with macrolide-resistant isolates of M. abscessus may have worse microbiological outcomes than patients with macrolide-sensitive isolates. (Evidence level 3)

\section{Recommendations}

- M. abscessus-pulmonary disease treatment should comprise an initial phase antibiotic regimen (including intravenous and oral antibiotics) followed by a continuation phase antibiotic regimen (including inhaled and/or oral antibiotics). (Grade D)

\section{Initial phase}

- For individuals with M. abscessus isolates that are clarithromycin-sensitive or demonstrate inducible macrolide resistance (see tables 7 and 8), the initial phase antibiotic regimen should include at least a 4-week course of intravenous amikacin, intravenous tigecycline, and (where tolerated) intravenous imipenem, and (where tolerated) oral clarithromycin or oral azithromycin. (Grade D)

- For individuals with M. abscessus complex isolates that demonstrate constitutive macrolide resistance (see tables 7 and 8 ), the initial phase antibiotic regimen should include a minimum 4-week course of intravenous amikacin, intravenous tigecycline and (where tolerated) intravenous imipenem. (Grade D)

- The duration of intravenous treatment should be influenced by the severity of infection, treatment response and tolerance of the regimen. (Grade D)

- To reduce the likelihood of treatment-related nausea and vomiting, antiemetic medication such as ondansetron (note potential for QT interval prolongation) and/oraprepitant should be prescribed to individuals receiving tigecycline and/ or imipenem. (Grade D)

- Nebulised amikacin may be considered in place of intravenous amikacin when intravenous administration is impractical, contraindicated or longer term treatment with an aminoglycoside is required in individuals with M. abscessus-pulmonary disease. (Grade D)

- In the context of amikacin-resistant M. abscessus (ie, MIC $>64 \mathrm{mg} / \mathrm{L}$ or the isolate is known to have a $16 \mathrm{~S}$ rRNA gene mutation conferring constitutive amikacin resistance), intravenous/nebulisedamikacin should be substituted with an alternative intravenous/oral antibiotic. (Grade D)

\section{Continuation phase}

- For individuals with M. abscessus isolates that are clarithromycin-sensitive or demonstrate inducible macrolide resistance (see tables 7 and 8), the continuation phase antibiotic regimen should include nebulised amikacin and a macrolide (oral azithromycin or clarithromycin), in combination with one to three of the following oral antibiotics guided by drug susceptibility and patient tolerance: clofazimine, linezolid, minocycline or doxycycline, moxifloxacin or ciprofloxacin, and co-trimoxazole. (Grade D)

- For individuals with M. abscessus complex isolates that demonstrate constitutive macrolide resistance (see tables 7 and 8), the continuation phase antibiotic regimen should include nebulised amikacin in combination with two to four of the following oral antibiotics guided by drug susceptibility and patient tolerance: clofazimine, linezolid, minocycline or doxycycline, moxifloxacin or ciprofloxacin, and co-trimoxazole. (Grade D)

- In the context of amikacin-resistant M. abscessus (ie, MIC $>64 \mathrm{mg} / \mathrm{L}$ or the isolate is known to have a $16 \mathrm{~S}$ rRNA gene mutation conferring constitutive amikacin resistance), nebulised amikacin should be substituted with an alternative oral antibiotic. (Grade D)

- Antibiotic treatment for M. abscessus-pulmonary disease should continue for a minimum of 12 months after culture conversion. However, individuals who fail to culture-convert may benefit from a long-term suppressive antibiotic regimen. (Grade D)

\section{Good practice point}

$\checkmark \quad$ Individuals with M. abscessus-pulmonary disease should be managed in collaboration with a physician experienced in managing NTM-pulmonary disease.

\section{SECTION 13: IS THERE A ROLE FOR ADJUVANT THERAPIES IN THE MANAGEMENT OF NTM-PULMONARY DISEASE? Evidence summary}

Data relating to three randomised controlled trials of immunotherapy in individuals with NTM-PD were identified.

In the BTS-sponsored randomised controlled trial, patients were randomised to receive $M$. vaccae $(0.1 \mathrm{~mL}$ intradermally 2 monthly for up to 6 months, $n=84)$ or no M. vaccae $(n=86)$, in addition to rifampicin, ethambutol, clarithromycin or ciprofloxacin for 2 years. ${ }^{214}$ No differences in outcome were identified between the immunotherapy and placebo groups.

In a randomised, double-blind, controlled trial performed in the USA, patients with MAC-pulmonary disease were randomised to receive nebulised interferon gamma or placebo, in addition to a standardised antibiotic regimen. ${ }^{189}$ Interim analysis revealed no differences in outcomes between the immunotherapy and placebo groups and the trial was terminated early.

In a randomised controlled trial performed in Cuba, 32 patients with predominantly MAC-pulmonary disease were randomised to receive intramuscular interferon gamma $\left(1 \times 10^{6} \mathrm{IU}\right)$ or placebo daily for 4 weeks, followed by thrice weekly administration for up to 6 months, in addition to a standardised antibiotic

\begin{tabular}{lllll}
\hline \multicolumn{2}{l}{ Table 7} & Interpretation of extended clarithromycin susceptibility results for Mycobacterium abscessus & \\
\hline $\begin{array}{l}\text { Clarithromycin } \\
\text { susceptibility days 3-5 }\end{array}$ & $\begin{array}{l}\text { Clarithromycin } \\
\text { susceptibility day } \mathbf{1 4}\end{array}$ & Genetic implication & M. abscessus subspecies & Macrolide susceptibility phenotype \\
\hline Susceptible & Susceptible & Dysfunctional erm(41) gene & M. a. massiliense & Macrolide susceptible \\
Susceptible & Resistant & Functional erm(41) gene & $\begin{array}{l}\text { M. a. abscessus } \\
\text { M. a. bolletii }\end{array}$ & Inducible macrolide resistance \\
Resistant & Resistant & 235 ribosomal RNA point mutation & Any & $\begin{array}{l}\text { High-level constitutive macrolide } \\
\text { resistance }\end{array}$ \\
\hline
\end{tabular}


Table 8 Suggested antibiotic regimens for adults with Mycobacterium abscessus-pulmonary disease

\begin{tabular}{|c|c|}
\hline M. abscessus & Antibiotic regimen \\
\hline $\begin{array}{l}\text { Clarithromycin } \\
\text { sensitive isolates } \\
\text { or inducible } \\
\text { macrolide-resistant } \\
\text { isolates }\end{array}$ & $\begin{array}{l}\text { Initial phase: } \geq 1 \text { month } † \\
\text { intravenous amikacin } 15 \mathrm{mg} / \mathrm{kg} \text { daily or } 3 \times \text { per week } \neq \\
\text { and } \\
\text { intravenous tigecycline } 50 \mathrm{mg} \text { twice daily } \\
\text { and where tolerated } \\
\text { intravenous imipenem } 1 \mathrm{~g} \text { twice daily } \\
\text { and where tolerated } \\
\text { oral clarithromycin } 500 \mathrm{mg} \text { twice daily or oral azithromycin } \\
250-500 \text { mg daily } \\
\text { Continuation phase: } \\
\text { nebulised amikacin } ¥ \\
\text { and } \\
\text { oral clarithromycin } 500 \mathrm{mg} \text { twice daily or azithromycin } \\
250-500 \text { mg daily } \\
\text { and } 1-3 \text { of the following antibiotics guided by drug } \\
\text { susceptibility results and patient tolerance: } \\
\text { oral clofazimine } 50-100 \mathrm{mg} \text { daily§ } \\
\text { oral linezolid } 600 \mathrm{mg} \text { daily or twice daily } \\
\text { oral minocycline } 100 \mathrm{mg} \text { twice daily } \\
\text { oral moxifloxacin } 400 \mathrm{mg} \text { daily } \\
\text { oral co-trimoxazole } 960 \mathrm{mg} \text { twice daily }\end{array}$ \\
\hline $\begin{array}{l}\text { Constitutive } \\
\text { macrolide-resistant } \\
\text { isolates }\end{array}$ & $\begin{array}{l}\text { Initial phase: } \geq 1 \text { month } t \\
\text { intravenous amikacin } 15 \mathrm{mg} / \mathrm{kg} \text { daily or } 3 \times \text { per week } \neq \\
\text { and } \\
\text { intravenous tigecycline } 50 \mathrm{mg} \text { twice daily } \\
\text { and where tolerated } \\
\text { intravenous imipenem } 1 \mathrm{~g} \text { twice daily } \\
\text { Continuation phase: } \\
\text { nebulised amikacin } ¥ \\
\text { and } \\
2-4 \text { of the following antibiotics guided by drug susceptibility } \\
\text { results and patient tolerance: } \\
\text { oral clofazimine } 50-100 \text { mg daily§ } \\
\text { oral linezolid } 600 \mathrm{mg} \text { daily or twice daily } \\
\text { oral minocycline } 100 \mathrm{mg} \text { twice daily } \\
\text { oral moxifloxacin } 400 \mathrm{mg} \text { daily } \\
\text { oral co-trimoxazole } 960 \mathrm{mg} \text { twice daily }\end{array}$ \\
\hline
\end{tabular}

tDue to the poorer response rates in patients with inducible or constitutive macrolide-resistant isolates and the greater efficacy of antibiotics administered through the intravenous route, extending the duration of intravenous antibiotic therapy to 3-6 months in those that can tolerate it may be the most appropriate treatment strategy in this subgroup of patients.

$¥$ Substitute intravenous/nebulised amikacin with an alternative antibiotic if the $M$. abscessus is resistant to amikacin (ie, minimum inhibitory concentration $>64 \mathrm{mg} / \mathrm{L}$ or known to have a $16 \mathrm{~S}$ rRNA gene mutation conferring constitutive amikacin resistance).

$\S$ Start clofazimine during the initial phase of treatment if tolerated as steady-state serum concentrations may not be reached until $\geq 30$ days of treatment.

\section{Evidence statement}

Intramuscular interferon gamma resulted in a significant improvement in a novel composite clinical/bacteriological/ radiological endpoint in individuals with predominantly MAC-pulmonary disease receiving a standardised antibiotic regimen of daily oral azithromycin, rifampicin, ethambutol and ciprofloxacin. (Evidence level 1-)

Nebulised interferon gamma and subcutaneous M. vaccae do not improve outcomes in individuals with NTM-pulmonary disease. (Evidence level 1-)

\section{Recommendations}

- Interferon gamma is not recommended as adjuvant therapy in individuals with NTM-pulmonary disease without a defined immunodeficiency affecting intrinsic interferon gamma signalling. (Grade D)

- M. vaccae is not recommended as adjuvant therapy in individuals with NTM-pulmonary disease. (Grade D)

\section{SECTION 14: WHAT INVESTIGATIONS SHOULD BE PERFORMED DURING TREATMENT OR FOLLOWING TREATMENT FOR NTM-PULMONARY DISEASE? \\ Evidence summary}

There are no prospective controlled trials evaluating which investigations should be performed during treatment or following treatment for NTM-PD. However, a variety of outcome measures have been used to assess the efficacy, safety and tolerability of treatment within the randomised controlled trials and non-comparator studies involving patients with NTM-PD.

\section{Microbiological outcomes}

The conventional microbiological outcomes are smear status, culture conversion and relapse (box 2). In a recent retrospective study of 180 patients receiving standard therapy for nodular bronchiectatic MAC lung disease, a reduction in a semiquantitative sputum culture score from baseline to month 2 of treatment was highly predictive of subsequent sputum culture conversion and significantly correlated with symptom response (cough, fatigue, fever, haemoptysis) and radiographical improvement. ${ }^{185}$

In a prospective randomised controlled treatment trial, deaths attributable to the NTM infection were more frequent in those who remained culture-positive after 12 months' treatment. ${ }^{214}$

\section{Box 2 Definitions for microbiological outcomes}

an improvement in symptoms, radiology and microbiology) was achieved by $13 / 18$ (72\%) participants receiving Interferon gamma versus $5 / 14(36 \%)$ receiving placebo $(\mathrm{p}=0.037)$ after 6 months of treatment and the improvement was maintained for 12 months following treatment cessation. While these results are encouraging, the use of a novel composite endpoint suggests that further supporting data are required before this treatment approach can be recommended.

No studies were identified that evaluate whether vitamin D supplementation enhances host immunity and improves outcomes in patients with NTM-PD.

No studies were identified that evaluate whether nutritional supplementation improves outcomes in nutritionally depleted patients with NTM-PD. 


\section{Evidence statement}

Deaths attributable to NTM infection are more frequent in patients who remain culture-positive despite 12 months of NTM treatment. (Evidence level 3)

A reduction in a semiquantitative sputum culture score from baseline to month 2 of treatment is predictive of subsequent treatment success in patients receiving standard therapy for nodular bronchiectatic MAC-pulmonary disease. (Evidence level 3)

\section{Recommendations}

- Sputum samples should be sent for mycobacterial culture every 4-12 weeks during treatment and for 12 months after completing treatment to assess the microbiological response. (Grade D)

- If there is doubt about persisting NTM infection despite negative sputum cultures, a CT-directed bronchial wash should be performed to assess the microbiological response to treatment. (Grade D)

- In individuals who are unable to expectorate sputum, a CT scan followed by a CT-directed bronchial wash after 6 and 12 months of treatment can be used to assess the microbiological response to treatment. (Grade D)

\section{Good practice point}

$\checkmark$ In individuals who are unable to spontaneously expectorate sputum and in whom CT-directed bronchial washings are not feasible, induced sputum samples should be sent for mycobacterial culture every 4-12 weeks during treatment and for 12 months after completing treatment to assess the microbiological response.

\section{Radiological outcomes}

Chest X-rays and CT images can be used to evaluate change in bronchiectasis (severity and distribution), bronchial wall thickening, mucus plugging, tree-in-bud opacity, consolidation, nodular change and cavitation (number, size, wall thickness, intracavity debris). While there are no prospective studies indicating which radiological features are most informative during and following treatment for NTM-pulmonary disease, a large retrospective case series involving patients on treatment for MAC-pulmonary disease showed that patients that culture-converted demonstrated significantly greater improvements in chest $\mathrm{X}$-ray and CT scores than non-converters, and change in semiquantitative culture scores correlated with improvement in chest X-ray and CT scores during the early phase of therapy. ${ }^{185}$

Abnormalities on plain chest X-ray, including the persistence of cavities, have been reported in up to $75 \%$ of patients at the end of treatment, even among those who had shown a satisfactory clinical response and culture-converted. ${ }^{214} 274$

\section{Evidence statement}

In individuals receiving treatment for MAC-pulmonary disease, patients who culture-converted demonstrated significantly greater improvements in chest X-ray and CT scores than non-converters. (Evidence level 3)

Individuals in whom satisfactory clinical progress and sputum culture conversion have been demonstrated may still have radiological evidence of lung cavitation after completing treatment. (Evidence level 3)

\section{Recommendation}

- A CT scan should be performed shortly before starting NTM treatment and at the end of NTM treatment to document the radiological response to treatment. (Grade D)

\section{Good practice point}

$\checkmark$ During the course of treatment for NTM-pulmonary disease, more frequent radiological monitoring may be indicated in selected individuals.

\section{CLINICAL OUTCOMES}

While there are no prospective studies indicating which clinical endpoints are most informative during and following treatment for NTM-pulmonary disease, a large retrospective case series involving patients on treatment for MAC-pulmonary disease provides some insights. ${ }^{185}$ Almost all patients presented with cough, sputum production and fatigue, and patients whose cough improved while prescribed NTM treatment were more likely to culture-convert $(\mathrm{p}<0.001)$. Furthermore, the presence of fatigue, haemoptysis and fever was significantly correlated with semiquantitative culture results when evaluated monthly throughout the follow-up period $(\mathrm{p}<0.003$, correlation coefficient range $0.1-0.2$ ).

There are no specific quality of life tools that have been developed specifically for patients with NTM-PD. However, in a study conducted in Japan in patients with MAC-pulmonary disease, stepwise multiple regression analysis showed that CT scan findings (consolidation, cavitation and lobar volume decrease) correlated with the St George's Respiratory Questionnaire (SGRQ) total score, ${ }^{292}$ suggesting that the SGRQ may be appropriate for use in patients with NTM-PD associated with COPD or bronchiectasis.

Deaths due to mycobacterial disease and a number of composite clinical outcomes were used in the BTS-sponsored randomised controlled trials. ${ }^{213} 214$ In practice these endpoints are challenging due to the difficulty of confidently ascribing causation in the context of frequent comorbidity.

\section{Evidence statement}

Changes in semiquantitative culture score and symptom response (particularly cough) are significantly correlated in individuals receiving antibiotic treatment for nodular bronchiectatic MAC-pulmonary disease. (Evidence level 3)

\section{Recommendation}

- A detailed assessment of pulmonary and systemic symptoms should be recorded at each clinical review. (Grade D)

\section{Good practice point}

$\checkmark$ A more detailed clinical assessment may include measurements of body weight, spirometry and systemic inflammatory markers (ESR and CRP).

\section{THERAPEUTIC DRUG MONITORING}

In a retrospective single-centre study of patients receiving a standard three-drug regimen for MAC-pulmonary disease, there was no association between low plasma clarithromycin concentrations and treatment outcomes. ${ }^{293}$ Although clarithromycin levels in plasma were below the target of $2 \mu \mathrm{g} / \mathrm{mL}$ in $97 \%-100 \%$ of cases depending on the frequency of drug administration, $74 \%$ of patients who completed at least 12 months of treatment culture-converted, 79\% showed symptomatic improvement and appearances on high-resolution CT improved in 66\%. The apparent discrepancy in these results may reflect that drug concentrations achieved within tissues and alveolar macrophages are much higher than those achieved in plasma.

In a retrospective single-centre study of patients undergoing treatment for MAC-pulmonary disease, peak serum 
concentrations below the target range were frequent for ethambutol (48\%), clarithromycin (56\%) and azithromycin (35\%). ${ }^{170}$ Concurrent administration of rifampicin led to $68 \%, 23 \%$ and $10 \%$ decreases in peak serum concentrations of clarithromycin, azithromycin and moxifloxacin, respectively, whereas rifabutin increased azithromycin levels. Similar effects of rifampicin on clarithromycin concentrations were reported in a small prospective study examining the pharmacokinetic interactions of drugs commonly prescribed to treat MAC-pulmonary disease. ${ }^{294}$

\section{Evidence statement}

Drug-drug interactions frequently occur between drugs prescribed to treat NTM-pulmonary disease. (Evidence level 3)

Although rifampicin reduces the peak serum concentration of clarithromycin, this effect has not been shown to influence treatment outcome. (Evidence level 3)

\section{Recommendations}

- Therapeutic drug monitoring (other than for aminoglycosides) should not be performed routinely in individuals' prescribed antibiotic therapy for NTM-pulmonary disease. (Grade D)

- When aminoglycosides are administered, serum levels and the serum creatinine must be monitored and aminoglycoside dosing adjusted according to local policies. (Grade D)

\section{Good practice point}

$\checkmark \quad$ Therapeutic drug monitoring can be considered in individuals in whom gastrointestinal malabsorption, drug-drug interactions or suboptimal adherence may be adversely affecting treatment response.

\section{MONITORING FOR DRUG TOXICITY}

Aminoglycosides can result in permanent auditory, vestibular and renal toxicity. While the risk of adverse events is greatest when aminoglycoside levels exceed recommended levels, the risk of toxicity is also likely to vary according to the aminoglycoside prescribed, duration of exposure, route of administration and host susceptibility. In a prospective randomised study of two aminoglycoside dosing regimens $(15 \mathrm{mg} / \mathrm{kg}$ per day or $25 \mathrm{mg} / \mathrm{kg}$ three times per week with doses adjusted to target drug concentrations), the size of dose or frequency of administration was not associated with adverse events. ${ }^{295}$ However, the risk of ototoxicity (found in 32/87 participants) was associated with older age, duration of treatment and cumulative dose. Vestibular toxicity (found in $8 / 87$ participants) usually resolved and renal toxicity (found in 13/87 participants) was mild and reversible in all cases. Of amikacin, kanamycin and streptomycin, the later was least associated with hearing loss. Mutations in mitochondrial DNA may confer greater risk of aminoglycoside ototoxicity, ${ }^{296}$ although there is currently no evidence that prescribing practice based on the presence/absence of mitochondrial DNA mutations reduces the risk of aminoglycoside-related adverse events in patients with NTM-PD.

Ethambutol can cause optic neuritis and retrobulbar neuritis that present with blurred vision, decreased acuity, central scotomas, impaired red-green colour discrimination and peripheral visual field defects. ${ }^{297}$ The risk of developing ethambutol-related ocular toxicity is greater with NTM than TB treatment as the duration of treatment is longer. In a retrospective analysis, ethambutol-related ocular toxicity was diagnosed in $8 / 139(6 \%)$ patients receiving daily therapy $(25 \mathrm{mg} / \mathrm{kg}$ for the first 2 months followed by $15 \mathrm{mg} / \mathrm{kg}$ for the remainder of therapy) compared with $0 / 90$ cases in patients prescribed intermittent therapy (ethambutol $25 \mathrm{mg} / \mathrm{kg} \mathrm{3 \times}$ per week). ${ }^{297}$ As ethambutol is largely excreted unchanged by the kidneys, clearance is reduced and the risk of toxicity is increased in patients with renal impairment, ${ }^{298}$ thus dosing needs to be adjusted in these circumstances. ${ }^{299}$

Adverse effects occur commonly in patients treated with macrolide antibiotics. In a non-randomised prospective study of predominantly elderly patients prescribed azithromycin $600 \mathrm{mg}$ per day for 4-6 months to treat MAC-pulmonary disease or $M$. abscessus complex-PD, 82\% experienced gastrointestinal side effects and 26\% developed hearing impairment. ${ }^{247}$ Adverse effects were associated with higher drug serum levels and dose reduction to $300 \mathrm{mg}$ daily resulted in resolution of most adverse effects. More recently, concerns have been raised about cardiac side effects in patients treated with azithromycin that relate to potential prolongation of the QT interval, ${ }^{300}$ although this specific adverse event has not been reported in NTM treatment studies to date.

A comprehensive list of NTM treatment related adverse events is found within the NTM drug monograph (section 18).

\section{Evidence statements}

Aminoglycosides used to treat NTM-pulmonary disease can result in auditory, vestibular and renal side effects. (Evidence level 3)

Ethambutol used to treat NTM-pulmonary disease can result in ocular toxicity, particularly with daily dosing. (Evidence level 3)

The excretion of ethambutol is reduced in patients with renal dysfunction. (Evidence level 3)

Azithromycin used to treat NTM-pulmonary disease can cause gastrointestinal side effects, tinnitus and hearing loss (particularly with higher doses in elderly patients). (Evidence level 3)

\section{Recommendations}

- When aminoglycosides are administered serum levels and the serum creatinine must be monitored and aminoglycoside dosing adjusted according to local policies. (Grade D)

- Audiometry should be considered before starting aminoglycosides and intermittently during treatment (frequency according to perceived risk and symptoms). Patients should be informed to stop aminoglycoside treatment immediately and to inform the prescriber if they develop tinnitus, vestibular disturbance or hearing loss. (Grade D)

- Assess visual acuity and colour vision before starting ethambutol and advise patients to stop treatment immediately and inform the prescriber if changes in visual acuity or colour vision occur. (Grade D)

- Serum ethambutol levels should be measured in patients with renal dysfunction. (Grade D)

\section{Good practice points}

$\checkmark \quad$ The frequency/type of toxicity monitoring required during NTM treatment is dependent on the drug regimen. Treatment-related adverse events and suggested toxicity monitoring protocols are outlined in the NTM antibiotic treatment monograph (section 18).

$\checkmark$ Audiometry should be considered before starting azithromycin or clarithromycin and intermittently during treatment (frequency according to perceived risk and symptoms) and advise individuals to stop treatment immediately and inform the prescriber if they develop tinnitus, vestibular disturbance or hearing loss.

$\checkmark$ Perform an ECG before, and 2 weeks after, starting drugs (such as azithromycin or clarithromycin) that are known to prolong the QT interval. 


\section{SECTION 15: ARE THERE DIFFERENCES IN OUTCOME BETWEEN INDIVIDUALS WITH NTM-PULMONARY DISEASE TREATED IN SPECIALIST VERSUS NON-SPECIALIST CARE SETTINGS? \\ Evidence summary}

There are no randomised controlled trials that address whether there is a difference in outcome between individuals with NTM-PD treated in specialist versus non-specialist care settings. However one study has evaluated treatment approaches between specialists and non-specialists and another study evaluated whether there were differences in guideline-compliant antibiotic prescribing between physician specialties.

In a study performed in Canada and the USA, 46 Canadian respiratory physicians and 19 experts in NTM management were surveyed. ${ }^{301}$ Experts and non-experts agreed in many areas. However, non-experts estimated fewer patients with positive cultures had disease ( $30 \%$ vs $50 \%, \mathrm{p}=0.02)$, used intensive-guideline therapy less often in new cases $(50 \%$ vs $79 \%, \mathrm{p}=0.02)$ and perceived a lower success rate with guidelines therapy $(65 \%$ vs $75 \%, \mathrm{p}=0.047)$. Response ranges were also wider for non-experts, significantly so for selection of intensive-guidelines therapy in new $(p=0.01)$ and recurrent $(p=0.04)$ cases.

In a study performed in the USA, physicians involved in treating patients with NTM-PD were asked to extract medical record data on the last four patients they had treated with MAC or M. abscessus-PD. ${ }^{302}$ Three hundred forty-nine physicians provided data on 915 patients, including 744 (81\%) with MAC, 174 (19\%) with M. abscessus and 3 with both. Physicians treated 411 (55\%) patients with MAC and 76 (44\%) patients with $M$. abscessus. Only $13 \%$ of antibiotic regimens prescribed to patients with MAC were ATS/IDSA guideline-compliant, with $56 \%$ of regimens not including a macrolide and $30 \%$ associated with an increased risk of developing macrolide resistance. While more regimens prescribed to patients with MAC by respiratory specialists were more likely to be ATS/IDSA guideline-compliant (18\%) compared with those prescribed by infectious diseases physicians $(10 \%)$ or general practice/internalmedicine physicians (9\%), respiratory specialists also more frequently prescribed regimens associated with an increased risk of macrolide resistance (40\%), versus infectious disease $23 \%$ or general practice/internal medicine physicians (23\%). Among patients with M. abscessus, 64\% of regimens did not include a macrolide and $18 \%$ associated with an increased risk of macrolide resistance.

\section{Evidence statements}

Experts are more likely than non-experts to make a diagnosis of NTM-pulmonary disease in patients with MAC-positive cultures. (Evidence level 3)

Experts are more likely than non-experts to use intensive guideline-based antibiotic regimens in new cases of NTM-pulmonary disease. (Evidence level 3)

\section{Recommendation}

- Individuals with NTM-pulmonary disease should be managed in collaboration with a physician experienced in managing NTM-pulmonary disease. (Grade D)

\section{SECTION 16: WHAT IS THE ROLE OF SURGERY IN THE TREATMENT OF NTM-PULMONARY DISEASE? Evidence summary}

Antimicrobial chemotherapy is the mainstay of treatment for NTM-PD, but even in expert centres and in the setting of controlled trials a significant proportion of patients will not achieve cure. Surgery for NTM-PD has been described by a number of centres internationally. Several retrospective case series have been published reporting surgical outcomes in patients with NTM-PD, ${ }^{303-322}$ the largest involving 236 patients. ${ }^{307}$ Surgical experience is greatest with MAC-pulmonary disease, but M. abscessus complex, ${ }^{307}$ M. kansasii ${ }^{319}$ and M. xenopi$\mathrm{PD}^{312}$ have also been the subject of moderate-size studies (32, 35 and 57 patients, respectively). The available data suggest that surgery can be an important adjunct to antimicrobial treatment in carefully selected cases. The sputum culture conversion rate following surgery is in the range of $85 \%-100 \%$ and the longterm relapse rate is probably under $10 \% .^{304-322}$ However, the data also show that even in expert centres surgery can be associated with significant morbidity (including bronchopleural fistula, respiratory failure, sepsis, bleeding, reoperation, prolonged air leak and arrhythmia), indicating that the decision to proceed with surgery must be subject to a rigorous risk-benefit analysis within a multidisciplinary setting, preferably in an expert centre.

While there is no strong evidence base to guide patient selection, several factors have emerged from the literature that ought to be considered.

\section{Microbiological}

The main indications for surgery are culture positivity despite 6-12 months of treatment with an appropriate NTM antibiotic regimen or relapse after completing treatment. The potential impact of drug resistance and/ordrug intolerance must be considered because of the requirement for ongoing antibiotic treatment following surgery.

\section{Disease status}

Surgery should be considered where there is cavitary disease that is limited in site and extent. Lobectomy and bilobectomy are the procedures of choice, although pneumonectomy is appropriate where there is unilateral destroyed lung and is described with varying success rates from different centres. ${ }^{304} 307315$ Disease-related local complications such as significant haemoptysis or aspergilloma may also favour surgical intervention.

\section{Cardiopulmonary fitness}

The patient must have sufficient physiological reserve to tolerate lung resection. This is an important consideration given the likelihood of underlying chronic lung disease in this patient population. The assessment of patients for cardiothoracic surgery will likely vary according to local guidelines, but it has previously been suggested that the BTS/ACCP guidelines for consideration of lung cancer resection be followed in this setting. ${ }^{32} 324$ Optimal assessment will likely include exclusion of significant cardiac disease, spirometry including lung volumes and gas transfer, cardiopulmonary exercise testing and ventilation perfusion scanning. Smoking cessation should be recommended and supported.

\section{Nutrition}

The authors of surgical case series frequently emphasise the importance of nutritional status to good surgical outcome. ${ }^{306307312}$ It is appropriate to measure and monitor patients' BMI, to perform a thorough nutritional assessment (preferably by a trained dietitian) and to optimise nutritional status with dietary supplementation. Factors that may influence nutritional status such as tolerance of chemotherapy, gastro-oesophageal reflux disease, alcohol intake, smoking and recreational drug abuse should also be addressed. 


\section{Surgery and antimycobacterial antibiotic treatment}

Multidrug antimycobacterial antibiotic treatment should be given prior to surgical intervention, but there is no evidence to guide optimal duration. In practice, antimycobacterial antibiotic treatment is usually prescribed for 1 year following surgery, if it is assumed that culture conversion occurred at the time of surgery.

In the context of a solitary MAC nodule being removed, often following lung resection for possible neoplasia or during lung volume reduction surgery, experience suggests that the procedure may be curative and NTM antibiotic treatment is not necessary. ${ }^{325}$

\section{Surgical experience}

Increasing surgical experience reduces operative mortality in patients with NTM-PD, with operative mortality reducing from $7.1 \%$ to $0.6 \%$ between 1983 and 2006 in the largest case series to date. ${ }^{307} \mathrm{~A}$ detailed analysis of surgical approach and technique is outside the scope of this guideline. However, it is hoped that with the advent of minimally invasive procedures the option of lung resection surgery will become increasingly feasible for patients with NTM-PD. ${ }^{303}$ Given the risk of significant morbidity $(15 \%-50 \%)$ associated with surgery ${ }^{304-322}$ and the relatively low case volume in the UK, patients should be referred to experienced regional or national centres. Given the requirement for ongoing medical input for these patients both on an inpatient and long-term outpatient basis, surgery should ideally be planned in an expert multidisciplinary environment and carried out in a centre with both medical and surgical expertise in the management of NTM-PD.

\section{Evidence statements}

Lung resection surgery in selected individuals with NTM-pulmonary disease can result in a high sputum conversion rate and a low relapse rate. (Evidence Level 3)

Lung resection surgery for NTM-pulmonary disease can be associated with significant morbidity and the complication rate is higher with more extensive resection/pneumonectomy. (Evidence level 3)

Increased operator/centre expertise in performing lung resection surgery for NTM-pulmonary disease is associated with reduced postoperative morbidity and mortality. (Evidence Level 3)

\section{Recommendations}

- The role of lung resection surgery in the management of NTM-pulmonary disease should be considered at the time of diagnosis and revisited in individuals who develop refractory disease. (Grade D)

- Lung resection surgery for NTM-pulmonary disease may be indicated in individuals with localised areas of severe disease. (Grade D)

- Lung resection surgery for NTM-pulmonary disease should only be performed following expert multidisciplinary assessment in a centre experienced in managing individuals with NTM-pulmonary disease. (Grade D)

- Individuals with NTM-pulmonary disease should be established on antibiotic treatment prior to lung resection surgery and should continue treatment for 12 months after culture conversion. (Grade D).

- Following resection of a solitary NTM nodule in an individual with no other features of NTM-pulmonary disease, antibiotic treatment is not usually required. (Grade D)

\section{Good practice points}

$\checkmark$ Individuals with NTM-pulmonary disease in whom lung resection surgery is being considered should have a comprehensive assessment of cardiopulmonary status in line with current guidance for lung cancer resection.

$\checkmark \quad$ Nutritional status should be optimised prior to lung resection surgery.

\section{SECTION 17: DOES NTM INFECTION AFFECT AN INDIVIDUAL'S SUITABILITY FOR LUNG TRANSPLANTATION? Evidence summary}

The risk of developing NTM infection is likely to be increased in lung transplant recipients due to the effects of powerful immunosuppression. NTM-PD or soft tissue/disseminated infection can arise either de novo or by resurgence of an organism that was present prior to surgery. Treatment of NTM-PD or soft tissue/disseminated infection after lung transplantation requires a complex regimen of intravenous and oral antibiotics, usually for a prolonged period of time, and requires careful monitoring due to the risk of drug-drug interactions. ${ }^{326}$ The current guidelines on selection of lung transplant candidates from the International Society of Heart and Lung Transplantation list 'colonisation' of a potential candidate with highly resistant mycobacteria as a relative contraindication to lung transplantation. ${ }^{327}$ However, the evidence base at the time of publication was very limited and more case series and cohort studies have since been published to inform decision-making.

In a single-centre cohort study of 237 lung transplant recipients, $53(22 \%)$ isolated NTM. ${ }^{328}$ The the most common organism was MAC (70\%), followed by M. abscessus $(9 \%)$ and M. gordonae (8\%). Overall mortality was no different in the NTM group compared with the non-NTM group and only 6/53 who isolated NTM required treatment. Of these, four developed persistent disease within the wound (three due to $M$. abscessus, one of whom died of disseminated infection), one did not isolate NTM following antibiotic treatment and one developed persistent infection but had no features of disease. Only five patients isolated NTM pretransplantation (four with MAC and one with M. scrofulaceum), three of whom did not grow NTM post-transplant.

In a study of 201 lung transplant recipients, 36 (18\%) isolated NTM following the procedure, ${ }^{329}$ with $75 \%$ of isolations deemed to be infection only and 25\% disease by ATS/IDSA criteria. NTM infection was associated with an increased risk of death (HR 2.61) in multivariate models, but the cause of death in this series was due to infections other than NTM. De novo NTM acquisition occurred in 14\% of patients. Six of the 165 recipients in the non-NTM group had cultured NTM pretransplant, none of whom isolated NTM post-transplant.

In a large single-centre experience over a 13 -year period, $20 \%$ of 146 patients with CF referred for lung transplantation had isolated NTM pretransplant. ${ }^{330}$ This group had a five times increased risk (OR 5.03) of culturing NTM after transplantation and a six times increased risk of developing invasive disease compared with recipients who had not isolated NTM before transplant. MAC and M. abscessus were the the most common organisms accounting for $45 \%$ and $41 \%$ of isolates, respectively. Twenty-five per cent of patients developed invasive disease, predominantly those with $M$. abscessus. Overall survival in recipients with NTM was similar to the non-NTM group and none of the patients who developed de novo NTM infection post-transplant developed invasive disease. Of the seven patients who isolated M. abscessus pre-treatment, five cultured 
the organism post-transplant, of whom two developed surgical wound infections that were treated successfully with prolonged courses of antibiotics.

In a survey of 31 lung transplant programmes with a collective experience of 5200 lung transplants, 17 patients with post-transplant M. abscessus infection were identified, ${ }^{331}$ two of whom had cultured the organism before transplant and the remainder isolated it de novo following lung transplant. The two reinfected patients developed extensive soft tissue infection and died 2 and 14 months post-transplant, respectively, but not from disseminated M. abscessus. Of the patients with post-transplant de novo infections, 66\% eradicated M. abscessus following antibiotic therapy.

In a case series of five paediatric patients infected with $M$. abscessus prior to lung transplantation, three patients were infected with M. a. abscessus, one with M. massiliense and one with $M$. bolletii. ${ }^{332}$ The patients with $M$. massiliense and $M$. bolletii survived long term after transplant. However, all three patients with $M$. a. abscessus encountered complications with wound infections that required prolonged antibiotic treatment. One patient died 21 days following surgery and the remaining two lived to 30 months and more than 4 years, respectively. The authors suggested that postoperative risk could be stratified through subspeciating the $M$. abscessus complex and that the NTM load before transplant should be lowered with 6 months of antibiotic treatment prior to listing.

In a case series of four patients infected with M. abscessus prior to lung transplantation, three developed extensive local soft tissue and disseminated disease following transplantation and required ${ }^{333}$ prolonged treatment including intravenous antibiotics and immunoglobulin. When the series was reported these patients were alive at 3, 5 and 7 years post-transplant, showing that long-term survival with $M$. abscessus disease post-transplant can be achieved. The fourth patient did not grow M. abscessus again after transplant.

In a report from the Danish CF centre involving five smear-positive patients with $M$. abscessus disease prior to lung transplantation, ${ }^{334}$ two died early in the postoperative course due to non-NTM-related causes, two developed deep surgical wound infections due to M. abscessus and one remained culture-negative for a year following surgery and then reisolated M. abscessus but remained well.

An updated report from the Chapel Hill group described their cumulative experience with 13 patients with CF infected with M. abscessus prior to lung transplantation. ${ }^{335}$ Only three patients developed postoperative complications due to M. abscessus and all responded to prolonged antibiotic therapy. The overall survival in the whole cohort was $50 \%$ at 5 years and was not statistically different from a cohort of contemporaneous lung transplant recipients without $M$. abscessus infection.

\section{Evidence statements}

NTM are isolated from respiratory tract cultures in approximately $20 \%$ of individuals following lung transplantation. However, the majority of individuals do not develop NTM-pulmonary disease. (Evidence level 3)

Pretransplant isolation of $M$. abscessus is associated with an increased risk of post-transplant soft tissue infection and pulmonary infection/disease. (Evidence level 3)

The isolation of M. abscessus post-transplantation is associated with significant morbidity, but NTM-related mortality does not appear to be increased. (Evidence level 3)

\section{Recommendations}

- Individuals being considered for lung transplantation referral should be assessed for evidence of NTM-pulmonary disease. (Grade D)

- Isolation of NTM organisms including M. abscessus in potential lung transplant candidates should not preclude referral and assessment for lung transplantation. (Grade D)

- Potential lung transplant candidates with evidence of NTM-pulmonary disease should be treated whenever possible prior to listing to either eradicate the organism or lower bacterial load. (Grade D)

- Individuals with previous or current M. abscessus infection or disease who are listed for lung transplantation should be counselled about the high postoperative risk of developing invasive and disseminated NTM disease, which causes significant morbidity and necessitates prolonged treatment with a multidrug antibiotic regimen. (Grade D)

\section{Good practice points}

$\checkmark$ Individuals with NTM-pulmonary disease should demonstrate an ability to tolerate optimal antibiotic therapy before listing for lung transplantation.

$\checkmark$ Progressive NTM-pulmonary disease despite optimal antibiotic therapy is likely to be a contraindication to listing for lung transplantation. 


\section{SECTION 18: NTM DRUG MONOGRAPH}

The treatment of NTM-PD involves complex drug regimens that are commonly associated with intolerance and toxicity. The purpose of this monograph is to facilitate antibiotic prescribing in people with NTM-PD, but it should only be used in conjunction with, and not as a substitute for, local/national prescribing formularies. The monograph also provides guidance on the type and frequency of monitoring for drug toxicity, which must, however, be determined on a case-by-case basis, informed by patient symptoms at each clinical encounter and adjusted according to existing comorbidities.

The information in this section of the guideline is provided as an aid to monitoring adverse effects and is correct at the time of publication. Readers are advised to confirm the latest information with their pharmacy colleagues.

The NTM Drug Monograph section of the guideline is also available on the BTS website.

\section{AMIKACIN-INTRAVENOUS}

Please note amikacin is not licensed for the treatment of NTM in the UK.

\section{Dosage}

For intravenous administration only.

Amikacin is usually administered daily or three times per week.

- adults: $15 \mathrm{mg} / \mathrm{kg}$ once daily or $7.5 \mathrm{mg} / \mathrm{kg}$ twice daily or $15-25 \mathrm{mg} / \mathrm{kg}$ three times per week

- Obesity: It has been suggested that markedly obese patients should have an adjusted dose using ideal body weight plus $40 \%$ of the excess weight. The adjusted dose is due to the decreased distribution of extracellular fluids in adipose tissues.

- male ideal body weight $(\mathrm{kg})=50+(2.3 \times$ height in $\mathrm{cm}$ above 152.4$) / 2.54$

- female ideal body weight $(\mathrm{kg})=45.5+(2.3 \times$ height in $\mathrm{cm}$ above 152.4$) / 2.54$

Adjust dose and/or frequency according to serum amikacin concentration (see below).

- children: usually $15-30 \mathrm{mg} / \mathrm{kg}$ daily or $15-30 \mathrm{mg} / \mathrm{kg}$ three times per week

\section{PREPARATIONS}

- Parenteral: $100 \mathrm{mg} / 2 \mathrm{~mL}, 500 \mathrm{mg} / 2 \mathrm{~mL}$ injection.

\section{DRUG LEVEL MONITORING}

Indications for monitoring

- ensure therapeutic dose

- ensures that accumulation is not occurring in renal impairment.

\section{Target level}

- trough: $<5 \mathrm{mg} / \mathrm{L}$

- peak: $25-35 \mathrm{mg} / \mathrm{L}$ (daily dosing) or $65-80 \mathrm{mg} / \mathrm{L}$ (three times per week dosing).

Timing of sample

- Predose.

- Take a level 90-120 min and 6 hours after the infusion ends. Then plot on semilogarithmic paper and extrapolate back to time $=0$ and use this as the peak level.
- Alternatively, taking a level of 60 min after infusion ends may be appropriate as a measure of the peak level, but may underestimate the true peak level.

\section{Frequency of levels}

- peak serum level in the first week, repeat if poor response

- trough serum levels weekly for 4 weeks; this can reduce to fortnightly when stable.

\section{Suggested actions}

- trough level: high—extend interval

- peak level: high—reduce dose; low—increase dose.

\section{ADVERSE EFFECTS}

\section{Common}

- nephrotoxicity: accumulation if renal impairment

- ototoxicity: irreversible vestibulocochlear nerve damage.

Serious

- endocrine: hypocalcaemia, hypomagnesaemia and hypokalaemia

- neurological: neuromuscular blockade and respiratory paralysis (more common in neuromuscular disease; usually dose-related and self-limiting)

- audiological: ototoxicity-auditory > vestibular (higher with prolonged use and older age)

- renal: nephrotoxicity (higher with prolonged use).

\section{ADVERSE EFFECTS: MONITORING}

Renal auditory and vestibular monitoring is essential.

\section{Renal function}

Monitor twice weekly during month 1 , weekly during month 2, then fortnightly to the end of treatment. Consider increasing frequency of monitoring if there is evidence of renal impairment.

\section{Auditory and vestibular monitoring}

Loss of hearing usually occurs first and is detected by regular audiometric testing. Vertigo, loss of balance and auditory disturbances (tinnitus) are also signs of ototoxicity.

Ototoxicity on audiogram is defined as a $20 \mathrm{~dB}$ loss from baseline at any one test frequency or a $10 \mathrm{~dB}$ loss at any two adjacent test frequencies. If this occurs, amikacin should be discontinued or dosing reduced in frequency to avoid further hearing loss, although the hearing loss that has occurred is likely to be permanent. Expert advice should be sought at this point to consider a regimen change. We recommend that patients have baseline audiometry and then monthly audiometry until treatment with aminoglycoside ceases. A final audiometry review should be offered 2 months after the final dose.

Routine toxicity monitoring tests (FBC, U\&Es, LFTs) should be performed intermittently throughout antibiotic treatment for NTM-pulmonary disease (unless more specific advice is outlined above).

\section{Interactions}

- Diuretics: increased risk of ototoxicity if given with loop diuretics

- Bisphosphonates: increased risk of hypocalcaemia 
- Increased risk of nephrotoxicity if given with capreomycin, cephalosporins, ciclosporin, colistimethate sodium and tacrolimus

NB: There is no clinical benefit in prescribing amikacin AND capreomycin or kanamycin or streptomycin.

This information is not inclusive of all drug interactions. Please discuss with a pharmacist.

\section{CONTRAINDICATIONS AND CAUTIONS}

\section{Contraindications}

- Hypersensitivity: to amikacin or other aminoglycosides

- Myasthenia gravis: as amikacin may impair neuromuscular transmission

- Pregnancy: risk of vestibular or auditory nerve damage to fetus if used in second or third trimester.

\section{Cautions}

- Obese: Use ideal weight for height to calculate dose and monitor serum amikacin levels closely
- Elderly: Nephrotoxicity and ototoxicity common in the elderly; monitor and reduce dose if necessary

- Renal disease: use with caution; reduce the frequency of dosing and monitor serum concentrations.

\section{LABORATORY INFORMATION}

Please find up to date information at www.assayfinder.com regarding individual providers of drug level monitoring tests. Click on the provider for contact details. Turnaround time varies depending on the test and whether it is run locally or sent to an external lab. By contacting laboratories in advance, turnaround time can significantly be reduced.

Sample type: serum.

Volume required: $1-2 \mathrm{~mL}(\min 0.1 \mathrm{~mL})$.

Sample container: plain glass or plastic (non-SST).

Container type: any.

Availability: NS.

Turnaround time: telephoned same day if received 09:0015:00 Mon to Friday. Written confirmation report will be sent by first class post. 


\section{AMIKACIN-NEBULISED}

Please note amikacin is not licensed for the treatment of NTM in the UK.

\section{Dosage}

Adults and children: $500 \mathrm{mg}$ twice daily (nebulised). In cases of intolerance after an initial initiation period, a dose reduction may be considered to $250-500 \mathrm{mg}$ once or twice daily (seek expert advice).

In the UK, the injectable preparation is given via the nebulised route, using a suitable nebuliser with filter attachment (eg, SideStream Plus with filter attachment, or Pari LC Plus with filter attachment). The $250 \mathrm{mg} / \mathrm{mL}$ injection should be used and made up to $4 \mathrm{~mL}$ with sodium chloride $0.9 \%$.

Prior to administration the patient should receive a bronchodilator, for example, salbutamol, to reduce the risk of coughing and bronchospasm. This can be given nebulised or by their regular inhaler.

Amikacin for injection may only be mixed with sodium chloride $0.9 \%$. It must not be nebulised as a mixture with other nebulised drugs (eg, salbutamol, dornase alfa or other nebulised antibiotics).

\section{Preparations}

- Parenteral: $100 \mathrm{mg} / 2 \mathrm{~mL}, 500 \mathrm{mg} / 2 \mathrm{~mL}$ injection.

\section{DRUG LEVEL MONITORING}

A trough amikacin level is recommended 1 week after starting nebulised amikacin.

\section{Indications for monitoring}

- ensures that accumulation is not occurring in renal impairment.

\section{Target level}

trough: $<5 \mathrm{mg} / \mathrm{L}$.

Timing of sample

- predose.

\section{Frequency of levels}

trough serum levels, taken if required.

\section{Suggested actions}

trough level: high-extend interval.

\section{ADVERSE EFFECTS}

Systemic adverse effects are less frequent when amikacin is given via the nebulised route, than via the intravenous route. However, respiratory adverse effects may be more frequent.

\section{Common}

- respiratory: bronchospasm, dysphonia, sore throat, sore mouth, increased cough, wheeze and breathlessness

- nephrotoxicity: accumulation if renal impairment

- ototoxicity: irreversible vestibulocochlear nerve damage.

Serious

- audiological: ototoxicity-auditory > vestibular (higher with prolonged use and older age)

- endocrine: hypocalcaemia, hypomagnesaemia and hypokalaemia
- neurological: neuromuscular blockade and respiratory paralysis (more common in neuromuscular disease; usually dose-related and self-limiting)

- renal: nephrotoxicity (higher with prolonged use).

\section{ADVERSE EFFECTS: MONITORING}

Patients should have an initial supervised test dose with predose and postdose monitoring of lung function.

Renal, auditory and vestibular monitoring is essential.

Renal function

Monthly.

Consider increasing frequency of monitoring if there is evidence of renal impairment.

\section{Auditory and vestibular monitoring}

Loss of hearing usually occurs first and is detected by regular audiometric testing. Vertigo, loss of balance and auditory disturbances (tinnitus) are also signs of ototoxicity (higher risk with intravenous amikacin).

Ototoxicity on audiogram is defined as a $20 \mathrm{~dB}$ loss from baseline at any one test frequency or a $10 \mathrm{~dB}$ loss at any two adjacent test frequencies. If this occurs, amikacin should be discontinued or dosing reduced in frequency to avoid further hearing loss, although the hearing loss that has occurred is likely to be permanent. Expert advice should be sought at this point to consider a regimen change. We recommend that patients have baseline audiometry and then intermittently during treatment (frequency according to perceived risk and symptoms).

Routine toxicity monitoring tests (FBC, U\&Es, LFTs) should be performed intermittently throughout antibiotic treatment for NTM-pulmonary disease (unless more specific advice is outlined above).

\section{Interactions}

- diuretics: increased risk of ototoxicity if given with loop diuretics

- bisphosphonates: increased risk of hypocalcaemia

- increased risk of nephrotoxicity if given with capreomycin, cephalosporins, ciclosporin, colistimethate sodium and tacrolimus.

NB: There is no clinical benefit in prescribing amikacin AND capreomycin or kanamycin or streptomycin.

This information is not inclusive of all drug interactions. Please discuss with a pharmacist.

\section{CONTRAINDICATIONS AND CAUTIONS \\ Contraindications}

- hypersensitivity: to amikacin or other aminoglycosides

- myasthenia gravis: as amikacin may impair neuromuscular transmission

- pregnancy: risk of vestibular or auditory nerve damage to fetus if used in second or third trimester.

\section{Cautions}

- admixtures: amikacin for injection may only be mixed with sodium chloride $0.9 \%$; it must not be nebulised as a mixture with other nebulised drugs (eg, salbutamol, dornase alfa or other nebulised antibiotics)

- elderly: nephrotoxicity and ototoxicity common in the elderly; monitor and reduce dose if necessary

- renal disease: use with caution (higher risk with intravenous amikacin administration). 


\section{LABORATORY INFORMATION}

Please find up-to-date information at www.assayfinder.com regarding individual providers of drug level monitoring tests. Click on the provider for contact details. Turnaround time varies depending on the test and whether it is run locally or sent to an external lab. By contacting laboratories in advance, turnaround time can significantly be reduced.
Sample type: serum.

Volume required: $1-2 \mathrm{~mL}(\min 0.1 \mathrm{~mL})$.

Sample container: plain glass or plastic (non-SST).

Container type: any.

Availability: NS.

Turnaround time: telephoned same day if received 09:0015:00 Monday to Friday. Written confirmation report will be sent by first class post. 


\section{AZITHROMYCIN}

Please note azithromycin is not licensed for the treatment of NTM in the UK.

\section{Dosage}

Adults

250-500 mg once daily (oral) or $500 \mathrm{mg}$ three times per week (oral), depending on the NTM treatment regimen.

\section{Children}

Paediatric doses: (Note: Limited data on evidence for dosing in NTM. These doses are based on azithromycin dosing for respiratory tract infections in the latest BNF for children, September 2017 update.)

Child 6 months -17 years.

$10 \mathrm{mg} / \mathrm{kg}$ once daily (maximum per dose $500 \mathrm{mg}$ ) (oral).

Child 6 months-17 years (body weight 15-25 kg): $200 \mathrm{mg}$ once daily (oral).

Child 6 months-17 years (body weight 26-35 kg): $300 \mathrm{mg}$ once daily (oral).

Child 6 months-17 years (body weight $36-45 \mathrm{~kg}$ ): $400 \mathrm{mg}$ once daily (oral).

Child 6 months-17 years (body weight $46 \mathrm{~kg}$ and above): $500 \mathrm{mg}$ once daily (oral).

\section{Preparations}

- oral: $250 \mathrm{mg}$ capsules; $250 \mathrm{mg}$ tablets, $500 \mathrm{mg}$ tablets; $200 \mathrm{mg}$ in $5 \mathrm{~mL}$ suspension

- parenteral: $500 \mathrm{mg}$ powder for solution for infusion.

\section{DRUG LEVEL MONITORING}

Drug levels cannot currently be performed in the UK.

\section{ADVERSE EFFECTS}

Common

- dermatological: pruritus, rash

- gastrointestinal: abdominal pain, nausea, flatulence, vomiting, dyspepsia, anorexia

- general: fatigue, pain and inflammation on the local injection site

- investigations: raised eosinophils, reduced lymphocytes, reduced bicarbonate

- musculoskeletal: arthralgia

- neurological: dizziness, headache, paraesthesia, dysgeusia

- ophthalmic: visual impairment

- ototoxicity: deafness.

Serious

- cardiological: arrhythmia, prolonged QT interval, Torsades de Pointes, ventricular tachycardia

- dermatological: Stevens-Johnson syndrome

- haematological: leucopenia, neutropaenia, thrombocytopaenia, haemolytic anaemia

- hepatic: fulminant hepatitis, potentially leading to life-threatening liver failure

- immunological: angioedema, hypersensitivity, anaphylaxis

- infections: Pseudomembranous colitis

- neurological: myasthenia gravis

ototoxicity: tinnitus.

\section{ADVERSE EFFECTS: MONITORING}

ECG: baseline, 2 weeks and after the addition of any new medication that is known to prolong QT.

LFTs, U\&Es and FBC should be monitored sporadically throughout treatment. No specific frequency recommendations.

Audiometry: baseline and intermittently during treatment (frequency according to perceived risk and symptoms).

Routine toxicity monitoring tests (FBC, U\&Es, LFTs) should be performed intermittently throughout antibiotic treatment for NTM-pulmonary disease (unless more specific advice is outlined above).

\section{Interactions}

- amiodarone: increased risk of QT prolongation

- antacids: reduced absorption; take 2 hours apart

- antiemetics: increased risk of QT prolongation (eg, domperidone, metoclopramide, 5HT3 antagonists)

- ciclosporin: possible raised ciclosporin levels (monitor ciclosporin levels)

- colchicine: possible increased colchicine levels and increased toxicity (monitor for nausea, vomiting, diarrhoea, myopathy and pancytopaenia)

- digoxin: increased digoxin levels may occur (monitor digoxin levels)

- disopyramide: increased risk of QT prolongation

- ergot derivatives: ergotism has been precipitated by coadministration of some macrolide

- methadone: possible increased risk of QT prolongation

- statins: azithromycin is not usually expected to interact with statins due to lack of CYP3A4 inhibition, however case reports have been published

- tacrolimus: case reports of raised tacrolimus concentrations (monitor tacrolimus levels)

- warfarin: possible potentiated anticoagulation.

This information is not inclusive of all drug interactions. Please discuss with a pharmacist.

\section{CONTRAINDICATIONS AND CAUTIONS}

\section{Contraindications}

- hypersensitivity: to azithromycin or other macrolides.

\section{Cautions}

- cardiovascular disease: risk of prolonged QT interval in patients: (1) with congenital or documented QT prolongation; (2) concomitant administration of drugs known to prolong QT interval; 3) electrolyte disturbances; and (4) cardiac rhythm disorders

- liver disease: use with caution in significant hepatic disease

- myasthenia gravis: pregnancy and breast feeding

- renal disease: severe renal impairment.

\section{LABORATORY INFORMATION}

Please find up-to-date information at www.assayfinder.com regarding individual providers of drug level monitoring tests. Click on the provider for contact details. Turnaround time varies depending on the test and whether it is run locally or sent to an external lab. By contacting laboratories in advance, turnaround time can significantly be reduced. 


\section{BEDAQUILINE}

Please note bedaquiline is not licensed for the treatment of NTM in the UK.

There are limited data available on bedaquiline. Clinicians are advised to monitor patients closely to ensure the safe and effective use of this drug.

Patients should be advised that the following serious side effects can occur with bedaquiline: death, heart rhythm abnormalities and/or hepatitis. In addition, patients should also be advised about other potential side effects: nausea, joint pain, headache, increased blood amylase, haemoptysis, chest pain, anorexia and/ or rash. Additional testing may be needed to monitor or reduce the likelihood of adverse effects.

\section{Dosage}

Adults (aged 18-64 years): $400 \mathrm{mg}$ daily for the first 2 weeks, followed by $200 \mathrm{mg}$ three times per week for the remaining 22 weeks (maximum duration $=6$ months).

Children: not currently recommended in people aged less than 18 years.

Bedaquiline should be taken with food.

Patients should be advised to avoid alcohol while on bedaquiline.

There are no published case reports of the use of bedaquiline in children under 18 years for either the treatment of multidrug-resistant TB (MDR-TB) or non-tuberculous mycobacteria. Phase II clinical trials are currently evaluating the safety, tolerability, pharmacokinetics and antimycobacterial activity of bedaquiline in children and adolescents with MDR-TB, so no recommendations can currently be made. However there is anecdotal experience of using bedaquiline in children aged 12-18 years with MDR-TB at the same dose as adults.

\section{Preparations}

oral: $100 \mathrm{mg}$ tablets.

\section{DRUG LEVEL MONITORING}

- Drug levels need not be routinely measured.

\section{ADVERSE EFFECTS}

Report all suspected adverse drug reactions to the Medicines and Healthcare products Regulatory Agency through the Yellow Card Scheme.

\section{Common \\ - arthralgia \\ - chest pain \\ gastrointestinal: nausea \\ - neurological: headache \\ - respiratory: haemoptysis.}

\section{Serious}

- cardiovascular: QTc prolongation (more common in hypokalaemia, proarrhythmic conditions, in combination with other drugs that prolong the QT interval such as clofazimine, fluoroquinolones or macrolides)

- hepatic: increases in LFTs.

\section{ADVERSE EFFECTS: MONITORING}

ECG: baseline, 2 weeks, then every month and after the addition of any new medication that is known to prolong QT.
- Discontinue bedaquiline and all other QT prolonging drugs if the patient develops:

- clinically significant ventricular arrhythmia

- A QTc interval of >500 ms (confirmed by repeat ECG)

- Monitor ECGs frequently to confirm that the QTc interval has returned to baseline.

- If syncope occurs, obtain an ECG to detect QT prolongation. LFTs: at baseline, and repeated monthly.

U\&Es, calcium and magnesium: at baseline and repeated monthly and if QT prolongation is detected.

Routine toxicity monitoring tests (FBC, U\&Es, LFTs) should be performed intermittently throughout antibiotic treatment for NTM-pulmonary disease (unless more specific advice is outlined above).

\section{Interactions}

- antiarrhythmics: risk of prolonged QT interval (eg, amiodarone, sotalol, procainamide, disopyramide and quinidine)

- antiemetics: increased risk of QT prolongation (eg, domperidone, metoclopramide, 5HT3 antagonists)

- antiretrovirals: limited data

- antidepressants, tricyclic: risk of prolonged QT interval

- antipsychotics (thioridazine, haloperidol, chlorpromazine, trifluoperazine, percycline, prochlorperazine, fluphenazine, sertindole and pimozide): risk of prolonged QT interval

- azole antifungals (eg, ketoconazole, voriconazole, itraconazole, fluconazole): increased exposure to bedaquiline; avoid coadministration for more than 14 days

- carbamazepine: accelerated metabolism of bedaquiline resulting in reduced effect; avoid coadministration

- chloroquine and hydroxychloroquine: risk of prolonged QT interval

- clofazimine: risk of prolonged QT interval

- CYP3A4 inducers: accelerated metabolism of bedaquiline resulting in reduced effect; avoid coadministration

- CYP3A4 inhibitors: reduced metabolism resulting in increased serum concentrations of bedaquiline; avoid prolonged coadministration for more than 14 days

- fluoroquinolones: risk of prolonged QT interval.

- ivacaftor: possible increased serum levels of bedaquiline monitor for increased adverse effects.

- macrolides: risk of prolonged QT interval. Avoid co-administration for more than 14 days.

- Orkambi (lumacaftor/ivacaftor): accelerated metabolism of bedaquiline, avoid co-administration.

- phenytoin: accelerated metabolism of bedaquiline resulting in reduced effect. Avoid co-administration.

- rifampicin, rifabutin and rifapentine: accelerated metabolism of bedaquiline resulting in reduced effect; avoid coadministration

- statins: avoid coadministration.

This information is not inclusive of all drug interactions. Please refer to the SPC or BNF for further information, or discuss with a pharmacist.

\section{CONTRAINDICATIONS AND CAUTIONS}

\section{Contraindications}

- Pregnancy and breast feeding: There are no adequate or well-controlled studies in pregnant women. It is not known whether bedaquiline or its metabolites are excreted in human milk. 


\section{BTS guideline}

- Hypersensitivity: to bedaquiline.

- Children aged $<18$ years: The safety and effectiveness has not been established in children.

\section{Cautions}

Elderly patients $\geq 65$ years: lack of data in patients aged 65 and over to determine whether they respond differently from younger patients

Cardiovascular: Due to the risk of QT prolongation with bedaquiline, ECGs should be monitored closely in patients:

- taking other QT prolonging drugs (eg, fluoroquinolones, macrolides, clofazimine)

- with a history of Torsade de Pointes, congenital long QT syndrome, hypothyroidism and bradyarrhythmias, or uncompensated heart failure

- with serum calcium, magnesium or potassium levels below the lower limits of normal.
HIV/TB co-infection: limited or no information on the use of bedaquiline.

Alcohol or substance use: limited or no information on alcohol or substance use in association with bedaquiline; however, manufacturer recommends avoiding alcohol while taking bedaquiline.

Liver disease: lack of data in severe liver disease; no dose adjustment required in mild to moderate hepatic impairment.

Renal disease: Use with caution in patients with severe renal impairment or end-stage renal disease requiring haemodialysis or peritoneal dialysis.

\section{LABORATORY INFORMATION}

Please find up-to-date information at www.assayfinder.com regarding individual providers of drug level monitoring tests. Click on the provider for contact details. Turnaround time is usually a few days to a week, but this can be reduced by calling ahead and informing the laboratory in advance. 


\section{CEFOXITIN}

Please note cefoxitin is not licensed in the UK.

\section{Dosage}

Adults: $200 \mathrm{mg} / \mathrm{kg} /$ day in three divided doses (maximum $12 \mathrm{~g} /$ day) (intravenous infusion).

Children aged 1 month and above.

$150 \mathrm{mg} / \mathrm{kg} /$ day in 3-4 divided doses (maximum $12 \mathrm{~g} /$ day) (intravenous infusion).

Cefoxitin doses over $2 \mathrm{~g}$ should be given by intravenous infusion (diluted in sodium chloride $0.9 \%$ or glucose $5 \%$.

\section{Preparations}

- parenteral: $1 \mathrm{~g}, 2 \mathrm{~g}$ injection.

\section{DRUG LEVEL MONITORING}

- Drug levels cannot currently be performed in the UK.

\section{ADVERSE EFFECTS}

Common

- allergic: maculopapular rash, urticaria

- cardiovascular: hypotension

- haematological: leucopenia, thrombocytopaenia

- hepatic: transient increases in LFTs

- injection site reactions: thrombophlebitis or phlebitis

- investigations: interference with common assays (eg, Jaffé) measurements of creatinine concentrations, producing falsely high values.

\section{Serious}

- dermatological: urticaria, erythema multiforme, StevensJohnson syndrome

- haematological: leucopenia, neutropaenia, thrombocytopaenia, haemolytic anaemia, aplastic anaemia, haemorrhage

- hepatic: hepatic dysfunction including cholestasis, elevated bilirubin

- immunological: anaphylaxis

- infections: Pseudomembranous colitis
- neurological: seizures

- renal: acute renal failure, toxic nephropathy (rare).

\section{ADVERSE EFFECTS: MONITORING}

Routine toxicity monitoring tests (FBC, U\&Es, LFTs) should be performed intermittently throughout antibiotic treatment for NTM-pulmonary disease (unless more specific advice is outlined above).

\section{Interactions}

- aminoglycosides: increased risk of nephrotoxicity

- anticoagulants: increased INR with warfarin/pirfenidone

- furosemide: increased risk of nephrotoxicity

- probenecid: reduced renal excretion of cefoxitin, resulting in increased and prolonged serum levels.

This information is not inclusive of all drug interactions. Please discuss with a pharmacist.

\section{CONTRAINDICATIONS AND CAUTIONS}

\section{Contraindications}

- hypersensitivity: to cefoxitin sodium and other cephalosporins.

\section{Cautions}

- CNS disease: low CSF concentrations

- gastrointestinal disease: especially ulcerative colitis, regional enteritis or antibiotic-associated colitis

- hypersensitivity: to beta-lactam antibiotics: clinical and laboratory evidence of partial cross-allergenicity

- pregnancy and breast feeding

- Renal disease: reduce dose.

\section{LABORATORY INFORMATION}

Please find up-to-date information at www.assayfinder.com regarding individual providers of drug level monitoring tests. Click on the provider for contact details. Turnaround time varies depending on the test and whether it is run locally or sent to an external lab. By contacting laboratories in advance, turnaround time can significantly be reduced. 


\section{CIPROFLOXACIN}

Please note ciprofloxacin is not licensed for the treatment of NTM in the UK.

\section{Dosage}

Adults

Doses of 500-750 mg twice daily have been used (oral).

\section{Children}

Paediatric doses: (Note: Limited data on evidence for dosing in NTM. These doses are based on ciprofloxacin dosing for respiratory tract infections in the latest BNF for children, September 2017 update.)

Child: $20 \mathrm{mg} / \mathrm{kg}$ (max $750 \mathrm{mg}$ ) twice daily.

Neonate: $15 \mathrm{mg} / \mathrm{kg}$ twice daily.

\section{Preparations}

- oral: $250 \mathrm{mg}, 500 \mathrm{mg}, 750 \mathrm{mg}$ tablets; $250 \mathrm{mg} / 5 \mathrm{~mL}$ granules and solvent for oral suspension

- parenteral: $200 \mathrm{mg}, 400 \mathrm{mg}$ intravenous infusion.

\section{DRUG LEVEL MONITORING Indications for monitoring \\ - known or suspected malabsorption \\ - poor treatment response.}

\section{Target level}

The target serum levels for ciprofloxacin are uncertain.

For doses of $750 \mathrm{mg}$ twice daily, target levels have been suggested:

- trough level: $1.5 \mathrm{mg} / \mathrm{L}$

- peak level: $4.5 \mathrm{mg} / \mathrm{L}$.

For doses of $500 \mathrm{mg}$ twice daily, target levels have been suggested:

- trough level: $1 \mathrm{mg} / \mathrm{L}$

- peak level: $3 \mathrm{mg} / \mathrm{L}$.

\section{Timing of sample}

- 2 hours postoral dose (1 hour post intravenous administration)

- repeat at 6 hours if suspect delayed absorption

- trough levels_taken immediately prior to a dose.

\section{Frequency of levels}

- no need for regular monitoring.

\section{Suggested actions}

The appropriate action in response to low levels is uncertain. A level under $1 \mathrm{mg} / \mathrm{L}$, with poor clinical progression, may suggest inadequate dosing.

\section{ADVERSE EFFECTS}

\section{Common}

- administration: injection and infusion site reactions (only intravenous administration)

- dermatological: rash

- gastrointestinal: nausea, vomiting, diarrhoea, abdominal pain, dyspepsia.

\section{Serious}

- cardiovascular: QTc prolongation (rare)

- dermatological: photosensitivity, Stevens-Johnson syndrome or toxic epidermal necrolysis (rare)
- haematological: leucopenia, anaemia, neutropaenia, leucocytosis, thrombocytopaenia, thrombocythaemia, haemolytic anaemia, agranulocytosis, pancytopaenia (life-threatening), bone marrow depression (life-threatening)

- hepatic: hepatic impairment, hepatitis, liver necrosis (very rarely progressing to life-threatening hepatic failure)

- immunological: anaphylaxis

- infections: Pseudomembranous colitis

- metabolism: hyperglycaemia, hypoglycaemia

- musculoskeletal: exacerbation of myasthenia gravis, tendon inflammation and rupture (see contraindications below)

- neurological: seizures (caution in patients with CNS disorders)

- psychiatric: depression, psychosis

- renal: renal impairment (rare).

\section{ADVERSE EFFECTS: MONITORING}

ECG: baseline, 2 weeks and after the addition of any new medication that is known to prolong QT.

Blood glucose: should be monitored regularly in patients with diabetes (risk of hypoglycaemia).

Routine toxicity monitoring tests (FBC, U\&Es, LFTs) should be performed intermittently throughout antibiotic treatment for NTM-pulmonary disease (unless more specific advice is outlined above).

\section{Interactions}

- aluminium: reduced absorption of ciprofloxacin (take ciprofloxacin at least 1-2 hours before, or 4 hours after, aluminium-containing preparations)

- antacids: reduced absorption of ciprofloxacin (take ciprofloxacin at least 1-2 hours before, or 4 hours after, antacids)

- anticoagulants: enhanced anticoagulant effects of vitamin $\mathrm{K}$ antagonists (eg, warfarin, acenocoumarol, phenprocoumon or fluindione)

- antiemetics: increased risk of QT prolongation (eg, domperidone, metoclopramide, 5HT3 antagonists)

- calcium: reduced absorption of ciprofloxacin (take ciprofloxacin at least 1-2 hours before, or 4 hours after, calcium-containing preparations)

- ciclosporin: increased risk of nephropathy

- cinacalcet: possible increased cinacalcet levels (monitor parathyroid hormone and serum calcium levels)

- clozapine: increased clozapine serum levels (monitor for toxicity)

- dairy products or mineral-fortified drinks alone (eg, milk, yoghurt, calcium-fortified orange juice): reduced absorption of ciprofloxacin (avoid concurrent administration)

- drugs known to prolong the QT interval: use with caution in patients taking class IA and III antiarrhythmics, tricyclic antidepressants, macrolides, antipsychotics

- duloxetine: increased serum levels of duloxetine expected

- iron: reduced absorption of ciprofloxacin (take ciprofloxacin at least 1-2 hours before, or 4 hours after, iron-containing preparations)

- lidocaine: reduced clearance of intravenous lidocaine (usually well tolerated, but monitor for increased side effects)

- magnesium: reduced absorption of ciprofloxacin (take ciprofloxacin at least 1-2 hours before, or 4 hours after, magnesium-containing preparations)

- methotrexate: increased methotrexate serum concentrations (increased risk of toxicity) 
- mycophenolate: modest reduction in mycophenolate concentrations may occur (unlikely to be clinically important, but in transplant patients monitor for graft rejection)

- phenytoin: may result in increased or reduced serum levels of phenytoin (monitor serum phenytoin levels)

- phosphate binders (eg, sevelamer, lanthanum carbonate) reduced absorption of ciprofloxacin (take ciprofloxacin at least 1-2 hours before, or 4 hours after, phosphate binders)

- pirfenidone: increased pirfenidone levels (consider pirfenidone dose reduction)

- probenecid: reduced renal excretion of ciprofloxacin (increased serum levels)

- ropinirole: increased serum levels of ropinirole (monitor for increased side effects)

- sildenafil: increased sildenafil serum levels use with caution)

- theophylline: increased serum levels of theophylline leading to increased risk of convulsions; reduce dose of theophylline and monitor levels

- tizanidine: increased tizanidine serum concentrations (potentiates hypotensive and sedative effect).

This information is not inclusive of all drug interactions. Please discuss with a pharmacist.

\section{CONTRAINDICATIONS AND CAUTIONS}

\section{Contraindications}

- tizanidine: concomitant administration with tizanidine

- hypersensitivity: to sulfonamides, trimethoprim or co-trimoxazole.

\section{Cautions}

- cardiovascular disease: risk of prolonged QT interval in patients: (1) with congenital or documented QT prolongation; (2) concomitant administration of drugs known to prolong QT interval; (3) electrolyte disturbances; and (4) cardiac rhythm disorders

- children: use with caution in children (risk of arthropathy)

- CNS: history of seizures (lowered seizure threshold)

- diabetes: careful monitoring of blood glucose recommended due to risk of hypoglycaemia

- glucose-6-phosphate dehydrogenase deficiency

- myasthenia gravis

- renal disease (dose reduction required)

- sunlight: avoid direct exposure to either extensive sunlight or ultraviolet (UV) irradiation (photosensitivity risk)

- tendon disorders: history of tendon disease/disorder related to quinolone antibiotics.

\section{LABORATORY INFORMATION}

Please find up-to-date information at www.assayfinder.com regarding individual providers of drug level monitoring tests. Click on the provider for contact details. Turnaround time varies depending on the test and whether it is run locally or sent to an external lab. By contacting laboratories in advance, turnaround time can significantly be reduced.

Sample type: serum.

Volume required: $1-2 \mathrm{~mL}(\min 0.1 \mathrm{~mL})$.

Sample container: plain glass or plastic (non-SST).

Container type: any.

Availability: NS.

Turnaround time: telephoned same day if received 09:0015:00 Monday to Friday. Written confirmation report will be sent by first class post. 


\section{CLARITHROMYCIN}

Please note clarithromycin is not licensed for the treatment of NTM in the UK.

\section{Dosage}

For all patients over 12 years old.

By intravenous infusion

- $500 \mathrm{mg}$ twice a day given through a large proximal vein.

\section{By mouth}

- $500 \mathrm{mg}$ twice a day.

Paediatric doses: (Note: Limited data on evidence for dosing in NTM. These doses are based on clarithromycin dosing for respiratory tract infections in the latest BNF for children, December 2015 update.)

By intravenous infusion into large proximal vein

- child 1 month-11 years: $7.5 \mathrm{mg} / \mathrm{kg}$ (max $500 \mathrm{mg}$ ) twice a day

- child $12-18$ years: $500 \mathrm{mg}$ twice a day.

\section{By mouth}

Child 1 month -11 years

- body weight under $8 \mathrm{~kg}: 7.5 \mathrm{mg} / \mathrm{kg}$ twice a day

8-11 kg: $62.5 \mathrm{mg}$ twice a day

- 12-19kg: $125 \mathrm{mg}$ twice a day

- 20-29 kg: $187.5 \mathrm{mg}$ twice a day

- 30-40 kg:, $250 \mathrm{mg}$ twice a day.

Child $12-18$ years: $500 \mathrm{mg}$ twice a day

\section{Preparations}

oral: $250 \mathrm{mg}, 500 \mathrm{mg}$ tablets.

$125 \mathrm{mg} / 5 \mathrm{~mL}, 250 \mathrm{mg} / 5 \mathrm{~mL}$ suspension.

- parenteral: $500 \mathrm{mg}$ powder for solution for injection.

\section{DRUG LEVEL MONITORING}

- Drug levels need not be routinely measured.

\section{ADVERSE EFFECTS}

\section{Common}

- gastrointestinal: abdominal pain (2\%), diarrhoea (3\%-6\%), nausea $(3 \%)$, vomiting $(6 \%)$ and taste perversion $(3 \%-19 \%)$

- neurological: headache (2\%).

Serious

- cardiovascular: QTc prolongation (very rare)

- dermatological: (rare): anaphylaxis, leucocytoclastic vasculitis, toxic epidermal necrolysis and Stevens-Johnson syndrome

- hepatic: hepatomegaly, hepatic dysfunction and hepatic failure (rare)

- immunological: anaphylaxis

- infective: Clostridium difficile-associated diarrhoea and colitis

- ototoxicity: hearing loss and tinnitus reported in association with long-term use.

\section{ADVERSE EFFECTS: MONITORING}

- ECG: baseline, 2 weeks and after the addition of any new medication that is known to prolong QT

- audiometry: baseline and repeat if symptomatic

- routine toxicity monitoring tests (FBC, U\&Es, LFTs) should be performed intermittently throughout antibiotic treatment for NTM-pulmonary disease (unless more specific advice is outlined above).

\section{INTERACTIONS}

Use with caution with antivirals

- increased plasma concentrations of atazanavir, etravirine, nevirapine, telaprevir, tipranavir, and possibly maraviroc, rilpivirine

- increased clarithromycin concentrations with atazanavir, ritonavir, telaprevir, tipranavir

- reduced clarithromycin concentrations with etravirine, nevirapine

- increased risk of ventricular arrhythmias with saquinavir and telaprevir.

Increased plasma concentrations of

- antiepileptics: carbamazepine, phenytoin (monitor plasma concentrations)

- ciclosporin (avoid clarithromycin, or monitor ciclosporin plasma concentrations)

- coumarins, for example, warfarin (increased anticoagulant effect)

- ivabradine (avoid use)

- linezolid (consider drug level monitoring)

- rifabutin (requires rifabutin dose reduction)

- sirolimus (avoid clarithromycin, or monitor sirolimus plasma concentrations)

- statins (avoid use)

- tacrolimus (avoid clarithromycin, or monitor tacrolimus plasma concentrations)

- theophylline (reduce theophylline dose and monitor plasma concentrations)

- ticagrelor (avoid use).

- Orkambi (lumacaftor/ivacaftor): decreased plasma concentrations of clarithromycin. Consider switching to azithromycin. If Orkambi is initiated while a patient is taking clarithromycin, then the starting dose should be decreased to one tablet daily for the first week and then increased to two tablets twice daily thereafter as per SPC.

This information is not inclusive of all drug interactions. Please discuss with a pharmacist.

\section{CONTRAINDICATIONS AND CAUTIONS}

\section{Contraindications}

- hypersensitivity: to macrolides

- use of other drugs that may prolong the QT interval

- renal and liver disease: avoid in patients with both severe renal and liver disease.

\section{Cautions}

- pregnancy and breast feeding

- renal disease: use with caution; reduce the dose

- myasthenia gravis: macrolides may aggravate myasthenia gravis

- cardiovascular disease: due to the risk for QT prolongation, clarithromycin should be used with caution in patients with coronary artery disease, severe cardiac insufficiency, hypomagnesaemia, bradycardia ( $<50$ beats per minute) or when coadministered with other medicinal products associated with QT prolongation.

\section{LABORATORY INFORMATION}

Please find up-to-date information at www.assayfinder.com regarding individual providers of drug level monitoring tests. Click on the provider to discover contact details. Turnaround time varies depending on the test and whether it is run locally or sent to an external lab. By contacting laboratories in advance, turnaround time can significantly be reduced. 


\section{CLOFAZIMINE}

Please note clofazimine is not licensed in the UK.

\section{Dosage}

Adults: Recommend 100-200 mg once daily (oral).

Doses of $200 \mathrm{mg}$ daily for 2 months, then $100 \mathrm{mg}$ daily have been used. (Doses up to $300 \mathrm{mg}$ once daily have been used in leprosy.)

Children: Limited data, WHO recommendations for MDR-TB and XDR-TB are based on experience and expert opinion, and suggest $3-5 \mathrm{mg} / \mathrm{kg} /$ day (maximum $100 \mathrm{mg} /$ day). Close monitoring is recommended due to limited experience.

Clofazimine should be taken with meals or with milk to maximise absorption and reduce gastrointestinal adverse effects.

\section{Preparations}

- oral: $100 \mathrm{mg}$ capsules (unlicensed medicine).

\section{DRUG LEVEL MONITORING}

- Drug levels need not be routinely measured.

\section{ADVERSE EFFECTS}

\section{Common}

- dermatological: pink to brownish-black skin discolouration (resembling sun-tanning) within 1-4 weeks in 75\%-100\% of patients; it usually disappears within 6-12 months after stopping treatment, and it is important to advise patients of this prior to commencing treatment

- ichthyosis and dry skin (8\%-38\%), pruritus (5\%), rash (1\%-5\%), photosensitivity reactions (wear protective clothing and sunscreens)

- gastrointestinal: (up to 50\% of patients): abdominal pain, nausea, vomiting, diarrhoea and weight loss.

\section{Serious}

- gastrointestinal: (<1\%): bowel obstruction, GI haemorrhage

- ophthalmic: conjunctival pigmentation (38\%-57\%), subjective dimness of vision (12.3\%), and dry eyes, burning and other ocular irritation $(24.6 \%)$

- psychiatric: reactive depression due to skin discolouration

- other: splenic infarction, discolouration of body fluids.

\section{ADVERSE EFFECTS: MONITORING}

Risk of QT prolongation and ventricular tachyarrhythmias (thought to be Torsades de Pointes) has been highlighted in case reports

ECG: baseline, 2 weeks and after the addition of any new medication that is known to prolong QT.

Routine toxicity monitoring tests (FBC, U\&Es, LFTs) should be performed intermittently throughout antibiotic treatment for NTM-pulmonary disease (unless more specific advice is outlined above).

\section{Interactions}

- may reduce the absorption rate of rifampicin, but is unlikely to be clinically significant

- isoniazid may increase plasma and urinary concentrations of clofazimine and decrease skin concentrations

- Increased risk of prolonged QTc with other drugs that prolong QTc including fluoroquinolones and bedaquiline.

This information is not inclusive of all drug interactions. Please discuss with a pharmacist.

\section{CONTRAINDICATIONS AND CAUTIONS}

\section{Contraindications}

- hypersensitivity: to clofazimine

- hypersensitivity: to peanuts or soya, as clofazimine capsules contain soybean oil.

\section{Cautions}

- pregnancy and breast feeding

- renal disease: use with caution; dose reductions are not necessary

- liver disease: use with caution; metabolised by the liver, therefore may require dose adjustment in severe liver disease.

\section{LABORATORY INFORMATION}

Please find up-to-date information at www.assayfinder.com regarding individual providers of drug level monitoring tests. Click on the provider to discover contact details. Turnaround time varies depending on the test and whether it is run locally or sent to an external lab. By contacting laboratories in advance, turnaround time can significantly be reduced.

Not currently available in the UK. 


\section{COTRIMOXAZOLE (TRIMETHOPRIM/SULFAMETHOXAZOLE)}

Please note cotrimoxazole is not licensed for the treatment of NTM in the UK.

\section{Dosage}

Adults: $960 \mathrm{mg}$ twice daily (oral).

Adults: $1.44 \mathrm{~g}$ twice daily (intravenous).

Children: doses of $10-20 \mathrm{mg}$ of trimethoprim $/ \mathrm{kg} / \mathrm{dose}$ twice daily have been used (oral/intravenous).

\section{Preparations}

- oral: $480 \mathrm{mg}, 960 \mathrm{mg}$ tablets; $240 \mathrm{mg} / 5 \mathrm{~mL}, 480 \mathrm{mg} / 5 \mathrm{~mL}$ suspension

- parenteral: $480 \mathrm{mg} / 5 \mathrm{~mL}$ intravenous infusion.

\section{DRUG LEVEL MONITORING}

Indications for monitoring

$\checkmark$ renal impairment.

\section{Target level}

Trough levels:

- sulfamethoxazole: $<100 \mathrm{mg} / \mathrm{L}$

- trimethoprim: $5-7 \mathrm{mg} / \mathrm{L}$.

\section{Timing of sample}

- trough levels-taken immediately prior to a dose

- peak levels have been recommended for high dose co-trimoxazole as used in the treatment of PCP.

\section{Frequency of levels}

$\checkmark$ no need for regular monitoring.

\author{
ADVERSE EFFECTS \\ Common \\ - dermatological: rash \\ - gastrointestinal: nausea, vomiting, diarrhoea \\ - infections: Candida \\ - metabolic: hyperkalaemia \\ - neurological: headache.
}

\section{Serious}

- dermatological: photosensitivity, Stevens-Johnson syndrome or toxic epidermal necrolysis (rare)

- endocrine: hyponatraemia

- haematological: leucopenia, neutropaenia, thrombocytopaenia, agranulocytosis, megaloblastic anaemia, aplastic anaemia, haemolytic anaemia, methaemoglobinaemia, eosinophilia, purpura, haemolysis in certain susceptible G-6-PD-deficient patients

- hepatic: cholestatic jaundice, hepatic necrosis

- immunological: serum sickness, anaphylaxis, allergic myocarditis, angioedema, drug fever, allergic vasculitis, systemic lupus erythematosus

- infections: Pseudomembranous colitis

- neurological: aseptic meningitis, convulsions

- renal: renal impairment, interstitial nephritis

- respiratory: pulmonary infiltrates and respiratory hypersensitivity.

\section{ADVERSE EFFECTS: MONITORING}

U\&Es FBC, LFTs: monthly

Routine toxicity monitoring tests (FBC, U\&Es, LFTs) should be performed intermittently throughout antibiotic treatment for
NTM-pulmonary disease (unless more specific advice is outlined above).

\section{Interactions}

- anticoagulants: potentiation of the anticoagulant activity of warfarin

- ciclosporin: increased risk of deterioration in renal function

- clozapine: increased risk of bone marrow suppression

- contraceptives (oral): risk of contraceptive failure

- dapsone: possible increased risk of dapsone toxicity (methaemoglobinaemia)

- digoxin: trimethoprim may increase serum digoxin levels in some elderly people

- diuretics: (mainly thiazide): increased risk of thrombocytopaenia with or without purpura

- methotrexate: increased methotrexate levels and increased antifolate effects

- phenytoin: reduced metabolism (monitor serum phenytoin levels)

- pyrimethamine: increased risk of megaloblastic anaemia

- tacrolimus: possible additive neurotoxicity or nephrotoxicity

- zidovudine: increased risk of haematological adverse reactions (monitor FBC).

This information is not inclusive of all drug interactions. Please discuss with a pharmacist.

\section{CONTRAINDICATIONS AND CAUTIONS}

\section{Contraindications}

- breast feeding (where there is a risk of the infant developing hyperbilirubinaemia)

- hypersensitivity to sulfonamides, trimethoprim, or co-trimoxazole

- liver parenchymal damage

- megaloblastic anaemia due to folate deficiency

- pregnancy

- severe renal impairment.

Cautions

- skin: discontinue immediately if blood disorders or rash develops

- elderly patients (increased susceptibility to adverse effects)

- glucose-6-phosphate dehydrogenase deficiency

- people at risk of hyperkalaemia

- porphyria

- renal disease: reduce dose

- serious haematological disorders

- severe allergy

- severe asthma.

\section{LABORATORY INFORMATION}

Please find up-to-date information at www.assayfinder.com regarding individual providers of drug level monitoring tests. Click on the provider for contact details. Turnaround time varies depending on the test and whether it is run locally or sent to an external lab. By contacting laboratories in advance, turnaround time can significantly be reduced.

Sample type: serum.

Volume required: $1-2 \mathrm{~mL}(\min 0.1 \mathrm{~mL})$.

Sample container: plain glass or plastic (non-SST).

Container type: any.

Availability: NS.

Turnaround time: telephoned same day if received 09:0015:00 Monday to Friday. Written confirmation report will be sent by first class post. 


\section{DOXYCYCLINE}

Please note doxycycline is not licensed for the treatment of NTM in the UK.

\section{Dosage}

Adults and children (12-17 years): $100 \mathrm{mg}$ twice a day (oral). Take with a full glass of water to reduce risk of oesophagitis and oesophageal ulceration.

Paediatric doses: (Note: Limited data on evidence for dosing in NTM. These doses are based on doxycycline dosing for respiratory tract infections in the latest BNF for children, September 2017 update.)

\section{Preparations}

- oral: $50 \mathrm{mg}, 100 \mathrm{mg}$ capsules; $100 \mathrm{mg}$ dispersible tablets

- oral: $40 \mathrm{mg}$ modified release capsules (not recommended for use in NTM infections).

\section{DRUG LEVEL MONITORING} Indications for monitoring

- Drug levels need not be routinely measured.

\section{ADVERSE EFFECTS}

\section{Common}

- dermatological: photosensitivity, rash

- gastrointestinal: nausea, vomiting, diarrhoea, dysphagia.

\section{Serious}

- dermatological: exfoliative dermatitis, erythema multiforme, Steven-Johnson syndrome, toxic epidermal necrolysis, photosensitivity skin reactions, photo-onycholysis

- gastrointestinal: oesophagitis and oesophageal ulcerations (take doxycycline with plenty of water, during meals while sitting or standing)

- haematological: haemolytic anaemia, thrombocytopaenia, neutropaenia, porphyria and eosinophilia

- hepatotoxic: hepatitis, jaundice hepatic failure and pancreatitis

- immunological: anaphylaxis

- infections: Pseudomembranous colitis

- neurological: benign intracranial hypertension

- renal: renal dysfunction (lower risk with doxycycline than with other tetracyclines).

\section{ADVERSE EFFECTS: MONITORING}

Routine toxicity monitoring tests (FBC, U\&Es, LFTs) should be performed intermittently throughout antibiotic treatment for NTM-pulmonary disease (unless more specific advice is outlined above).

\section{Interactions}

- alcohol: reduced doxycycline serum levels in alcoholic patients, but not in moderate or even occasional heavy drinking patients (consider double-dose doxycycline)

- aluminium: reduced absorption of doxycycline (take doxycycline at least 2-3 hours before or after aluminium-containing preparations)
- antacids: reduced absorption of doxycycline (take doxycycline at least 2-3 hours before or after antacids)

- anticoagulants: prolonged prothrombin time in patients taking warfarin (monitor INR and reduce warfarin dose)

- antiepileptics: barbiturates, phenytoin or carbamazepine may reduce serum doxycycline level (consider double-dose doxycycline)

- calcium: reduced absorption of doxycycline (take doxycycline at least 2-3 hours before or after calcium-containing preparations)

- ciclosporin: increased plasma concentration of ciclosporin (monitor closely)

- contraceptives: reduced effect of oral contraceptives (loss of effect, breakthrough bleeding)

- ergot alkaloids: increased risk of ergotism

- iron: reduced absorption of doxycycline (take doxycycline at least 2-3 hours before or iron-containing preparations)

- lanthanum carbonate: reduced absorption of doxycycline (take doxycycline at least 2-3 hours before or after lanthanum)

- lithium: case reports of increased serum lithium levels (monitor levels)

- magnesium: reduced absorption of doxycycline (take doxycycline at least 2-3 hours before or after magnesium-containing preparations)

- retinoids: (eg, acitretin, isotretinoin): risk of pseudotumour cerebri (benign intracranial hypertension); avoid use

- rifampicin: reduced serum doxycycline level (avoid, or increase dose).

This information is not inclusive of all drug interactions. Please discuss with a pharmacist.

\section{CONTRAINDICATIONS AND CAUTIONS}

\section{Contraindications}

- hypersensitivity: to doxycycline or to other tetracyclines

- children: up to the age of 12 years (due to risks of permanent teeth discolouration and enamel hypoplasia)

- pregnancy and breast feeding.

Cautions

- hepatic impairment, and patients using other potentially hepatotoxic drugs

- myasthenia gravis (potential for weak neuromuscular blockade)

- porphyria

- sunlight: avoid direct exposure to either extensive sunlight or UV irradiation (photosensitivity risk)

- systemic lupus erythematosus (risk of exacerbation).

\section{LABORATORY INFORMATION}

Please find up-to-date information at www.assayfinder.com regarding individual providers of drug level monitoring tests. Click on the provider for contact details. Turnaround time varies depending on the test and whether it is run locally or sent to an external lab. By contacting laboratories in advance, turnaround time can significantly be reduced. 


\section{BTS guideline}

\section{ETHAMBUTOL}

Please note ethambutol is not licensed for the treatment of NTM in the UK.

\section{Dosage}

Adults: $15 \mathrm{mg} / \mathrm{kg}$ once daily (oral) or $25 \mathrm{mg} / \mathrm{kg}$ three times per week (oral), depending on the NTM treatment regimen. (Round the dose up or down to the closest whole number of tablets.)

Note: Ethambutol should be dosed on lean body weight.

Male ideal body weight $(\mathrm{kg})=50+(2.3 \times$ height in $\mathrm{cm}$ above 152.4)/2.54.

Female ideal body weight $(\mathrm{kg})=45.5+(2.3 \times$ height in $\mathrm{cm}$ above 152.4)/2.54.

Children ( 1 month to 18 years): $20 \mathrm{mg} / \mathrm{kg}$ once daily (oral) (Doses should be rounded down to facilitate administration of suitable volumes of liquid or an appropriate strength of tablet.)

\section{Preparations}

- oral: $100 \mathrm{mg}, 400 \mathrm{mg}$ tablets

- suspension (as a manufactured 'special'-unlicensed medicine)

- an intravenous preparation may be available from specialist importers.

\section{DRUG LEVEL MONITORING}

Indications for monitoring

- known or suspected malabsorption

- renal impairment

- poor treatment response.

Target level: 2-6 mg/L (peak).

\section{Timing of sample}

- 2 hours postdose

- repeat at 6 hours if suspect delayed.

\section{Frequency of levels}

- Drug levels need not be routinely measured.

\section{ADVERSE EFFECTS}

\section{Common}

- endocrine: hyperuricaemia

- gastrointestinal: nausea, vomiting.

\section{Serious}

- ophthalmic: optic neuritis $(1 \%-6 \%$; greatest risk at doses $>25 \mathrm{mg} / \mathrm{kg} / \mathrm{day}$, or $>2$ months treatment), red/green colour blindness.

\section{ADVERSE EFFECTS: MONITORING}

Ophthalmic: Visual acuity and colour discrimination testing at baseline and if symptoms are reported.

Routine toxicity monitoring tests (FBC, U\&Es, LFTs) should be performed intermittently throughout antibiotic treatment for NTM-pulmonary disease (unless more specific advice is outlined above).

\section{Interactions}

- isoniazid: possible increased risk of optic neuropathy caused by ethambutol.

This information is not inclusive of all drug interactions. Please discuss with a pharmacist.

\section{CONTRAINDICATIONS AND CAUTIONS}

Contraindications

- hypersensitivity: to ethambutol

- ophthalmic: optic neuritis and poor vision unless clinical judgement determines that it may be used.

\section{Cautions}

- renal disease: reduce dose in severe renal impairment

- young children: due to difficulty in testing eyesight and obtaining reports on symptomatic visual changes

- elderly patients: due to the risks of ophthalmic adverse effects.

\section{LABORATORY INFORMATION}

Please find up-to-date information at www.assayfinder.com regarding individual providers of drug level monitoring tests. Click on the provider to discover contact details. Turnaround time varies depending on the test and whether it is run locally or sent to an external lab. By contacting laboratories in advance, turnaround time can significantly be reduced.

Sample type: serum.

Volume required: $2 \mathrm{~mL}$.

Sample container: plain (non-SST).

Container type: any.

Availability: office hours.

Turnaround time: 7 days. 


\section{IMIPENEM}

Please note imipenem is not licensed for the treatment of NTM in the UK.

\section{Dosage}

(Note: Limited data on evidence for dosing in NTM.)

Adults (>50 kg): $1 \mathrm{~g}$ twice a day (intravenous). Doses of $750 \mathrm{mg}$ three times daily (intravenous) have been used.

Adults (<50 kg): $15 \mathrm{mg} / \mathrm{kg}$ twice a day (intravenous).

Children: $20-40 \mathrm{mg} / \mathrm{kg}(\max 2 \mathrm{~g})$ three times a day (intravenous).

In renal failure dose reduction may be necessary. Please discuss with a pharmacist.

\section{Preparations}

- parenteral: 500/500 mg powder for solution for infusion.

\section{DRUG LEVEL MONITORING}

- Drug levels need not be routinely measured.

\section{ADVERSE EFFECTS}

\section{Common}

- dermatological: rash and urticaria (3\%), injection site pain

- gastrointestinal: nausea, vomiting, diarrhoea

- haematological: thrombophlebitis (3\%), eosinophilia (4\%)

- hepatic: transient mild increases in LFTs

- Renal: transient increases in urea and/or serum creatinine concentrations $(<2 \%)$.

\section{Serious}

- immunological: anaphylaxis

- infections: Clostridium difficile-associated diarrhoea and colitis

- haematological: pancytopaenia, neutropaenia, leucopenia, thrombocytopaenia, thrombocytosis (rare): agranulocytosis

- neurological: seizures
- renal (rare): acute renal failure, oliguria/anuria, polyuria, urine discolouration.

\section{ADVERSE EFFECTS: MONITORING}

Routine toxicity monitoring tests (FBC, U\&Es, LFTs) should be performed intermittently throughout antibiotic treatment for NTM-pulmonary disease (unless more specific advice is outlined above).

\section{Interactions}

- ganciclovir: increased risk of convulsions

- valproate: reduced serum concentrations of valproate; avoid concomitant use.

This information is not inclusive of all drug interactions. Please discuss with a pharmacist.

\section{CONTRAINDICATIONS AND CAUTIONS}

Contraindications

- hypersensitivity: severe hypersensitivity to penicillins, carbapenems or cephalosporins

- pregnancy.

\section{Cautions}

- breast feeding

- renal impairment: increased risk of seizures, reduce dose

- liver disease: monitor LFTs (risk of increase in transaminases, hepatic failure and fulminant hepatitis).

\section{LABORATORY INFORMATION}

Please find up-to-date information at www.assayfinder.com regarding individual providers of drug level monitoring tests. Click on the provider to discover contact details. Turnaround time for tests is usually a few days to a week, but this can be reduced by calling ahead and informing the laboratory in advance. 


\section{ISONIAZID}

Please note isoniazid is not licensed for the treatment of NTM in the UK.

\section{Dosage}

Adults: $300 \mathrm{mg}$ once a day (oral or intravenous). Consider $5 \mathrm{mg} /$ $\mathrm{kg}$ once a day if low body weight (oral or intravenous).

Children: $10 \mathrm{mg} / \mathrm{kg}$ (max $300 \mathrm{mg}$ ) once a day (oral or intravenous).

Doses should be rounded up to facilitate administration of suitable volumes of liquid or an appropriate strength of tablet.

Isoniazid should be taken $30-60 \mathrm{~min}$ before food, or 2 hours after food.

Pyridoxine can be used to reduce the risk of peripheral neuropathy in all patients taking isoniazid. In particular it should be prescribed for those most at-risk, such as patients with diabetes, alcohol abuse or malnutrition.

\section{Preparations}

- oral: $100 \mathrm{mg}$ capsules

- liquid (as a manufactured 'special'-unlicensed medicine)

- Rifinah 300/150 tablets (rifampicin 300 mg, isoniazid $150 \mathrm{mg})$

- Rifinah 150/100 tablets (rifampicin 150 mg, isoniazid $100 \mathrm{mg}$ )

- parenteral: $50 \mathrm{mg} / 2 \mathrm{~mL}$ ampoules.

\section{DRUG LEVEL MONITORING \\ Indications for monitoring}

- known or suspected malabsorption

- poor treatment response.

Target level: $3-5 \mathrm{mg} / \mathrm{mL}$ (peak).

Timing of sample

- 2 hours postdose

- repeat at 6 hours if suspect delayed absorption.

\section{Frequency of levels}

- Drug levels need not be routinely measured.

\section{Adherence monitoring}

INH strips can be used to measure adherence to isoniazid treatment.

- BBL Taxo INH Test Strips are absorbent paper strips that are in colour green, blue or purple in the presence of isonicotinic acid (a metabolite of isoniazid).

- BBL Taxo INH Test Control is an isoniazid-impregnated disc that will yield a positive result in the test procedure.

\section{ADVERSE EFFECTS \\ Common \\ - neurological: peripheral neuropathy \\ - hepatic: transient increases in LFTs.}

Serious

- dermatological: skin reactions, for example, urticaria (uncommon)
- haematological: agranulocytosis, megaloblastic anaemia, thrombocytopaenia

- hepatic: hepatotoxicity (rare)

- immunological: drug-induced lupus (rare)

- musculoskeletal: arthralgia, rhabdomyolysis

- neurological: seizure, psychosis (rare).

\section{ADVERSE EFFECTS: MONITORING}

Routine toxicity monitoring tests (FBC, U\&Es, LFTs) should be performed intermittently throughout antibiotic treatment for NTM-pulmonary disease (unless more specific advice is outlined above).

\section{Interactions}

- Carbamazepine: increased plasma concentration of carbamazepine; increased risk of hepatotoxicity.

- Food: reduced absorption. Take isoniazid 30-60 min before food, or 2 hours after food.

- Food: Possible increased risk of headache, sweating, palpitations, flushing, hypotension when eating certain foods such as cheese, skipjack tuna or other tropical fish, or red wine. Usually, no dietary restrictions are required unless symptoms are experienced. This reaction is thought to be due to the high histamine or tyramine content of these foods and drink, resulting in an exaggerated histamine poisoning reaction due to inhibition of histamine metabolism by isoniazid, or the sympathomimetic action of tyramine due to inhibition of monoamine oxidase by isoniazid.

- Ivacaftor: increased plasma concentration of ivacaftor (avoid concomitant use with isoniazid).

This information is not inclusive of all drug interactions. Please discuss with a pharmacist.

\section{CONTRAINDICATIONS AND CAUTIONS}

Contraindications

- hypersensitivity: to isoniazid.

Cautions

- liver disease, alcohol abuse, hepatitis B coinfection: monitor LFTs closely

- malnutrition, HIV coinfection, diabetes mellitus and alcohol dependence: increased risk of peripheral neuropathy; prescribe prophylactic pyridoxine.

\section{LABORATORY INFORMATION}

Please find up-to-date information at www.assayfinder.com regarding individual providers of drug level monitoring tests. Click on the provider to discover contact details. Turnaround time varies depending on the test and whether it is run locally or sent to an external lab. By contacting laboratories in advance, turnaround time can significantly be reduced.

Sample type: plasma.

Volume required: $2 \mathrm{~mL}$.

Sample container: fluoride oxalate.

Container type: any.

Availability: office hours.

Turnaround time: 7 days. 


\section{LINEZOLID}

Please note linezolid is not licensed for the treatment of NTM in the UK.

\section{Dosage}

By mouth or intravenous infusion.

Adults, and adolescents: $600 \mathrm{mg}$ once a day or twice a day (oral or intravenous). Consider reducing to $300 \mathrm{mg}$ once daily if serious adverse effects develop.

Children (age 1 week - 12 years):

Based on WHO recommendations for drug-resistant TB:

- children age 1 week to 9 years: $10 \mathrm{mg} / \mathrm{kg}$ twice a day (maximum $600 \mathrm{mg} /$ day)

- children age $>10$ years: $10 \mathrm{mg} / \mathrm{kg}$ once a day (maximum $600 \mathrm{mg} /$ day). Doses of $300 \mathrm{mg}$ once daily have been used.

\section{PREPARATIONS}

oral: $600 \mathrm{mg}$ tablets.

$100 \mathrm{mg} / 5 \mathrm{~mL}$ granules for oral suspension

- parenteral: $600 \mathrm{mg} / 300 \mathrm{~mL}$ solution for infusion.

\section{DRUG LEVEL MONITORING \\ Indications for monitoring \\ - known or suspected malabsorption \\ - poor treatment response.}

Target level: $12-24 \mathrm{mg} / \mathrm{L}$ (peak).

\section{Timing of sample}

- 2 hours postoral dose or 1 hour post intravenous infusion.

\section{Frequency of levels}

- no need for regular monitoring.

\author{
ADVERSE EFFECTS \\ Common \\ - gastrointestinal: diarrhoea (4\%), nausea (3\%), vomiting \\ - neurological: headache $(2 \%)$ \\ - infections: Candidiasis, particularly oral and vaginal (1\%) \\ - hepatic: transient increases in LFTs.
}

\section{Serious}

- metabolic: lactic acidosis

- dermatological: urticaria, rash; (rare): bullous disorders such as Stevens-Johnson syndrome and toxic epidermal necrolysis

- haematological: myelosuppression

- neurological: peripheral neuropathy, seizure, serotonin syndrome

- ophthalmic: optic neuropathy-increased risk with prolonged treatment.

\section{ADVERSE EFFECTS: MONITORING}

FBC: Weekly for the 2 months. Consider reducing to monthly if stable thereafter.

Note: Some papers in MDR-TB/XDR-TB suggest using a lower dose of $300 \mathrm{mg}$ may be better tolerated in terms of myelosuppression.

\section{Visual acuity and colour discrimination}

Encourage patients to report any changes to their vision and consider performing visual acuity and colour discrimination testing (Snellen and Ishihara charts) monthly. Refer to ophthalmology if any changes.

\section{Peripheral neuropathy}

Encourage patients to report any symptoms suggestive of peripheral neuropathy and arrange nerve conduction studies should these arise.

Routine toxicity monitoring tests (FBC, U\&Es, LFTs) should be performed intermittently throughout antibiotic treatment for NTM-pulmonary disease (unless more specific advice is outlined above).

\section{Interactions}

- Clarithromycin: increases linezolid serum levels with risk of toxicity (consider drug level monitoring).

- Patients should avoid consuming large amounts of tyraminerich foods (such as mature cheese, yeast extracts, undistilled alcoholic beverages and fermented soya bean products). In addition, linezolid should not be given with another MAOI or within 2 weeks of stopping another MAOI. Unless close observation and blood pressure monitoring is possible, avoid in those receiving SSRIs, 5HT1 agonists ('triptans'), tricyclic antidepressants, sympathomimetics, dopaminergics, buspirone, pethidine and possibly other opioid analgesics.

This information is not inclusive of all drug interactions. Please discuss with a pharmacist.

\section{CONTRAINDICATIONS AND CAUTIONS \\ Contraindications}

- Hypersensitivity: to linezolid.

- Monoamine oxidase inhibitors: Avoid concomitant use of other drugs that inhibit monoamine oxidases A or B (eg, phenelzine, isocarboxazid, selegiline, moclobemide) or within 2 weeks of taking any such medicinal product.

- Avoid in patients with uncontrolled hypertension, phaeochromocytoma, carcinoid tumour, thyrotoxicosis, bipolar depression, schizophrenia or acute confusional states.

- Breast feeding.

\section{Cautions}

- Pregnancy.

- Avoid consumption of large amounts of tyramine-rich foods.

- Epilepsy/history of seizures: increased risk of convulsions.

- Renal impairment: No dose adjustment is required. However two primary metabolites may accumulate in severe renal impairment, but the clinical significance of this is unknown. Use with caution and monitor for adverse effects closely (see above).

- Liver disease: No dose adjustment is required. However due to limited clinical data, use with caution and monitor for adverse effects closely (see above).

- Peripheral and optic neuropathy: Patients should be advised to report symptoms of visual impairment.

\section{LABORATORY INFORMATION}

Please find up-to-date information at www.assayfinder.com regarding individual providers of drug level monitoring tests. Click on the provider to discover contact details. Turnaround 


\section{BTS guideline}

time varies depending on the test and whether it is run locally or sent to an external lab. By contacting laboratories in advance, turnaround time can significantly be reduced.

Sample type: serum.

Volume required: $2 \mathrm{~mL}$ (min $0.1 \mathrm{~mL}$ ).

Sample container: plain plastic (non-SST).

Container type: any.
Availability: NS.

Turnaround time: telephoned same day if received 09:0015:00 Monday to Friday. Written confirmation report will be sent by first class post.

The sample must be heat-treated before dispatch if HIV-positive.

Please telephone at least 1 day in advance of the sample. 


\section{MINOCYCLINE}

Please note minocycline is not licensed for the treatment of NTM in the UK.

\section{Dosage}

Adults: $100 \mathrm{mg}$ twice daily (oral).

Children (12-17years): $100 \mathrm{mg}$ twice daily (oral).

Paediatric doses: (Note: Limited data on evidence for dosing in NTM. These doses are based on minocycline dosing for respiratory tract infections in the latest BNF for children, September 2017 update.)

\section{Preparations}

- oral: $50 \mathrm{mg}, 100 \mathrm{mg}$ capsules, $50 \mathrm{mg}, 100 \mathrm{mg}$ tablets, $100 \mathrm{mg}$ modified release capsules.

\section{DRUG LEVEL MONITORING}

Indications for monitoring

- Drug levels need not be routinely measured.

\section{ADVERSE EFFECTS}

Common

- there may be a higher incidence of adverse effects of minocycline than for doxycycline

- dermatological: photosensitivity, rash

- gastrointestinal: nausea, vomiting, diarrhoea, dysphagia

- neurological: dizziness, headache.

Serious

- cardiac: pericarditis (rare)

- dermatological: exfoliative dermatitis, erythema multiforme, Steven-Johnson syndrome, toxic epidermal necrolysis, photosensitivity skin reactions, photo-onycholysis, skin hyperpigmentation

- gastrointestinal: oesophagitis and oesophageal ulcerations (take minocycline with plenty of water, during meals while sitting or standing)

- haematological: haemolytic anaemia, thrombocytopaenia, neutropaenia, eosinophilia

- hepatotoxic: hepatitis, jaundice hepatic failure and pancreatitis

- immunological: anaphylaxis, lupus-like syndrome

- infections: Pseudomembranous colitis

- neurological: benign intracranial hypertension

- renal: renal dysfunction (lower risk with doxycycline than with other tetracyclines)

- respiratory: pulmonary infiltration, pulmonary eosinophilia.

\section{ADVERSE EFFECTS: MONITORING}

Routine toxicity monitoring tests (FBC, U\&Es, LFTs) should be performed intermittently throughout antibiotic treatment for
NTM-pulmonary disease (unless more specific advice is outlined above).

\section{Interactions}

- aluminium: reduced absorption of minocycline (take minocycline at least 2-3 hours before or after aluminium-containing preparations)

- anticoagulants: prolonged prothrombin time in patients taking warfarin (monitor INR and reduce warfarin dose)

- calcium: reduced absorption of minocycline (take minocycline at least 2-3 hours before or after calcium-containing preparations)

- contraceptives: reduced effect of oral contraceptives (loss of effect, breakthrough bleeding)

- iron: reduced absorption of minocycline (take minocycline at least 2-3 hours before or iron-containing preparations)

- lithium: case reports of increased serum lithium levels (monitor levels)

- magnesium: reduced absorption of minocycline (take minocycline at least 2-3 hours before or after magnesium-containing preparations)

- retinoids: (eg, acitretin, isotretinoin): risk of pseudotumour cerebri (benign intracranial hypertension); avoid use

- zinc: reduced absorption of minocycline (take minocycline at least 2-3 hours before or after zinc-containing preparations).

This information is not inclusive of all drug interactions. Please discuss with a pharmacist.

\section{CONTRAINDICATIONS AND CAUTIONS}

Contraindications

- hypersensitivity to minocycline, or to other tetracyclines

- children up to the age of 12 years (due to risks of permanent teeth discolouration and enamel hypoplasia)

- pregnancy and breast feeding

- systemic lupus erythematosus (risk of exacerbation).

Cautions

- sunlight: avoid direct exposure to either extensive sunlight or UV irradiation (photosensitivity risk)

- hepatic impairment and patients using other potentially hepatotoxic drugs

- myasthenia gravis (potential for weak neuromuscular blockade)

- oral contraceptives: risk of contraceptive failure if diarrhoea or breakthrough bleeding occurs.

\section{LABORATORY INFORMATION}

Please find up-to-date information at www.assayfinder.com regarding individual providers of drug level monitoring tests. Click on the provider for contact details. Turnaround time varies depending on the test and whether it is run locally or sent to an external lab. By contacting laboratories in advance, turnaround time can significantly be reduced. 


\section{MOXIFLOXACIN}

Please note moxifloxacin is not licensed to treat NTM in the UK.

\section{Dosage}

Adults: $400 \mathrm{mg}$ once a day (oral or intravenous).

Children: $7.5-10 \mathrm{mg} / \mathrm{kg}$ once a day (oral).

\section{Preparations}

oral: $400 \mathrm{mg}$ tablets

- parenteral: $400 \mathrm{mg} / 250 \mathrm{~mL}$ solution for infusion.

\section{DRUG LEVEL MONITORING}

Indications for monitoring

- known or suspected malabsorption

- poor treatment response.

Target level: $2.5-4 \mathrm{mg} / \mathrm{L}$ (peak).

\section{Timing of sample}

- 2 hours postdose

- repeat at 6 hours if suspect delayed absorption.

\section{Frequency of levels}

- no need for regular monitoring.

\section{ADVERSE EFFECTS}

\section{Common}

- cardiovascular: QTc prolongation (more common in hypokalaemia, proarrhythmic conditions, in combination with other drugs that prolong the QT interval such as ondansetron)

- gastrointestinal: nausea, vomiting, diarrhoea

- hepatic: transient increases in LFTs

- other: dizziness, headache.

Serious

- cardiovascular: QTc prolongation (rare; more common in hypokalaemia, and predisposing cardiac conditions)

- dermatological: Stevens-Johnson syndrome or toxic epidermal necrolysis (rare)

- haematological: (uncommon) agranulocytosis, aplastic anaemia, haemolytic anaemia, thrombocytopaenia

- hepatic: acute hepatitis (rare)

- immunological: anaphylaxis, immune hypersensitivity (uncommon)

- metabolic: hypoglycaemia (in patients on hypoglycaemic drugs, uncommon)

- musculoskeletal: tendon inflammation and rupture (see contraindications below)

- neurological: seizures (caution in patients with CNS disorders)

- renal: renal impairment (rare)

- respiratory: extrinsic allergic alveolitis (rare)

other: serum sickness (rare).

\section{ADVERSE EFFECTS: MONITORING}

ECG: baseline, 2 weeks and after the addition of any new medication that is known to prolong QT.

Blood glucose should be monitored regularly in patients with diabetes (risk of hypoglycaemia).

Routine toxicity monitoring tests (FBC, U\&Es, LFTs) should be performed intermittently throughout antibiotic treatment for NTM-pulmonary disease (unless more specific advice is outlined above).

\section{Interactions}

- antacids: reduced absorption of moxifloxacin

- antiarrhythmics: increased risk of ventricular arrhythmias with amiodarone or disopyramide

- antiemetics: increased risk of QT prolongation (eg, domperidone, metoclopramide, 5HT3 antagonists)

- antidepressants: increased risk of ventricular arrhythmias with tricyclics

- antimalarials: increased risk of ventricular arrhythmias with chloroquine, hydroxychloroquine, mefloquine, quinine

- antipsychotics: increased risk of ventricular arrhythmias with benperidol, droperidol, haloperidol, phenothiazines, pimozide and zuclopenthixol

- antivirals: increased risk of ventricular arrhythmias with saquinavir

- beta-blockers: increased risk of ventricular arrhythmias with sotalol

- ciclosporin: increased risk of nephropathy

- erythromycin: increased risk of ventricular arrhythmias when erythromycin given via intravenous route

- iron: reduced absorption of moxifloxacin

- NSAIDs: possible increased risk of convulsions

- pentamidine: increased risk of ventricular arrhythmias

- theophylline: increased risk of convulsions; reduce dose of theophylline and monitor levels

- zinc: reduced absorption of moxifloxacin

- drugs known to prolong the QT interval: use with caution in patients taking class IA and III antiarrhythmics, tricyclic antidepressants, macrolides, antipsychotics.

This information is not inclusive of all drug interactions. Please discuss with a pharmacist.

\section{CONTRAINDICATIONS AND CAUTIONS}

Contraindications

- Hypersensitivity: to moxifloxacin or other quinolones.

- Tendon damage: rarely reported but damage or rupture may occur within 48 hours of treatment and several months after stopping treatment. Increased risk in patients with a history of tendon disorders related to quinolone use, aged over 60 years, concomitant use of corticosteroids. Cease all quinolone treatment if tendinitis suspected.

- Pregnancy: avoid in pregnancy, animal studies have shown quinolones cause arthropathy.

- Breast feeding: avoid, present in milk in animal studies.

- Children: Moxifloxacin is contraindicated in the UK for use in children or growing adolescents. Use in TB with caution. Arthropathy has developed in weight-bearing joints in young animals.

- Cardiovascular: due to the risk of QT prolongation with moxifloxacin, it should not be used in patients with congenital or documented acquired QT prolongation, clinically relevant bradycardia, clinically relevant heart failure with reduced left ventricular ejection fraction, history of symptomatic arrhythmias, or electrolyte disturbances, particularly in uncorrected hypokalaemia.

- Liver disease: chronic liver disease, particularly Child-Pugh severity score $\mathrm{C}$ and in those patients with transaminase levels fivefold greater than the upper limit of normal. Consider using levofloxacin as an alternative in these patients.

- Concurrent use with other drugs that prolong the QT interval. 


\section{Cautions}

- may impair performance of skilled tasks such as driving

- myasthenia gravis: risk of exacerbation

- G6PD deficiency: risk of haemolytic reactions when treated with quinolones

- sunlight: risk of photosensitivity reaction

- epilepsy/seizure activity: may induce convulsions in patients with or without history of convulsions, use with caution if epileptic or conditions predisposing seizures

- liver disease: cases of fulminant hepatitis potentially leading to liver failure (including fatal cases) have been reported

- serious bullous skin reactions: risk of Stevens-Johnson syndrome or toxic epidermal necrolysis

- peripheral neuropathy: sensorimotor polyneuropathy resulting in paraesthesias, hypoaesthesias, dysaesthesias or weakness.

\section{LABORATORY INFORMATION}

Please find up-to-date information at www.assayfinder.com regarding individual providers of drug level monitoring tests. Click on the provider to discover contact details. Turnaround time varies depending on the test and whether it is run locally or sent to an external lab. By contacting laboratories in advance, turnaround time can significantly be reduced.

Sample type: serum.

Volume required: $2 \mathrm{~mL}$ ( $\mathrm{min} 0.1 \mathrm{~mL}$ ).

Sample container: plain glass or plastic (non-SST).

Container type: any.

Availability: NS.

Turnaround time: telephoned same day if received 09:0015:00 Monday Friday if advanced warning given. Written confirmation report will be sent by first class post.

The sample must be heat-treated before dispatch if HIV-positive.

Please telephone at least 1 day in advance of the sample. 


\section{RIFABUTIN}

\section{Dosage}

Adult: $5 \mathrm{mg} / \mathrm{kg}$ once a day (oral). Usual dose is $300 \mathrm{mg}$, although doses of up to $450 \mathrm{mg}$ are sometimes used.

Children 12-17 years): $5 \mathrm{mg} / \mathrm{kg}$ once a day (oral).

Children (1 month - 11 years): $5 \mathrm{mg} / \mathrm{kg}$ once a day (limited data).

\section{Preparations}

- Oral: $150 \mathrm{mg}$ capsules.

\section{DRUG LEVEL MONITORING}

Indications for monitoring

- known or suspected malabsorption

- poor treatment response.

Target level: $0.3-0.9 \mathrm{mg} / \mathrm{L}$ (peak)

\section{Timing of sample}

- 3 hours postdose

- repeat at 7 hours if suspect delayed.

\section{Frequency of levels}

- Drug levels need not be routinely measured.

\author{
ADVERSE EFFECTS \\ Common \\ - reddish discolouration of urine, sweat, sputum, tears \\ - haematological: neutropaenia \\ - gastrointestinal: anorexia, nausea, vomiting, heartburn \\ - hepatic: transient increases in LFTs \\ - ophthalmic: uveitis \\ - dermatological: rash.
}

Serious

- haematological: anaemia, neutropaenia, thrombocytopaenia.

\section{ADVERSE EFFECTS: MONITORING}

Routine toxicity monitoring tests (FBC, U\&Es, LFTs) should be performed intermittently throughout antibiotic treatment for NTM-pulmonary disease (unless more specific advice is outlined above).

\section{Interactions}

- antiarrhythmics: accelerated metabolism of disopyramide

- anticoagulants: accelerated metabolism of coumarins (eg, warfarin)

- antidiabetics: accelerated metabolism of tolbutamide and sulfonylureas (reduced effect)

- antiepileptics: reduced plasma concentration of carbamazepine and phenytoin

- antifungals: increased serum concentration of rifabutin with fluconazole, posaconazole and voriconazole, and possibly itraconazole. Reduced serum concentrations of itraconazole, posaconazole and voriconazole. If benefit outweighs the risk, monitor antifungal serum concentrations (increase dose of voriconazole) and monitor for rifabutin adverse effects such as leucopenia and uveitis
- antipsychotics: possible reduced plasma concentration of aripiprazole

- antivirals: Please seek advice from an HIV physician before considering starting rifampicin in patients on antiretrovirals due to the frequency of drug interactions: increased serum concentration of rifabutin when given with amprenavir, fosamprenavir/ritonavir, lopinavir/ritonavir, ritonavir and tipranavir/ritonavir. Reduce dose of rifabutin; consider alternative protease inhibitor to ritonavir

- atovaquone: reduced plasma concentrations of both rifabutin and atovaquone

- contraceptives: accelerated metabolism of oestrogens and progestogens (reduced contraceptive effect)

- corticosteroids: possible accelerated metabolism of corticosteroids (reduced effect)

- hormone replacement therapy (HRT): rifampicin would be expected to reduce the efficacy of HRT

- ivacaftor: reduced plasma concentration of ivacaftor (avoid concomitant use)

- macrolides: increased risk of neutropaenia with azithromycin; increased plasma concentration of rifabutin when taken with clarithromycin and possibly erythromycin (reduce dose of rifabutin)

- Orkambi (lumacaftor/ivacaftor): reduced plasma concentration of ivacaftor and reduced plasma concentration of rifabutin (avoid concomitant use)

- P-aminosalicylic acid: reduced absorption of rifamycins; give 8-12 hours apart

- sirolimus: reduced in plasma concentration of sirolimus

- tacrolimus: reduced in plasma concentration of tacrolimus.

This information is not inclusive of all drug interactions. Please discuss with a pharmacist.

\section{CONTRAINDICATIONS AND CAUTIONS}

\section{Contraindications}

- hypersensitivity: to rifabutin or other rifamycins

- pregnancy

- breast feeding.

\section{Cautions}

- liver disease: use cautiously and monitor LFTs

- renal disease: reduce dose in severe renal impairment.

\section{LABORATORY INFORMATION}

Please find up-to-date information at www.assayfinder.com regarding individual providers of drug level monitoring tests. Click on the provider to discover contact details. Turnaround time varies depending on the test and whether it is run locally or sent to an external lab. By contacting laboratories in advance, turnaround time can significantly be reduced.

Sample type: serum.

Volume required: $2 \mathrm{~mL}$.

Sample container: plain (non-SST).

Container type: any.

Availability: office hours.

Turnaround time: 7 days. 


\section{RIFAMPICIN}

\section{Dosage}

Adults ( $<50 \mathrm{~kg}): 450 \mathrm{mg}$ once a day (oral or intravenous).

Adults $(50 \mathrm{~kg}+): 600 \mathrm{mg}$ once a day (oral or intravenous).

Children: $15 \mathrm{mg} / \mathrm{kg}$ ( $\max 450 \mathrm{mg}$ if $<50 \mathrm{~kg} ; 600 \mathrm{mg}$ if $50 \mathrm{~kg}+$ ) once a day (oral or intravenous) (Doses should be rounded up to facilitate administration of suitable volumes of liquid or an appropriate strength of capsule.)

Rifampicin should be taken 30-60 min before food, or 2 hours after food.

\section{Preparations}

- oral: $150 \mathrm{mg}, 300 \mathrm{mg}$ capsules

- $100 \mathrm{mg} / 5 \mathrm{~mL}$ syrup

- Rifinah 300/150 tablets (rifampicin 300 mg, isoniazid $150 \mathrm{mg}$ )

- Rifinah 150/100 tablets (rifampicin 150 mg, isoniazid $100 \mathrm{mg}$ )

- parenteral: $600 \mathrm{mg}$ powder for reconstitution.

\section{DRUG LEVEL MONITORING}

Indications for monitoring

- known or suspected malabsorption

- poor treatment response.

Target level: $8-24 \mathrm{mg} / \mathrm{L}$ (peak).

\section{Timing of sample}

- 2 hours postdose

- repeat at 6 hours if suspect delayed.

\section{Frequency of levels}

- Drug levels need not be routinely measured.

\section{ADVERSE EFFECTS}

Common

- reddish discolouration of urine, sweat, sputum, tears

- gastrointestinal: anorexia, nausea, vomiting, heartburn

- hepatic: transient increases in LFTs

- flu-like syndrome.

\section{Serious}

- haematological: agranulocytosis (rare), haemolytic anaemia (rare, usually intermittent therapy), thrombocytopaenia (rare, usually high-dose/intermittent therapy)

- hepatic: hepatotoxicity (rare)

- renal: nephrotoxicity (rare).

\section{ADVERSE EFFECTS: MONITORING}

Routine toxicity monitoring tests (FBC, U\&Es, LFTs) should be performed intermittently throughout antibiotic treatment for NTM-pulmonary disease (unless more specific advice is outlined above).

\section{Interactions}

- analgesics: accelerated metabolism of opiates, resulting in reduced effect (eg, alfentanyl, codeine, fentanyl, methadone, morphine and possibly oxycodone)

- antacids: reduced absorption of rifampicin

- antiarrhythmics: accelerated metabolism of disopyramide

- antibacterials: reduced plasma concentrations of chloramphenicol, clarithromycin, dapsone, doxycycline, linezolid and trimethoprim
- anticoagulants: reduced plasma concentration of apixaban, dabigatran and rivaroxaban; accelerated metabolism of coumarins (eg, warfarin)

- antidiabetics: accelerated metabolism of tolbutamide and sulfonylureas (reduced effect); reduced effect of linagliptin, nateglinide and repaglinide

- antiepileptics: reduced plasma concentration of lamotrigine and phenytoin; phenobarbital possibly reduces plasma concentration of rifampicin

- antifungals: accelerated metabolism of ketoconazole, fluconazole, itraconazole, posaconazole, terbinafine and voriconazole (reduced plasma concentrations; avoid concomitant use of rifampicin with itraconazole or voriconazole); rifampicin initially increases then decreases caspofungin levels (consider increasing caspofungin dose)

- antimalarials: reduced plasma concentration of mefloquine (avoid use) and quinine

- antipsychotics: accelerated metabolism of haloperidol and possibly aripiprazole and clozapine

- antivirals: reduced plasma concentration of atazanavir, darunavir, fosamprenavir, lopinavir, nelfinavir, nevirapine, rilpivirine, saquinavir and telaprevir (avoid concomitant use), and possibly abacavir, boceprevir, ritonavir and tipranavir; rifampicin also reduces plasma concentration of efavirenz (increase dose of efavirenz), maraviroc and raltegravir (consider increasing doses); accelerated metabolism of indinavir (avoid concomitant use)

- atovaquone: reduced plasma concentrations of atovaquone; increased plasma concentration of rifampicin (avoid concomitant use)

- bosentan: reduced plasma concentration of bosentan (avoid concomitant use)

- calcium channel blockers: accelerated metabolism of diltiazem, nifedipine, nimodipine and verapamil (significant reduction in plasma concentrations), and possibly isradipine and nicardipine

- ciclosporin: accelerated metabolism of ciclosporin (reduced plasma concentration)

- contraceptives: accelerated metabolism of oestrogens and progestogens (reduced contraceptive effect); avoid use of combined hormonal contraception (oral, patch or vaginal ring) and progestogen-only contraception (pill and implant); suitable alternatives include barrier methods, copper-bearing intrauterine system or progestogen-only injectable (depot medroxyprogesterone acetate, norethisterone enanthate or levonorgestrel-releasing intrauterine system, which can be continued at the usual dose and dosing/replacement interval of 12 weeks, 8 weeks and 5 years, respectively)

- corticosteroids: accelerated metabolism of corticosteroids (reduced effect)

- diuretics: reduced plasma concentration of eplerenone (avoid concomitant use)

- HRT: rifampicin would be expected to reduce the efficacy of HRT

- ivacaftor: reduced plasma concentration of ivacaftor (avoid concomitant use)

- mycophenolate: reduced plasma concentration of active metabolite of mycophenolate

- Orkambi (lumacaftor/ivacaftor): reduced plasma concentrations of ivacaftor (avoid concomitant use)

- P-aminosalicylic acid: reduced absorption of rifamycins; give 8-12 hours apart 


\section{BTS guideline}

- ranolazine: reduced plasma concentration of ranolazine (avoid concomitant use)

- sirolimus: reduced in plasma concentration of sirolimus

- tacrolimus: reduced in plasma concentration of tacrolimus

- tadalafil: reduced plasma concentration of tadalafil (avoid concomitant use)

- theophylline: accelerated metabolism of theophylline (reduced plasma concentration)

- ticagrelor: reduced plasma concentration of ticagrelor.

This information is not inclusive of all drug interactions.

Please discuss with a pharmacist.

\section{CONTRAINDICATIONS AND CAUTIONS}

\section{Contraindications}

- hypersensitivity: to rifampicin or other rifamycins

- liver disease: avoid if jaundiced

- drug interactions: avoid concomitant use with saquinavir or ritonavir.

\section{Cautions}

- liver disease: use cautiously and monitor LFTs; hyperbilirubinaemia may occur early in treatment in some patients due to competition between rifampicin and bilirubin for hepatic excretion.

\section{LABORATORY INFORMATION}

Please find up-to-date information at www.assayfinder.com regarding individual providers of drug level monitoring tests. Click on the provider to discover contact details. Turnaround time varies depending on the test and whether it is run locally or sent to an external lab. By contacting laboratories in advance, turnaround time can significantly be reduced.

Sample type: serum.

Volume required: Please note that rifampicin binds to glass and plastics and therefore there may be a significant loss of drug if a small volume of serum is dispatched in a relatively large container. Please try and fill the container to $2 / 3-3 / 4$ its capacity.

Sample container: plain glass or plastic (non-SST).

Container type: any.

Availability: office hours.

Turnaround time: telephoned same day if received 09:0015:00 Monday to Friday if advanced warning given. Written confirmation report will be sent by first class post.

The sample must be heat-treated before dispatch if HIV-positive.

Please telephone at least 1 day in advance of the sample. 


\section{STREPTOMYCIN}

Please note streptomycin is not licensed in the UK.

\section{Preparations}

- parenteral: $1 \mathrm{~g}$ powder for reconstitution for injection (unlicensed medicine).

\section{DOSAGE}

For intramuscular administration only. There is experience of using streptomycin as an intravenous infusion, but the prescriber should ensure the streptomycin preparation used is suitable for intravenous administration.

Streptomycin is usually given once daily for an initial period (usually 1 month), then the frequency may be reduced to three times weekly.

Adults: $15 \mathrm{mg} / \mathrm{kg}$ daily (usual maximum $1 \mathrm{~g}$ daily, but can be increased if necessary in large muscular adults). After initial period: $15 \mathrm{mg} / \mathrm{kg}$ three times per week.

Age $>59$ years: $10 \mathrm{mg} / \mathrm{kg}$ daily (maximum $750 \mathrm{mg}$ daily). After initial period: $15 \mathrm{mg} / \mathrm{kg}$ three times per week.

Renal failure: $12-15 \mathrm{mg} / \mathrm{kg}$ two to three times a week. Please discuss with a pharmacist.

Obesity: Use ideal body weight plus $40 \%$ of the excess weight in markedly obese patients. The adjusted dose is due to the decreased distribution of extracellular fluids in adipose tissues.

- male ideal body weight $(\mathrm{kg})=50+(2.3 \times$ height in $\mathrm{cm}$ above 152.4)/2.54

- female ideal body weight $(\mathrm{kg})=45.5+(2.3 \times$ height in $\mathrm{cm}$ above 152.4)/2.54

Adjust dose and/or frequency according to serum streptomycin concentration (see below).

Children: $15 \mathrm{mg} / \mathrm{kg}$ daily (usual maximum $1 \mathrm{~g}$ daily). After initial period: $15 \mathrm{mg} / \mathrm{kg}$ three times per week.

Adjust dose and/or frequency according to serum streptomycin concentration (see below).

\section{DRUG LEVEL MONITORING}

Indications for monitoring

- ensure therapeutic dose

- ensure renal clearance, especially in at-risk patients (eg, renal impairment, elderly).

Target level: $<5 \mathrm{mg} / \mathrm{L}$ (trough), 25-35 mg/L (peak).

\section{Timing of sample}

- predose

- 60 min after infusion ends.

\section{Frequency of levels}

- peak serum level in first week, repeat if poor response

- trough serum levels weekly for 4 weeks, fortnightly for 4 weeks, then monthly if stable.

\section{ADVERSE EFFECTS}

\section{Common}

- nephrotoxicity: accumulation if renal impairment

- ototoxicity: irreversible vestibulocochlear nerve damage

- hypersensitivity skin reactions: rashes, urticaria, erythroderma

- drug-induced eosinophilia (usually subsides with intermittent dosing).
Serious

- endocrine: hypocalcaemia, hypomagnesaemia and hypokalaemia

- immunological: anaphylaxis (uncommon)

- neurological: neuromuscular blockade and respiratory paralysis (more common in neuromuscular disease; usually dose-related and self-limiting)

- audiological: ototoxicity-auditory > vestibular (higher with prolonged use and older age)

- renal: nephrotoxicity (higher with prolonged use).

\section{ADVERSE EFFECTS: MONITORING}

Renal, auditory and vestibular monitoring is essential.

Renal function

- month $1=$ twice weekly

- month 2 =weekly

- month3 to end of treatment with anaminoglycoside $=2$ weekly. Consider increasing frequency of monitoring if evidence of renal impairment.

Loss of hearing usually occurs first and is detected by regular audiometric testing. Vertigo, loss of balance and auditory disturbances are also signs of ototoxicity. Ototoxicity on audiogram is defined as a $20 \mathrm{~dB}$ loss from baseline at any one test frequency or a $10 \mathrm{~dB}$ loss at any two adjacent test frequencies.

We recommend that patients have baseline audiometry and then monthly reviews until treatment with aminoglycoside ceases. A final audiometry review should be offered 2 months after the final dose.

Routine toxicity monitoring tests (FBC, U\&Es, LFTs) should be performed intermittently throughout antibiotic treatment for NTM-pulmonary disease (unless more specific advice is outlined above).

\section{Interactions}

- increased risk of nephrotoxicity if given with capreomycin, cephalosporins, ciclosporin, colistimethate sodium and tacrolimus

- increased risk of ototoxicity if given with loop diuretics

- increased risk of hypocalcaemia with bisphosphonates.

This information is not inclusive of all drug interactions. Please discuss with a pharmacist.

\section{CONTRAINDICATIONS AND CAUTIONS}

Contraindications

- hypersensitivity: to streptomycin or other aminoglycosides

- myasthenia gravis: as aminoglycosides may impair neuromuscular transmission

- pregnancy: risk of vestibular or auditory nerve damage to infant if used in second or third trimester.

\section{Cautions}

- obese: use ideal weight for height to calculate dose and monitor serum streptomycin levels closely

- elderly: nephrotoxicity and ototoxicity common in the elderly; monitor and reduce dose if necessary

- renal disease: use with caution; reduce the frequency of dosing and monitor serum concentrations.

\section{LABORATORY INFORMATION}

Please find up-to-date information at www.assayfinder.com regarding individual providers of drug level monitoring tests. Click on the provider to discover contact details. Turnaround 


\section{BTS guideline}

time varies depending on the test and whether it is run locally or sent to an external lab. By contacting laboratories in advance, turnaround time can significantly be reduced.

Sample type: serum.

Volume required: $2 \mathrm{~mL}$ (min $0.1 \mathrm{~mL}$ ).

Sample container: plain glass or plastic (non-SST).

Container type: any.

Availability: office hours.
Turnaround time: telephoned same day if received 09:0015:00, Monday to Friday if advanced warning given. Written confirmation report will be sent by first class post.

The sample must be heat-treated before dispatch if HIV-positive. 


\section{TIGECYCLINE}

Please note tigecycline is not licensed for the treatment of NTM in the UK.

\section{Dosage}

Adults: $100 \mathrm{mg}$ loading dose, then $50 \mathrm{mg}$ twice daily.

Some studies have used lower doses, for example, $50 \mathrm{mg} / \mathrm{day}$ to improve tolerance, although once-daily dosing is unproven clinically.

Children 12-18 years: $100 \mathrm{mg}$ loading dose, then $50 \mathrm{mg}$ twice daily (intravenous).

Children $8-11$ years: $1.2 \mathrm{mg} / \mathrm{kg}$ twice daily (maximum $50 \mathrm{mg}$ twice daily) (intravenous).

Children $<8$ years: lack of data.

Paediatric doses: (Note: Limited data on evidence for dosing in NTM. These doses are based on tigecycline dosing for respiratory tract infections in the latest BNF for children, December 2015 update.)

\section{Preparations}

- $50 \mathrm{mg}$ powder for solution for infusion.

\section{DRUG LEVEL MONITORING}

- Drug levels cannot currently be performed in the UK.

\section{ADVERSE EFFECTS}

\section{Common}

- dermatological: pruritus, rash

- gastrointestinal: nausea, vomiting, diarrhoea, abdominal pain, dyspepsia, anorexia

- haematological: prolonged activated partial thromboplastin time (aPTT), prolonged prothrombin time (PT)

- hepatic: elevated LFTs

- infections: sepsis/septic shock, pneumonia, abscess, infections

- investigations: elevated amylase, elevated blood urea nitrogen

- metabolic: hypoglycaemia, hypoproteinaemia

- neurological: dizziness, headache

- vascular: phlebitis.

\section{Serious}

- dermatological: severe skin reactions, including StevensJohnson syndrome, photosensitivity

- haematological: thrombocytopaenia

- hepatic: hepatic failure, acute pancreatitis

- immunological: anaphylaxis

- infections: sepsis/septic shock, pneumonia, abscess, infections, Pseudomembranous colitis neurological: benign intracranial hypertension.

\section{ADVERSE EFFECTS: MONITORING}

LFTs, FBC, clotting and amylase should be monitored at baseline and regularly while on treatment.

Routine toxicity monitoring tests (FBC, U\&Es, LFTs) should be performed intermittently throughout antibiotic treatment for NTM-pulmonary disease (unless more specific advice is outlined above).

\section{Interactions}

- anticoagulants: prolonged prothrombin time in patients taking warfarin (monitor INR and reduce warfarin dose)

- ciclosporin: (limited data): increased ciclosporin serum concentrations may occur (monitor ciclosporin levels closely and adjust dose)

- tacrolimus: (limited data): increased tacrolimus serum concentrations may occur (monitor tacrolimus levels closely and adjust dose).

This information is not inclusive of all drug interactions. Please discuss with a pharmacist.

\section{CONTRAINDICATIONS AND CAUTIONS}

\section{Contraindications}

- hypersensitivity: to tigecycline or to tetracyclines.

\section{Cautions}

- in clinical studies for complicated skin and soft tissue infections, complicated intra-abdominal infections, diabetic foot infections, nosocomial pneumonia and studies in resistant pathogens, a numerically higher mortality rate was observed in patients treated with tigecycline compared with comparator antibiotics; the cause for this is unclear, but poorer efficacy and safety cannot be ruled out

- haematological: tigecycline may prolong PT and aPTT

- pregnancy and breast feeding (evidence of fetal harm in animal studies)

- children under 8 years due to risk of teeth discolouration.

\section{LABORATORY INFORMATION}

Please find up-to-date information at www.assayfinder.com regarding individual providers of drug level monitoring tests. Click on the provider for contact details. Turnaround time varies depending on the test and whether it is run locally or sent to an external lab. By contacting laboratories in advance, turnaround time can significantly be reduced. 


\section{SECTION 19: RESEARCH RECOMMENDATIONS NTM epidemiology}

1. What are the risk factors for developing NTM-pulmonary disease?

Why is this important? We need to understand who is at high risk of NTM infection in order (1) to alert clinicians to consider NTM-pulmonary disease in these groups and diagnose it promptly and (2) to evaluate the benefit of screening for NTM in high-risk groups.

2. What are the mechanisms contributing to patient-to-patient transmission of NTM?

Why is this important? Given the growing evidence supporting M. abscessus transmission between individuals with CF, it is essential that we evaluate whether person-to-person transmission can occur in different patient groups and with other NTM species, and define precisely the routes through which cross-infection can occur in order to minimise risk to individuals.

\section{NTM microbiology}

1. Can we develop more rapid identification of NTM species from respiratory samples?

Why is this important? There are currently significant delays in identification and speciation of mycobacteria from respiratory samples, which delay the diagnosis, and consequently the treatment, of NTM-pulmonary disease. The development and rigorous validation of non-culture-based detection and speciation methods would greatly impact on the management of NTM infection.

2. Can we better understand the role of DST in predicting treatment outcomes?

Why is this important? We currently do not understand the significance of in vitro susceptibility testing for most antibiotics in most NTM species in predicting clinical response to antibiotics. This potentially means we are prescribing ineffectual or inappropriate antibiotics and failing to give optimal drug regimens.

\section{NTM treatment}

1. Can we better understand the pathogenicity of specific NTM species and subspecies to facilitate treatment decision-making?

Why is this important? It is recognised, although incompletely understood, that the pathogenic potential of different NTM species varies considerably. A comprehensive analysis of the likelihood that an isolated NTM species or subspecies might cause progressive inflammatory lung disease would allow a more nuanced approach to the diagnosis of NTM-pulmonary disease and the decision about when and how to treat patients.

2. Are there more effective and more tolerable NTM treatment regimens?

Why is this important? We currently use combination antibiotic regimens that are often associated with significant toxicity, require prolonged administration, and particularly in the case of $M$. abscessus frequently fail. There remain considerable challenges in coordinating and funding multicentre studies of novel drugs or new combinations of existing drugs for NTM infections.

\footnotetext{
Author affiliations

${ }^{1}$ Cambridge Centre for Lung Infection, Papworth Hospital, Cambridge, UK

${ }^{2}$ Abertawe Bro Morgannwg University Health Board, Princess of Wales Hospital, Bridgend, UK

${ }^{3}$ Pharmacy Department, St James's University Hospital, Leeds, UK

${ }^{4}$ Institute of Cellular Medicine, Faculty of Medical Sciences, Newcastle University, Newcastle Upon Tyne, UK

${ }^{5}$ Manchester Royal Infirmary, Manchester, UK
}

${ }^{6}$ Scottish Mycobacteria Reference Laboratory, Royal Infirmary of Edinburgh, Edinburgh, UK

${ }^{7}$ Department of Respiratory Medicine, Western General Hospital. Edinburgh, UK

${ }^{8}$ Host Defence Unit, Royal Brompton Hospital, and National, Heart and Lung Institute, Imperial College, London, UK

${ }^{9}$ Department of Respiratory Medicine, Guys and St Thomas NHS Foundation Trust, London, UK

${ }^{10}$ British Thoracic Society, London UK

${ }^{11}$ Division of Infection, Immunity \& Respiratory Medicine, University of Manchester,

Wythenshawe Hospital, Manchester, UK

${ }^{12}$ Great Ormond Street Hospital for Children NHS Foundation Trust, London, UK

${ }^{13}$ North Bristol Lung Centre, Southmead Hospital, Bristol, UK

${ }^{14}$ Queen Alexandra Hospital, Portsmouth, UK

${ }^{15}$ Department of Medicine, University of Cambridge, UK

Acknowledgements The British Thoracic Society and the Guideline Group members are particularly grateful to Dr lan Campbell and Dr Michael Ruddy, who contributed to the early work of the guideline group; Dr Ewan Olson; and members of the BTS Standards of Care Committee.

Competing interests JB has received honorarium funding from AZ. TC has received funding from Chiesi. AF has received funding from GSK. ARF has received funding from Insmed. CSH has received funding from Aradigm, Chiesi, Gilead, Insmed, Raptor, Teva, Vertex, Zambon. ML has received funding from Insmed, Pulmocide, Bayer, Chiesi and Raptor. RW has received funding from Bayer.

Provenance and peer review Not commissioned; externally peer reviewed.

(c) Article author(s) (or their employer(s) unless otherwise stated in the text of the article) 2017. All rights reserved. No commercial use is permitted unless otherwise expressly granted.

\section{REFERENCES}

1 Griffith DE, Aksamit T, Brown-Elliott BA, et al. An official ATS/IDSA statement: diagnosis, treatment, and prevention of nontuberculous mycobacterial diseases. Am J Respir Crit Care Med 2007:175:367-416.

2 NICE. Tuberculosis: clinical diagnosis and management of tuberculosis, and measures for its prevention and control. Secondary Tuberculosis: clinical diagnosis and management of tuberculosis, and measures for its prevention and control. 2011 https://www.nice.org.uk/guidance/cg117

3 Management of opportunist mycobacterial infections: Joint Tuberculosis Committee Guidelines 1999. Subcommittee of the Joint Tuberculosis Committee of the British Thoracic Society. Thorax 2000;55:210-8.

4 Matveychuk A, Fuks L, Priess R, et al. Clinical and radiological features of Mycobacterium kansasii and other NTM infections. Respir Med 2012;106:1472-7.

5 Prince DS, Peterson DD, Steiner RM, et al. Infection with Mycobacterium avium complex in patients without predisposing conditions. $N$ Engl J Med 1989;321:863-8

6 Prevots DR, Shaw PA, Strickland D, et al. Nontuberculous mycobacterial lung disease prevalence at four integrated health care delivery systems. Am J Respir Crit Care Med 2010;182:970-6.

7 Hoefsloot W, van Ingen J, Andrejak C, et al. The geographic diversity of nontuberculous mycobacteria isolated from pulmonary samples: an NTM-NET collaborative study. Eur Respir J 2013;42:1604-13.

8 Bodle EE, Cunningham JA, Della-Latta P, et al. Epidemiology of nontuberculous mycobacteria in patients without HIV infection, New York City. Emerg Infect Dis 2008;14:390-6.

9 Adjemian J, Olivier KN, Seitz AE, et al. Prevalence of nontuberculous mycobacteria lung disease in U.S. Medicare beneficiaries. Am J Respir Crit Care Med 2012:185:881-6.

10 Adjemian J, Olivier KN, Seitz AE, et al. Spatial clusters of nontuberculous mycobacterial lung disease in the United States. Am J Respir Crit Care Med 2012;186:553-8

11 Pedro HS, Pereira MI, Goloni MR, et al. Nontuberculous mycobacteria isolated in São José do Rio Preto, Brazil between 1996 and 2005. J Bras Pneumo/ 2008;34:950-5.

12 Davies BS, Roberts CH, Kaul S, et al. Non-tuberculous slow-growing mycobacterial pulmonary infections in non-HIV-infected patients in south London. Scand J Infect Dis 2012;44:815-9.

13 Moore JE, Kruijshaar ME, Ormerod LP, et al. Increasing reports of non-tuberculous mycobacteria in England, Wales and Northern Ireland, 1995-2006. BMC Public Health 2010;10:612.

14 Gerogianni I, Papala M, Kostikas K, et al. Epidemiology and clinical significance of mycobacterial respiratory infections in Central Greece. Int J Tuberc Lung Dis 2008;12:807-12

15 van Ingen J, Bendien SA, de Lange WC, et al. Clinical relevance of non-tuberculous mycobacteria isolated in the Nijmegen-Arnhem region, The Netherlands. Thorax 2009;64:502-6 
16 Aliyu G, El-Kamary SS, Abimiku A, et al. Prevalence of non-tuberculous mycobacterial infections among tuberculosis suspects in Nigeria. PLoS One 2013;8:e63170.

17 Asiimwe BB, Bagyenzi GB, Ssengooba W, et al. Species and genotypic diversity of non-tuberculous mycobacteria isolated from children investigated for pulmonary tuberculosis in rural Uganda. BMC Infect Dis 2013;13:88.

18 Simons S, van Ingen J, Hsueh PR, et al. Nontuberculous mycobacteria in respiratory tract infections, eastern Asia. Emerg Infect Dis 2011;17:343-9.

19 Morimoto K, Iwai K, Uchimura K, et al. A steady increase in nontuberculous mycobacteriosis mortality and estimated prevalence in Japan. Ann Am Thorac Soc 2014;11:1-8.

20 Lai CC, Tan CK, Chou CH, et al. Increasing incidence of nontuberculous mycobacteria, Taiwan, 2000-2008. Emerg Infect Dis 2010;16:294-6.

21 Jing $\mathrm{H}$, Wang $\mathrm{H}$, Wang $\mathrm{Y}$, et al. Prevalence of nontuberculous mycobacteria infection, China, 2004-2009. Emerg Infect Dis 2012;18:527-8.

22 Shao $Y$, Chen $C$, Song $H$, et al. The epidemiology and geographic distribution of nontuberculous mycobacteria clinical isolates from sputum samples in the eastern region of China. PLoS Negl Trop Dis 2015;9:e0003623.

23 Prevots DR, Marras TK. Epidemiology of human pulmonary infection with nontuberculous mycobacteria: a review. Clin Chest Med 2015;36:13-34.

24 Falkinham JO. Environmental sources of nontuberculous mycobacteria. Clin Chest Med 2015;36:35-41.

25 Thomson RM, NwgaQTC C. Queensland Mycobacterial Reference L. Changing epidemiology of pulmonary nontuberculous mycobacteria infections. Emerg Infect Diseases 2010;16:1576-83.

26 Chou MP, Clements AC, Thomson RM. A spatial epidemiological analysis of nontuberculous mycobacterial infections in Queensland, Australia. BMC Infect Dis $2014 ; 14: 279$.

27 Koh WJ, Chang B, Jeong BH, et al. Increasing Recovery of Nontuberculous Mycobacteria from Respiratory Specimens over a 10-Year Period in a Tertiary Referral Hospital in South Korea. Tuberc Respir Dis 2013;75:199-204.

28 Andréjak C, Thomsen VØ, Johansen IS, et al. Nontuberculous pulmonary mycobacteriosis in Denmark: incidence and prognostic factors. Am J Respir Crit Care Med 2010;181:514-21.

29 Marras TK, Mendelson D, Marchand-Austin A, et al. Pulmonary nontuberculous mycobacterial disease, Ontario, Canada, 1998-2010. Emerg Infect Dis 2013;19:1889-91.

30 Shah NM, Davidson JA, Anderson LF, et al. Pulmonary Mycobacterium aviumintracellulare is the main driver of the rise in non-tuberculous mycobacteria incidence in England, Wales and Northern Ireland, 2007-2012. BMC Infect Dis 2016;16:195.

31 Renna M, Schaffner C, Brown K, et al. Azithromycin blocks autophagy and may predispose cystic fibrosis patients to mycobacterial infection. J Clin Invest 2011:121:3554-63.

32 Khan K, Wang J, Marras TK. Nontuberculous mycobacterial sensitization in the United States: national trends over three decades. Am J Respir Crit Care Med 2007;176:306-13.

33 Brode SK, Daley CL, Marras TK. The epidemiologic relationship between tuberculosis and non-tuberculous mycobacterial disease: a systematic review. Int J Tuberc Lung Dis 2014;18:1370-7.

34 Falkinham JO. Nontuberculous mycobacteria from household plumbing of patients with nontuberculous mycobacteria disease. Emerg Infect Dis 2011;17:419-24.

35 Falkinham JO. Current Epidemiologic Trends of the Nontuberculous Mycobacteria (NTM). Curr Environ Health Rep 2016:3:161-7.

36 Feazel LM, Baumgartner LK, Peterson KL, et al. Opportunistic pathogens enriched in showerhead biofilms. Proc Natl Acad Sci U S A 2009;106:16393-9.

37 Thomson R, Tolson C, Carter R, et al. Isolation of nontuberculous mycobacteria (NTM) from household water and shower aerosols in patients with pulmonary disease caused by NTM. J Clin Microbiol 2013;51:3006-11.

38 Catherinot $\mathrm{E}$, Roux AL, Vibet MA, et al. Mycobacterium avium and Mycobacterium abscessus complex target distinct cystic fibrosis patient subpopulations. J Cyst Fibros 2013;12:74-80

39 Bryant JM, Grogono DM, Parkhill J, et al. Transmission of $\mathrm{M}$ abscessus in patients with cystic fibrosis - Authors' reply. Lancet 2013;382:504.

40 Bryant JM, Grogono DM, Greaves D, et al. Whole-genome sequencing to identify transmission of Mycobacterium abscessus between patients with cystic fibrosis: a retrospective cohort study. Lancet 2013;381:1551-60.

41 Bryant JM, Grogono DM, Rodriguez-Rincon D, et al. Emergence and spread of a human-transmissible multidrug-resistant nontuberculous mycobacterium. Science 2016;354:751-7.

42 Donohue MJ, Mistry JH, Donohue JM, et al. Increased Frequency of Nontuberculous Mycobacteria Detection at Potable Water Taps within the United States. Environ Sci Technol 2015;49:6127-33.

43 Levy I, Grisaru-Soen G, Lerner-Geva L, et al. Multicenter cross-sectional study of nontuberculous mycobacterial infections among cystic fibrosis patients, Israel. Emerg Infect Dis 2008;14:378-84.

44 Bloch KC, Zwerling L, Pletcher MJ, et al. Incidence and clinical implications of isolation of Mycobacterium kansasii: results of a 5-year, population-based study. Ann Intern Med 1998;129:698-704.
45 Hojo M, likura M, Hirano S, et al. Increased risk of nontuberculous mycobacterial infection in asthmatic patients using long-term inhaled corticosteroid therapy. Respirology 2012;17:185-90.

46 Henry MT, Inamdar L, O'Riordain D, et al. Nontuberculous mycobacteria in non-HIV patients: epidemiology, treatment and response. Eur Respir J 2004;23:741-6.

47 Marras TK, Mehta M, Chedore P, et al. Nontuberculous mycobacterial lung infections in Ontario, Canada: clinical and microbiological characteristics. Lung 2010;188:289-99.

48 Chan ED, Kaminska AM, Gill W, et al. Alpha-1-antitrypsin (AAT) anomalies are associated with lung disease due to rapidly growing mycobacteria and AAT inhibits Mycobacterium abscessus infection of macrophages. Scand I Infect Dis 2007;39:690-6.

49 Andréjak C, Nielsen R, Thomsen VØ, et al. Chronic respiratory disease, inhaled corticosteroids and risk of non-tuberculous mycobacteriosis. Thorax 2013:68:256-62

50 McGrath EE, Bardsley P. An association between Mycobacterium malmoense and coal workers' pneumoconiosis. Lung 2009;187:51-4.

51 Adjemian J, Olivier KN, Prevots DR. Nontuberculous mycobacteria among patients with cystic fibrosis in the United States: screening practices and environmental risk. Am J Respir Crit Care Med 2014;190:581-6.

52 Leung JM, Olivier KN. Nontuberculous mycobacteria: the changing epidemiology and treatment challenges in cystic fibrosis. Curr Opin Pulm Med 2013;19:662-9.

53 Griffith DE, Aksamit TR, Timothy R. Bronchiectasis and nontuberculous mycobacterial disease. Clin Chest Med 2012;33:283-95.

54 Mirsaeidi M, Hadid W, Ericsoussi B, et al. Non-tuberculous mycobacterial disease is common in patients with non-cystic fibrosis bronchiectasis. Int J Infect Dis 2013;17:e1000-4

55 Wickremasinghe $\mathrm{M}$, Ozerovitch $\mathrm{LJ}$, Davies $\mathrm{G}$, et al. Non-tuberculous mycobacteria in patients with bronchiectasis. Thorax 2005;60:1045-51

56 Noone PG, Leigh MW, Sannuti A, et al. Primary ciliary dyskinesia: diagnostic and phenotypic features. Am J Respir Crit Care Med 2004;169:459-67.

57 Mussaffi H, Rivlin J, Shalit I, et al. Nontuberculous mycobacteria in cystic fibrosis associated with allergic bronchopulmonary aspergillosis and steroid therapy. Eur Respir J 2005:25:324-8.

58 Kunst $H$, Wickremasinghe $M$, Wells $A$, et al. Nontuberculous mycobacterial disease and Aspergillus-related lung disease in bronchiectasis. Eur Respir J 2006:28:352-7.

59 Fowler CJ, Olivier KN, Leung JM, et al. Abnormal nasal nitric oxide production, ciliary beat frequency, and Toll-like receptor response in pulmonary nontuberculous mycobacterial disease epithelium. Am J Respir Crit Care Med 2013;187:1374-81.

60 Jang MA, Kim SY, Jeong BH, et al. Association of CFTR gene variants with nontuberculous mycobacterial lung disease in a Korean population with a low prevalence of cystic fibrosis. J Hum Genet 2013;58:298-303.

61 Chan ED, Iseman MD. Underlying host risk factors for nontuberculous mycobacterial lung disease. Semin Respir Crit Care Med 2013;34:110-23.

62 Reich JM, Johnson RE. Mycobacterium avium complex pulmonary disease presenting as an isolated lingular or middle lobe pattern. The Lady Windermere syndrome. Chest 1992;101:1605-9.

63 Colombo RE, Hill SC, Claypool RJ, et al. Familial clustering of pulmonary nontuberculous mycobacterial disease. Chest 2010;137:629-34.

64 Kim RD, Greenberg DE, Ehrmantraut ME, et al. Pulmonary nontuberculous mycobacterial disease: prospective study of a distinct preexisting syndrome. Am J Respir Crit Care Med 2008;178:1066-74

65 Leung JM, Fowler C, Smith C, et al. A familial syndrome of pulmonary nontuberculous mycobacteria infections. Am J Respir Crit Care Med 2013;188:1373-6.

66 Kartalija M, Ovrutsky AR, Bryan CL, et al. Patients with nontuberculous mycobacterial lung disease exhibit unique body and immune phenotypes. Am J Respir Crit Care Med 2013;187:197-205

67 Szymanski EP, Leung JM, Fowler CJ, et al. Pulmonary Nontuberculous Mycobacterial Infection. A Multisystem, Multigenic Disease. Am J Respir Crit Care Med 2015; 192:618-28.

68 Thomson RM, Armstrong JG, Looke DF, et al. acid suppression, and Mycobacterium avium complex pulmonary disease. Chest 2007;131:1166-72.

69 Koh WJ, Lee JH, Kwon YS, et al. Prevalence of gastroesophageal reflux disease in patients with nontuberculous mycobacterial lung disease. Chest 2007;131:1825-30.

70 Liao TL, Lin CF, Chen YM, et al. Risk Factors and Outcomes of Nontuberculous Mycobacterial Disease among Rheumatoid Arthritis Patients: A Case-Control study in a TB Endemic Area. Sci Rep 2016;6:29443.

71 Liao TL, Lin CH, Shen GH, et al. Risk for Mycobacterial Disease among Patients with Rheumatoid Arthritis, Taiwan, 2001-2011. Emerg Infect Dis 2015;21:1387-95.

72 Brode SK, Jamieson FB, Ng R, et al. Risk of mycobacterial infections associated with rheumatoid arthritis in Ontario, Canada. Chest 2014;146:563-72.

73 Gelder CM, Hart KW, Williams OM, et al. Vitamin D receptor gene polymorphisms and susceptibility to Mycobacterium malmoense pulmonary disease. $J$ Infect Dis 2000;181:2099-102.

74 Jeon K, Kim SY, Jeong BH, et al. Severe vitamin D deficiency is associated with non-tuberculous mycobacterial lung disease: a case-control study. Respirology 2013; 18:983-8 
75 Okumura M, Iwai K, Ogata $\mathrm{H}$, et al. Clinical factors on cavitary and nodular bronchiectatic types in pulmonary Mycobacterium avium complex disease. Intern Med 2008;47:1465-72.

76 Roux AL, Catherinot E, Soismier N, et al. Comparing Mycobacterium massiliense and Mycobacterium abscessus lung infections in cystic fibrosis patients. J Cyst Fibros 2015;14:63-9.

77 Cegielski JP, McMurray DN. The relationship between malnutrition and tuberculosis: evidence from studies in humans and experimental animals. Int I Tuberc Lung Dis 2004;8:286-98.

78 Lee SJ, Ryu YJ, Lee JH, et al. The impact of low subcutaneous fat in patients with nontuberculous mycobacterial lung disease. Lung 2014;192:395-401.

79 Wu UI, Holland SM. Host susceptibility to non-tuberculous mycobacterial infections. Lancet Infect Dis 2015;15:968-80.

80 Lake MA, Ambrose LR, Lipman MC, et al. "Why me, why now?" Using clinical immunology and epidemiology to explain who gets nontuberculous mycobacterial infection. BMC Med 2016;14:54.

81 Sologuren I, Boisson-Dupuis S, Pestano J, et al. Partial recessive IFN- $\gamma$ R1 deficiency: genetic, immunological and clinical features of 14 patients from 11 kindreds. Hum Mol Genet 2011:20:1509-23.

82 Kristensen IA, Veirum JE, Møller BK, et al. Novel STAT1 alleles in a patient with impaired resistance to mycobacteria. J Clin Immunol 2011;31:265-71.

83 Hsu AP, Sampaio EP, Khan J, et al. Mutations in GATA2 are associated with the autosomal dominant and sporadic monocytopenia and mycobacterial infection (MonoMAC) syndrome. Blood 2011;118:2653-5.

84 Sapkota BR, Hijikata M, Matsushita I, et al. Association of SLC11A1 (NRAMP1) polymorphisms with pulmonary Mycobacterium avium complex infection. Hum Immunol 2012;73:529-36.

85 Henkle E, Winthrop KL. Nontuberculous mycobacteria infections in immunosuppressed hosts. Clin Chest Med 2015;36:91-9.

86 Browne SK. Anticytokine autoantibody-associated immunodeficiency. Annu Rev Immunol 2014;32:635-57.

87 Dirac MA, Horan KL, Doody DR, et al. Environment or host?: A case-control study of risk factors for Mycobacterium avium complex lung disease. Am J Respir Crit Care Med 2012;186:684-91

88 Winthrop KL, Chang E, Yamashita S, et al. Nontuberculous mycobacteria infections and anti-tumor necrosis factor-alpha therapy. Emerg Infect Dis 2009;15:1556-61.

89 Viviani L, Harrison MJ, Zolin A, et al. Epidemiology of nontuberculous mycobacteria (NTM) amongst individuals with cystic fibrosis (CF). J Cyst Fibros 2016;15:619-23.

90 Girón RM, Máiz L, Barrio I, et al. [Nontuberculous mycobacterial infection in patients with cystic fibrosis: a multicenter prevalence study]. Arch Bronconeumol 2008;44:679-84.

91 Radhakrishnan DK, Yau Y, Corey M, et al. Non-tuberculous mycobacteria in children with cystic fibrosis: isolation, prevalence, and predictors. Pediatr Pulmonol 2009:44:1100-6.

92 Verregghen M, Heijerman HG, Reijers M, et al. Risk factors for Mycobacterium abscessus infection in cystic fibrosis patients; a case-control study. J Cyst Fibros 2012;11:340-3.

93 Coolen N, Morand P, Martin C, et al. Reduced risk of nontuberculous mycobacteria in cystic fibrosis adults receiving long-term azithromycin. J Cyst Fibros 2015;14:594-9.

94 Catherinot $\mathrm{E}$, Roux AL, Vibet MA, et al. Inhaled therapies, azithromycin and Mycobacterium abscessus in cystic fibrosis patients. Eur Respir J 2013;41:1101-6.

95 Binder AM, Adjemian J, Olivier KN, et al. Epidemiology of nontuberculous mycobacterial infections and associated chronic macrolide use among persons with cystic fibrosis. Am J Respir Crit Care Med 2013;188:807-12.

96 Falkinham JO. Epidemiology of infection by nontuberculous mycobacteria. Clin Microbiol Rev 1996;9:177-215.

97 Klausen J, Giese SB, Fuursted K, et al. Distribution of serotypes, IS901 and a 40 kDa protein in Mycobacterium avium complex strains isolated from man and animals in Denmark APMIS, et immunologica Scandinavica. 1997;105:277-82.

98 Feizabadi MM, Robertson ID, Cousins DV, et al. Genetic characterization of Mycobacterium avium isolates recovered from humans and animals in Australia. Epidemiol Infect 1996;116:41-9.

99 Olivier KN, Weber DJ, Wallace RJ, et al. Nontuberculous mycobacteria. I: multicenter prevalence study in cystic fibrosis. Am J Respir Crit Care Med 2003;167:828-34.

100 Sermet-Gaudelus I, Le Bourgeois M, Pierre-Audigier C, et al. Mycobacterium abscessus and children with cystic fibrosis. Emerg Infect Dis 2003:9:1587-91.

101 Aitken ML, Limaye A, Pottinger P, et al. Respiratory outbreak of Mycobacterium abscessus subspecies massiliense in a lung transplant and cystic fibrosis center. Am J Respir Crit Care Med 2012;185:231-2.

102 Davidson RM, Hasan NA, Reynolds PR, et al. Genome sequencing of Mycobacterium abscessus isolates from patients in the united states and comparisons to globally diverse clinical strains. J Clin Microbiol 2014;52:3573-82.

103 Harris KA, Underwood A, Kenna DT, et al. Whole-genome sequencing and epidemiological analysis do not provide evidence for cross-transmission of mycobacterium abscessus in a cohort of pediatric cystic fibrosis patients. Clin Infect Dis 2015;60:1007-16.

104 Foundation CF. Infection Prevention and Control Care Guidelines. Secondary Infection Prevention and Control Care Guidelines. 2014. https://www.cff.org/
For-Caregivers/Clinical-Care-Guidelines/Infection-Prevention-and-Control-CareGuidelines/

105 Group TUCFTMalCW. Mycobacterium abscessus Suggestions for infection prevention and control (Interim guidance - October 2013): Cystic Fibrosis Trust. 2013.

106 Tsukamura M. Diagnosis of disease caused by Mycobacterium avium complex. Chest 1991:99:667-9.

107 Lee MR, Yang CY, Shu CC, et al. Factors associated with subsequent nontuberculous mycobacterial lung disease in patients with a single sputum isolate on initial examination. Clin Microbiol Infect 2015;21:250.e1-250.e7.

108 Koh WJ, Chang B, Ko Y, et al. Clinical significance of a single isolation of pathogenic nontuberculous mycobacteria from sputum specimens. Diagn Microbiol Infect Dis 2013;75:225-6.

109 Martiniano SL, Sontag MK, Daley CL, et al. Clinical significance of a first positive nontuberculous mycobacteria culture in cystic fibrosis. Ann Am Thorac Soc 2014;11:36-44.

110 Tukey MH, Wiener RS. Population-based estimates of transbronchial lung biopsy utilization and complications. Respir Med 2012;106:1559-65.

111 Tanaka E, Amitani R, Niimi A, et al. Yield of computed tomography and bronchoscopy for the diagnosis of Mycobacterium avium complex pulmonary disease. Am J Respir Crit Care Med 1997;155:2041-6.

112 Sugihara E, Hirota N, Niizeki T, et al. Usefulness of bronchial lavage for the diagnosis of pulmonary disease caused by Mycobacterium avium-intracellulare complex (MAC) infection. J Infect Chemother 2003;9:328-32.

113 van Ingen J. Diagnosis of nontuberculous mycobacterial infections. Semin Respir Crit Care Med 2013;34:103-9.

114 Delogu G, Hermann JL. Mycobacterium species. ESCMID-SFM Manual of Microbiology 2010.

115 Traore I, Slosárek M. Survival of mycobacteria in sputum at different temperatures. Czech Med 1981;4:203-8

116 Tessema B, Beer J, Emmrich F, et al. Rate of recovery of Mycobacterium tuberculosis from frozen acid-fast-bacillus smear-positive sputum samples subjected to long-term storage in Northwest Ethiopia. J Clin Microbiol 2011:49:2557-61.

117 von Reyn CF, Horsburgh CR, Olivier KN, et al. Skin test reactions to Mycobacterium tuberculosis purified protein derivative and Mycobacterium avium sensitin among health care workers and medical students in the United States. Int J Tuberc Lung Dis 2001;5:1122-8

118 Edwards LB, Acquaviva FA, Livesay VT, et al. An atlas of sensitivity to tuberculin, PPD-B, and histoplasmin in the United States. Am Rev Respir Dis 1969;99:Suppl:1-132.

119 Stavri H, Ulea I, Radu DL, et al. Serodiagnosis of environmental mycobacterial infections. J Microbiol Methods 2011;86:283-90.

120 Doucette K, Fishman JA. Nontuberculous mycobacterial infection in hematopoietic stem cell and solid organ transplant recipients. Clin Infect Dis 2004:38:1428-39.

121 Kitada S, Kobayashi K, Ichiyama S, et al. Serodiagnosis of Mycobacterium aviumcomplex pulmonary disease using an enzyme immunoassay kit. Am J Respir Crit Care Med 2008;177:793-7

122 Kobayashi K. Serodiagnosis of Mycobacterium avium complex disease in humans: translational research from basic mycobacteriology to clinical medicine. Jpn J Infect Dis 2014:67:329-32.

123 Ferroni A, Sermet-Gaudelus I, Le Bourgeois M, et al. Measurement of immunoglobulin $\mathrm{G}$ against Mycobacterial antigen $\mathrm{A} 60$ in patients with cystic fibrosis and lung infection due to Mycobacterium abscessus. Clin Infect Dis 2005:40:58-66.

124 Steingart KR, Henry M, Ng V, et al. Fluorescence versus conventional sputum smear microscopy for tuberculosis: a systematic review. Lancet Infect Dis 2006;6:570-81.

125 Ulukanligil M, Aslan G, Tasçi S. A comparative study on the different staining methods and number of specimens for the detection of acid fast bacilli. Mem Inst Oswaldo Cruz 2000:95:855-8.

126 Wright PW, Wallace RJ, Wright NW, et al. Sensitivity of fluorochrome microscopy for detection of Mycobacterium tuberculosis versus nontuberculous mycobacteria. J Clin Microbiol 1998;36:1046-9.

127 Murray SJ, Barrett A, Magee JG, et al. Optimisation of acid fast smears for the direct detection of mycobacteria in clinical samples. J Clin Pathol 2003;56:613-5.

128 Kim S, Park EM, Kwon OJ, et al. Direct application of the PCR restriction analysis method for identifying NTM species in AFB smear-positive respiratory specimens. Int J Tuberc Lung Dis 2008;12:1344-6.

129 Franco-Alvarez de Luna F, Ruiz P, Gutiérrez J, et al. Evaluation of the GenoType Mycobacteria Direct assay for detection of Mycobacterium tuberculosis complex and four atypical mycobacterial species in clinical samples. J Clin Microbiol 2006:44:3025-7.

130 Seagar AL, Prendergast C, Emmanuel FX, et al. Evaluation of the GenoType Mycobacteria Direct assay for the simultaneous detection of the Mycobacterium tuberculosis complex and four atypical mycobacterial species in smear-positive respiratory specimens. J Med Microbio/ 2008;57:605-11.

131 Perry MD, White PL, Ruddy M. Potential for use of the Seegene Anyplex MTB/ NTM real-time detection assay in a regional reference laboratory. J Clin Microbiol 2014; 52:1708-10. 
132 Buijtels PC, Petit PL. Comparison of $\mathrm{NaOH}-\mathrm{N}$-acetyl cysteine and sulfuric acid decontamination methods for recovery of mycobacteria from clinical specimens. J Microbiol Methods 2005;62:83-8.

133 Bange FC, Böttger EC. Improved decontamination method for recovering mycobacteria from patients with cystic fibrosis. Eur J Clin Microbiol Infect Dis 2002;21:546-8.

134 Brown-Elliott BA, Griffith DE, Wallace RJ. Diagnosis of nontuberculous mycobacterial infections. Clin Lab Med 2002;22:911-25.

135 Whittier S, Olivier K, Gilligan P, et al. Proficiency testing of clinical microbiology laboratories using modified decontamination procedures for detection of nontuberculous mycobacteria in sputum samples from cystic fibrosis patients. The Nontuberculous Mycobacteria in Cystic Fibrosis Study Group. J Clin Microbiol 1997;35:2706-8.

136 van Ingen J. Microbiological diagnosis of nontuberculous mycobacterial pulmonary disease. Clin Chest Med 2015;36:43-54.

137 Esther CR, Hoberman S, Fine J, et al. Detection of rapidly growing mycobacteria in routine cultures of samples from patients with cystic fibrosis. J Clin Microbiol 2011;49:1421-5

138 Eltringham I, Pickering J, Gough H, et al. Comparison of Mycobacterial Growth Indicator Tube with Culture on RGM Selective Agar for Detection of Mycobacteria in Sputum Samples from Patients with Cystic Fibrosis. J Clin Microbiol 2016:54:2047-50.

139 Preece CL, Perry A, Gray B, et al. A novel culture medium for isolation of rapidlygrowing mycobacteria from the sputum of patients with cystic fibrosis. J Cyst Fibros 2016;15:186-91

140 Hanna BA, Ebrahimzadeh A, Elliott LB, et al. Multicenter evaluation of the BACTEC MGIT 960 system for recovery of mycobacteria. J Clin Microbiol 1999:37:748-52.

141 Wagner D, Vos M, Buiting AG, et al. "Mycobacterium tilburgii" infections. Emerg Infect Dis 2006:12:532-4.

142 Standards Unit MS. UK Standards for Microbiology Investigations. Investigation of specifiments for Mycobacteriam species B40: PHE 2014.

143 Brown-Elliott BA, Wallace RJ. Clinical and taxonomic status of pathogenic nonpigmented or late-pigmenting rapidly growing mycobacteria. Clin Microbiol Rev 2002;15:716-46.

144 Koh WJ, Jeon K, Lee NY, et al. Clinical significance of differentiation of Mycobacterium massiliense from Mycobacterium abscessus. Am J Respir Crit Care Med 2011;183:405-10.

145 Choi GE, Shin SJ, Won CJ, et al. Macrolide treatment for Mycobacterium abscessus and Mycobacterium massiliense infection and inducible resistance. Am J Respir Crit Care Med 2012;186:917-25.

$146 \mathrm{Kim} \mathrm{HY,} \mathrm{Kim} \mathrm{BJ,} \mathrm{Kook} \mathrm{Y,} \mathrm{et} \mathrm{al.} \mathrm{Mycobacterium} \mathrm{massiliense} \mathrm{is} \mathrm{differentiated} \mathrm{from}$ Mycobacterium abscessus and Mycobacterium bolletii by erythromycin ribosome methyltransferase gene (erm) and clarithromycin susceptibility patterns. Microbiol Immunol 2010;54:347-53.

147 Saleeb PG, Drake SK, Murray PR, et al. Identification of mycobacteria in solidculture media by matrix-assisted laser desorption ionization-time of flight mass spectrometry. J Clin Microbiol 2011:49:1790-4.

148 Lotz A, Ferroni A, Beretti JL, et al. Rapid identification of mycobacterial whole cells in solid and liquid culture media by matrix-assisted laser desorption ionization-time of flight mass spectrometry. J Clin Microbiol 2010;48:4481-6.

149 Pignone M, Greth KM, Cooper J, et al. Identification of mycobacteria by matrixassisted laser desorption ionization-time-of-flight mass spectrometry. J Clin Microbio/ 2006; 44:1963-70

150 El Khéchine A, Couderc C, Flaudrops C, et al. Matrix-assisted laser desorption/ ionization time-of-flight mass spectrometry identification of mycobacteria in routine clinical practice. PLoS One 2011;6:e24720.

151 Bille E, Dauphin B, Leto J, et al. MALDI-TOF MS Andromas strategy for the routine identification of bacteria, mycobacteria, yeasts, Aspergillus spp. and positive blood cultures. Clin Microbiol Infect 2012;18:1117-25.

152 Fangous MS, Mougari F, Gouriou S, et al. Classification algorithm for subspecies identification within the Mycobacterium abscessus species, based on matrix-assisted laser desorption ionization-time of flight mass spectrometry. J Clin Microbiol 2014:52:3362-9.

153 Rodríguez-Sánchez B, Ruiz-Serrano MJ, Marín M, et al. Evaluation of Matrix-Assisted Laser Desorption Ionization-Time of Flight Mass Spectrometry for Identification of Nontuberculous Mycobacteria from Clinical Isolates. J Clin Microbiol 2015;53:2737-40.

154 Suffys PN, da Silva Rocha A, de Oliveira M, et al. Rapid identification of Mycobacteria to the species level using INNO-LiPA Mycobacteria, a reverse hybridization assay. J Clin Microbiol 2001;39:4477-82.

155 Lebrun L, Gönüllü N, Boutros N, et al. Use of INNO-LIPA assay for rapid identification of mycobacteria. Diagn Microbiol Infect Dis 2003;46:151-3.

156 Tortoli E, Mariottini A, Mazzarelli G. Evaluation of INNO-LiPA MYCOBACTERIA V2: improved reverse hybridization multiple DNA probe assay for mycobacterial identification. J Clin Microbiol 2003:41:4418-20.

157 Lebrun L, Weill FX, Lafendi L, et al. Use of the INNO-LiPA-MYCOBACTERIA assay (version 2) for identification of Mycobacterium avium-Mycobacterium intracellulareMycobacterium scrofulaceum complex isolates. J Clin Microbiol 2005;43:2567-74.
158 Telenti A, Marchesi F, Balz M, et al. Rapid identification of mycobacteria to the species level by polymerase chain reaction and restriction enzyme analysis. J Clin Microbiol 1993;31:175-8.

159 Devallois A, Goh KS, Rastogi N. Rapid identification of mycobacteria to species level by PCR-restriction fragment length polymorphism analysis of the hsp65 gene and proposition of an algorithm to differentiate 34 mycobacterial species. J Clin Microbiol 1997;35:2969-73.

160 Leao SC, Tortoli E, Euzéby JP, et al. Proposal that Mycobacterium massiliense and Mycobacterium bolletii be united and reclassified as Mycobacterium abscessus subsp. bolletii comb. nov., designation of Mycobacterium abscessus subsp. abscessus subsp. nov. and emended description of Mycobacterium abscessus. Int I Syst Evol Microbiol 2011;61:2311-3.

161 Ringuet $\mathrm{H}$, Akoua-Koffi $\mathrm{C}$, Honore $\mathrm{S}$, et al. hsp65 sequencing for identification of rapidly growing mycobacteria. J Clin Microbio/ 1999;37:852-7.

162 Macheras E, Roux AL, Ripoll F, et al. Inaccuracy of single-target sequencing for discriminating species of the Mycobacterium abscessus group. J Clin Microbiol 2009:47:2596-600.

163 Kim K, Hong SH, Kim BJ, et al. Separation of Mycobacterium abscessus into subspecies or genotype level by direct application of peptide nucleic acid multi-probe- real-time PCR method into sputa samples. BMC Infect Dis 2015; 15:325.

164 Macheras E, Roux AL, Bastian S, et al. Multilocus sequence analysis and rpoB sequencing of Mycobacterium abscessus (sensu lato) strains. J Clin Microbiol 2011;49:491-9.

165 Macheras E, Konjek J, Roux AL, et al. Multilocus sequence typing scheme for the Mycobacterium abscessus complex. Res Microbiol 2014;165:82-90.

166 Hoffner SE, Svenson SB, Källenius G. Synergistic effects of antimycobacterial drug combinations on Mycobacterium avium complex determined radiometrically in liquid medium. Eur J Clin Microbio/ 1987:6:530-5.

167 Banks J, Jenkins PA. Combined versus single antituberculosis drugs on the in vitro sensitivity patterns of non-tuberculous mycobacteria. Thorax 1987;42:838-42.

168 Heifets LB. Synergistic effect of rifampin, streptomycin, ethionamide, and ethambutol on Mycobacterium intracellulare. Am Rev Respir Dis 1982;125:43-8.

169 Woods GL, Brown-Elliott BA, Conville PS, et al. M24 Susceptibility Testing of Mycobacteria Nocardiae, and Other Aerobic Actinomycetes; Approved Standard. 2nd Edition, 2011.

170 van Ingen J, Egelund EF, Levin A, et al. The pharmacokinetics and pharmacodynamics of pulmonary Mycobacterium avium complex disease treatment. Am J Respir Crit Care Med 2012;186:559-65.

171 van Ingen J, Boeree MJ, van Soolingen D, et al. Resistance mechanisms and drug susceptibility testing of nontuberculous mycobacteria. Drug Resist Updat 2012;15:149-61.

172 Chaisson RE, Benson CA, Dube MP, et al. Clarithromycin therapy for bacteremic Mycobacterium avium complex disease. A randomized, double-blind, dose-ranging study in patients with AIDS. AIDS Clinical Trials Group Protocol 157 Study Team. Ann Intern Med 1994;121:905-11.

173 Wallace RJ, Brown BA, Griffith DE, et al. Clarithromycin regimens for pulmonary Mycobacterium avium complex. The first 50 patients. Am J Respir Crit Care Med 1996;153:1766-72.

174 Tanaka E, Kimoto T, Tsuyuguchi K, et al. Effect of clarithromycin regimen for Mycobacterium avium complex pulmonary disease. Am J Respir Crit Care Med 1999; 160:866-72.

175 Griffith DE, Brown-Elliott BA, Langsjoen B, et al. Clinical and molecular analysis of macrolide resistance in Mycobacterium avium complex lung disease. Am J Respir Crit Care Med 2006:174:928-34.

176 Morimoto K, Namkoong H, Hasegawa N, et al. Macrolide-Resistant Mycobacterium avium Complex Lung Disease: Analysis of 102 Consecutive Cases. Ann Am Thorac Soc 2016;13:1904-11.

177 Brown-Elliott BA, lakhiaeva E, Griffith DE, et al. In vitro activity of amikacin against isolates of Mycobacterium avium complex with proposed MIC breakpoints and finding of a 16S rRNA gene mutation in treated isolates. J Clin Microbiol 2013:51:3389-94.

178 Sison JP, Yao Y, Kemper CA, et al. Treatment of Mycobacterium avium complex infection: do the results of in vitro susceptibility tests predict therapeutic outcome in humans? J Infect Dis 1996:173:677-83.

179 Ahn CH, Wallace RJ, Steele LC, et al. Sulfonamide-containing regimens for disease caused by rifampin-resistant Mycobacterium kansasii. Am Rev Respir Dis 1987;135:10-16.

180 Wallace RJ, Dunbar D, Brown BA, et al. Rifampin-resistant Mycobacterium kansasii. Clin Infect Dis 1994; 18:736-43

181 van Ingen J, Totten SE, Heifets LB, et al. Drug susceptibility testing and pharmacokinetics question current treatment regimens in Mycobacterium simiae complex disease. Int J Antimicrob Agents 2012;39:173-6.

182 Wallace RJ, Swenson JM, Silcox VA, et al. Treatment of nonpulmonary infections due to Mycobacterium fortuitum and Mycobacterium chelonei on the basis of in vitro susceptibilities. J Infect Dis 1985:152:500-14.

183 Jarand J, Levin A, Zhang L, et al. Clinical and microbiologic outcomes in patients receiving treatment for Mycobacterium abscessus pulmonary disease. Clin Infect Dis 2011;52:565-71 
184 Prajapati S, Upadhyay K, Mukherjee A, et al. High prevalence of primary drug resistance in children with intrathoracic tuberculosis in India. Paediatr Int Child Health 2016;36:0000000041.

185 Griffith DE, Adjemian J, Brown-Elliott BA, et al. Semiquantitative Culture Analysis during Therapy for Mycobacterium avium Complex Lung Disease. Am J Respir Crit Care Med 2015;192:754-60.

186 Ikedo Y. The significance of bronchoscopy for the diagnosis of Mycobacterium avium complex (MAC) pulmonary disease. Kurume Med J 2001:48:15-19.

187 Huang JH, Kao PN, Adi V, et al. Mycobacterium avium-intracellulare pulmonary infection in HIV-negative patients without preexisting lung disease: diagnostic and management limitations. Chest 1999;115:1033-40.

188 Ahn CH, McLarty JW, Ahn SS, et al. Diagnostic criteria for pulmonary disease caused by Mycobacterium kansasii and Mycobacterium intracellulare. Am Rev Respir Dis 1982;125:388-91.

189 Lam PK, Griffith DE, Aksamit TR, et al. Factors related to response to intermittent treatment of Mycobacterium avium complex lung disease. Am J Respir Crit Care Med 2006:173:1283-9.

190 Hollings NP, Wells AU, Wilson R, et al. Comparative appearances of non-tuberculous mycobacteria species: a CT study. Eur Radiol 2002;12:2211-7.

191 Moore EH. Atypical mycobacterial infection in the lung: CT appearance. Radiology 1993; 187:777-82.

192 McEvoy S, Lavelle L, Kilcoyne A, et al. High-resolution CT of nontuberculous mycobacterium infection in adult CF patients: diagnostic accuracy. Eur Radiol 2012:22:2736-42

193 Obayashi Y, Fujita J, Suemitsu I, et al. Successive follow-up of chest computed tomography in patients with Mycobacterium avium-intracellulare complex. Respir Med 1999;93:11-15

194 Maycher B, O'Connor R, Long R. Computed tomographic abnormalities in Mycobacterium avium complex lung disease include the mosaic pattern of reduced lung attenuation. Can Assoc Radiol J 2000;51:93-102.

195 Ferrara I, Cappabianca S, Brunese L, et al. HRCT in detection of pulmonary infections from nontuberculous mycobacteria: personal experience. Radiol Med 2009:114:376-89.

196 Lynch DA, Simone PM, Fox MA, et al. CT features of pulmonary Mycobacterium avium complex infection. J Comput Assist Tomogr 1995:19:353-60.

197 Kitada S, Uenami T, Yoshimura K, et al. Long-term radiographic outcome of nodular bronchiectatic Mycobacterium avium complex pulmonary disease. Int J Tuberc Lung Dis 2012;16:660-4

198 Zoumot Z, Boutou AK, Gill SS, et al. Mycobacterium avium complex infection in noncystic fibrosis bronchiectasis. Respirology 2014;19:714-22.

199 Primack SL, Logan PM, Hartman TE, et al. Pulmonary tuberculosis and Mycobacterium avium-intracellulare: a comparison of CT findings. Radiology 1995:194:413-7.

200 Ushiki A, Yamazaki Y, Koyama S, et al. Bronchoscopic microsampling for bacterial colony counting in relevant lesions in patients with pulmonary Mycobacterium avium complex infection. Intern Med 2011:50:1287-92.

201 Hayashi M, Takayanagi N, Kanauchi T, et al. Prognostic factors of 634 HIV-negative patients with Mycobacterium avium complex lung disease. Am J Respir Crit Care Med 2012;185:575-83.

202 Shu CC, Lee CH, Hsu CL, et al. Clinical characteristics and prognosis of nontuberculous mycobacterial lung disease with different radiographic patterns. Lung 2011;189:467-74.

203 Kuroishi S, Nakamura Y, Hayakawa H, et al. Mycobacterium avium complex disease: prognostic implication of high-resolution computed tomography findings. Eur Respir J 2008:32:147-52

204 Lee G, Lee KS, Moon JW, et al. Nodular bronchiectatic Mycobacterium avium complex pulmonary disease. Natural course on serial computed tomographic scans. Ann Am Thorac Soc 2013;10:299-306.

205 Takeda K, Imamura Y, Takazono T, et al. The risk factors for developing of chronic pulmonary aspergillosis in nontuberculous mycobacteria patients and clinical characteristics and outcomes in chronic pulmonary aspergillosis patients coinfected with nontuberculous mycobacteria. Med Mycol 2016;54:120-7.

206 Jeong BH, Kim SY, Jeon K, et al. Serodiagnosis of Mycobacterium avium complex and Mycobacterium abscessus complex pulmonary disease by use of $\lg \mathrm{A}$ antibodies to glycopeptidolipid core antigen. J Clin Microbiol 2013;51:2747-9.

207 Kitada S, Levin A, Hiserote M, et al. Serodiagnosis of Mycobacterium avium complex pulmonary disease in the USA. Eur Respir J 2013;42:454-60.

208 Shu CC, Ato M, Wang JT, et al. Sero-diagnosis of Mycobacterium avium complex lung disease using serum immunoglobulin A antibody against glycopeptidolipid antigen in Taiwan. PLoS One 2013:8:e80473.

209 Qvist T, Pressler T, Taylor-Robinson D, et al. Serodiagnosis of Mycobacterium abscessus complex infection in cystic fibrosis. Eur Respir J 2015;46:707-16.

210 Huebner RE, Schein MF, Cauthen GM, et al. Evaluation of the clinical usefulness of mycobacterial skin test antigens in adults with pulmonary mycobacterioses. Am Rev Respir Dis 1992:145:1160-6.

211 Ra SW, Lyu J, Choi CM, et al. Distinguishing tuberculosis from Mycobacterium avium complex disease using an interferon-gamma release assay. Int J Tuberc Lung Dis 2011;15:635-40
212 Demura Y, Tsuchida T, Uesaka D, et al. Usefulness of 18F-fluorodeoxyglucose positron emission tomography for diagnosing disease activity and monitoring therapeutic response in patients with pulmonary mycobacteriosis. Eur J Nud Med Mol Imaging 2009;36:632-9.

213 Research Committee of the British Thoracic Society. First randomised trial of treatments for pulmonary disease caused by $\mathrm{M}$ avium intracellulare, $\mathrm{M}$ malmoense, and $\mathrm{M}$ xenopi in HIV negative patients: rifampicin, ethambutol and isoniazid versus rifampicin and ethambutol. Thorax 2001;56:167-72.

214 Jenkins PA, Campbell IA, Banks J, et al. Clarithromycin vs ciprofloxacin as adjuncts to rifampicin and ethambutol in treating opportunist mycobacterial lung diseases and an assessment of Mycobacterium vaccae immunotherapy. Thorax 2008;63:627-34.

215 Roux AL, Catherinot E, Ripoll F, et al. Multicenter study of prevalence of nontuberculous mycobacteria in patients with cystic fibrosis in france. J Clin Microbiol 2009:47:4124-8

216 Harada T, Akiyama Y, Kurashima A, et al. Clinical and microbiological differences between Mycobacterium abscessus and Mycobacterium massiliense lung diseases. J Clin Microbiol 2012;50:3556-61.

217 Lyu J, Jang HJ, Song JW, et al. Outcomes in patients with Mycobacterium abscessus pulmonary disease treated with long-term injectable drugs. Respir Med 2011;105:781-7.

218 Boyle DP, Zembower TR, Reddy S, et al. Comparison of Clinical Features, Virulence, and Relapse among Mycobacterium avium Complex Species. Am J Respir Crit Care Med 2015:191:1310-7.

219 Koh WJ, Jeong BH, Jeon $\mathrm{K}$, et al. Clinical significance of the differentiation between Mycobacterium avium and Mycobacterium intracellulare in $\mathrm{M}$ avium complex lung disease. Chest 2012;142:1482-8

220 Kim SY, Lee ST, Jeong BH, et al. Clinical significance of mycobacterial genotyping in Mycobacterium avium lung disease in Korea. Int J Tuberc Lung Dis 2012:16:1393-9.

221 Kikuchi T, Kobashi Y, Hirano T, et al. Mycobacterium avium genotype is associated with the therapeutic response to lung infection. Clin Microbiol Infect 2014;20:256-62.

222 Shin SJ, Choi GE, Cho SN, et al. Mycobacterial genotypes are associated with clinical manifestation and progression of lung disease caused by Mycobacterium abscessus and Mycobacterium massiliense. Clin Infect Dis 2013;57:32-9.

223 Ito Y, Hirai T, Maekawa K, et al. Predictors of 5-year mortality in pulmonary Mycobacterium avium-intracellulare complex disease. Int J Tuberc Lung Dis 2012;16:408-14.

224 Kitada S, Maekura R, Toyoshima N, et al. Use of glycopeptidolipid core antigen for serodiagnosis of mycobacterium avium complex pulmonary disease in immunocompetent patients. Clin Diagn Lab Immunol 2005;12:44-51.

225 Kitada S, Nishiuchi Y, Hiraga T, et al. Serological test and chest computed tomography findings in patients with Mycobacterium avium complex lung disease. Eur Respir J 2007;29:1217-23.

226 Russell CD, Claxton P, Doig C, et al. Non-tuberculous mycobacteria: a retrospective review of Scottish isolates from 2000 to 2010. Thorax 2014;69:593-5.

227 Kobashi Y, Matsushima T, Oka M. A double-blind randomized study of aminoglycoside infusion with combined therapy for pulmonary Mycobacterium avium complex disease. Respir Med 2007:101:130-8.

228 Tsukamura M, Ichiyama S, Miyachi T. Superiority of enviomycin or streptomycin over ethambutol in initial treatment of lung disease caused by Mycobacterium avium complex. Chest 1989;95:1056-8.

229 Ahn CH, Ahn SS, Anderson RA, et al. A four-drug regimen for initial treatment of cavitary disease caused by Mycobacterium avium complex. Am Rev Respir Dis 1986;134:438-41.

230 Dutt AK, Stead WW. Long-term results of medical treatment in Mycobacterium intracellulare infection. Am J Med 1979;67:449-53.

231 Fujita M, Kajiki A, Tao Y, et al. The clinical efficacy and safety of a fluoroquinolonecontaining regimen for pulmonary MAC disease. J Infect Chemother 2012:18:146-51.

232 Miwa S, Shirai M, Toyoshima M, et al. Efficacy of clarithromycin and ethambutol for Mycobacterium avium complex pulmonary disease. A preliminary study. Ann Am Thorac Soc 2014;11:23-9.

$233 \mathrm{Sim}$ YS, Park HY, Jeon K, et al. Standardized combination antibiotic treatment of Mycobacterium avium complex lung disease. Yonsei Med J 2010;51:888-94.

234 Kobashi Y, Matsushima T. The microbiological and clinical effects of combined therapy according to guidelines on the treatment of pulmonary Mycobacterium avium complex disease in Japan - including a follow-up study. Respiration 2007;74:394-400.

235 Griffith DE, Brown BA, Girard WM, et al. Azithromycin-containing regimens for treatment of Mycobacterium avium complex lung disease. Clin Infect Dis 2001;32:1547-53.

236 Griffith DE, Brown BA, Cegielski P, et al. Early results (at 6 months) with intermittent clarithromycin-including regimens for lung disease due to Mycobacterium avium complex. Clin Infect Dis 2000;30:288-92.

237 Wallace RJ, Brown-Elliott BA, McNulty S, et al. Macrolide/Azalide therapy for nodular/ bronchiectatic mycobacterium avium complex lung disease. Chest 2014;146:276-82.

238 Jeong $\mathrm{BH}$, Jeon $\mathrm{K}$, Park HY, et al. Intermittent antibiotic therapy for nodular bronchiectatic Mycobacterium avium complex lung disease. Am J Respir Crit Care Med 2015;191:96-103. 
239 Koh WJ, Jeong BH, Jeon K, et al. Response to Switch from Intermittent Therapy to Daily Therapy for Refractory Nodular Bronchiectatic Mycobacterium avium Complex Lung Disease. Antimicrob Agents Chemother 2015;59:4994-6.

240 Kobashi Y, Abe M, Mouri K, et al. Relationship between clinical efficacy for pulmonary MAC and drug-sensitivity test for isolated MAC in a recent 6-year period. J Infect Chemother 2012;18:436-43.

241 Hasegawa N, Nishimura T, Ohtani S, et al. Therapeutic effects of various initial combinations of chemotherapy including clarithromycin against Mycobacterium avium complex pulmonary disease. Chest 2009;136:1569-75.

242 Kobashi Y, Matsushima T. The effect of combined therapy according to the guidelines for the treatment of Mycobacterium avium complex pulmonary disease. Intern Med 2003;42:670-5.

243 Griffith DE, Brown BA, Girard WM, et al. Azithromycin activity against Mycobacterium avium complex lung disease in patients who were not infected with human immunodeficiency virus. Clin Infect Dis 1996:23:983-9.

244 Wallace RJ, Brown BA, Griffith DE. Drug intolerance to high-dose clarithromycin among elderly patients. Diagn Microbiol Infect Dis 1993;16:215-21.

245 Griffith DE, Brown BA, Girard WM, et al. Adverse events associated with high-dose rifabutin in macrolide-containing regimens for the treatment of Mycobacterium avium complex lung disease. Clin Infect Dis 1995;21:594-8.

246 Wallace RJ, Brown BA, Griffith DE, et al. Initial clarithromycin monotherapy for Mycobacterium avium-intracellulare complex lung disease. Am J Respir Crit Care Med 1994;149:1335-41.

247 Brown BA, Griffith DE, Girard W, et al. Relationship of adverse events to serum drug levels in patients receiving high-dose azithromycin for mycobacterial lung disease. Clin Infect Dis 1997;24:958-64.

248 Griffith DE, Brown BA, Murphy DT, et al. Initial (6-month) results of three-times weekly azithromycin in treatment regimens for Mycobacterium avium complex lung disease in human immunodeficiency virus-negative patients. J Infect Dis 1998;178:121-6.

249 Roussel G, Igual J. Clarithromycin with minocycline and clofazimine for Mycobacterium avium intracellulare complex lung disease in patients without the acquired immune deficiency syndrome. GETIM. Groupe d'Etude et de Traitement des Infections à Mycobactéries. Int J Tuberc Lung Dis 1998;2:462-70.

250 Davidson PT, Khanijo V, Goble M, et al. Treatment of disease due to Mycobacterium intracellulare. Rev Infect Dis 1981;3:1052-9.

251 Hunter AM, Campbell IA, Jenkins PA, et al. Treatment of pulmonary infections caused by mycobacteria of the Mycobacterium avium-intracellulare complex. Thorax 1981:36:326-9.

252 Jo KW, Kim S, Lee JY, et al. Treatment outcomes of refractory MAC pulmonary disease treated with drugs with unclear efficacy. J Infect Chemother 2014;20:602-6.

253 Koh WJ, Hong G, Kim SY, et al. Treatment of refractory Mycobacterium avium complex lung disease with a moxifloxacin-containing regimen. Antimicrob Agents Chemother 2013:57:2281-5.

254 Field SK, Cowie RL. Treatment of Mycobacterium avium-intracellulare complex lung disease with a macrolide, ethambutol, and clofazimine. Chest 2003; 124:1482-6.

255 Winthrop KL, Ku JH, Marras TK, et al. The tolerability of linezolid in the treatment of nontuberculous mycobacterial disease. Eur Respir J 2015;45:1177-9.

256 Philley JV, Wallace RJ, Benwill JL, et al. Preliminary Results of Bedaquiline as Salvage Therapy for Patients With Nontuberculous Mycobacterial Lung Disease. Chest 2015; 148:499-506

257 Jarand J, Davis JP, Cowie RL, et al. Long-term Follow-up of Mycobacterium avium Complex Lung Disease in Patients Treated With Regimens Including Clofazimine and/ or Rifampin. Chest 2016:149:1285-93.

258 Davis KK, Kao PN, Jacobs SS, et al. Aerosolized amikacin for treatment of pulmonary Mycobacterium avium infections: an observational case series. BMC Pulm Med 2007;7:2.

259 Olivier KN, Shaw PA, Glaser TS, et al. Inhaled amikacin for treatment of refractory pulmonary nontuberculous mycobacterial disease. Ann Am Thorac Soc 2014;11:30-5.

260 Olivier KN, Griffith DE, Eagle G, et al. Randomized Trial of Liposomal Amikacin for Inhalation in Nontuberculous Mycobacterial Lung Disease. Am J Respir Crit Care Med 2017; 195:814-823

261 van Ingen J, Totten SE, Helstrom NK, et al. In vitro synergy between clofazimine and amikacin in treatment of nontuberculous mycobacterial disease. Antimicrob Agents Chemother 2012;56:6324-7.

262 Jenkins PA, Banks J, Campbell IA, et al. Mycobacterium kansasii pulmonary infection: a prospective study of the results of nine months of treatment with rifampicin and ethambutol. Research Committee, British Thoracic Society. Thorax 1994:49:442-5.

263 Ahn CH, Lowell JR, Ahn SS, et al. Short-course chemotherapy for pulmonary disease caused by Mycobacterium kansasii. Am Rev Respir Dis 1983;128:1048-50.

264 Sauret J, Hernández-Flix S, Castro E, et al. Treatment of pulmonary disease caused by Mycobacterium kansasii: results of 18 vs 12 months' chemotherapy. Tuber Lung Dis 1995:76:104-8.

265 Griffith DE, Brown-Elliott BA, Wallace RJ. Thrice-weekly clarithromycin-containing regimen for treatment of Mycobacterium kansasii lung disease: results of a preliminary study. Clin Infect Dis 2003;37:1178-82.
266 Ahn CH, Lowell JR, Ahn SS, et al. Chemotherapy for pulmonary disease due to Mycobacterium kansasii: efficacies of some individual drugs. Rev Infect Dis 1981;3:1028-34

267 Pezzia W, Raleigh JW, Bailey MC, et al. Treatment of pulmonary disease due to Mycobacterium kansasii: recent experience with rifampin. Rev Infect Dis 1981;3:1035-9.

268 Banks J, Hunter AM, Campbell IA, et al. Pulmonary infection with Mycobacterium kansasii in Wales, 1970-9: review of treatment and response. Thorax 1983;38:271-4

269 Evans AJ, Crisp AJ, Hubbard RB, et al. Pulmonary Mycobacterium kansasii infection: comparison of radiological appearances with pulmonary tuberculosis. Thorax 1996;51:1243-7.

270 Santin M, Dorca J, Alcaide F, et al. Long-term relapses after 12-month treatment for Mycobacterium kansasii lung disease. Eur Respir J 2009;33:148-52.

271 Park HK, Koh WJ, Shim TS, et al. Clinical characteristics and treatment outcomes of Mycobacterium kansasii lung disease in Korea. Yonsei Med J 2010;51:552-6.

272 Shitrit D, Baum GL, Priess R, et al. Pulmonary Mycobacterium kansasii infection in Israel, 1999-2004: clinical features, drug susceptibility, and outcome. Chest 2006;129:771-6.

273 Banks J, Jenkins PA, Smith AP. Pulmonary infection with Mycobacterium malmoense-a review of treatment and response. Tubercle 1985;66:197-203.

274 Murray MP, Laurenson IF, Hill AT. Outcomes of a standardized triple-drug regimen for the treatment of nontuberculous mycobacterial pulmonary infection. Clin Infect Dis 2008;47:222-4

275 Hoefsloot W, van Ingen J, de Lange WC, et al. Clinical relevance of Mycobacterium malmoense isolation in The Netherlands. Eur Respir J 2009:34:926-31.

276 Varadi RG, Marras TK. Pulmonary Mycobacterium xenopi infection in non-HIVinfected patients: a systematic review. Int J Tuberc Lung Dis 2009:13:1210-8.

277 Costrini AM, Mahler DA, Gross WM, et al. Clinical and roentgenographic features of nosocomial pulmonary disease due to Mycobacterium xenopi. Am Rev Respir Dis 1981;123:104-9.

278 Smith MJ, Citron KM. Clinical review of pulmonary disease caused by Mycobacterium xenopi. Thorax 1983:38:373-7.

279 Banks J, Hunter AM, Campbell IA, et al. Pulmonary infection with mycobacterium xenopi: review of treatment and response. Thorax 1984;39:376-82.

280 Johnston ID. Mycobacterium xenopi infection and aspergilloma. Tubercle 1988;69:139-43.

281 Contreras MA, Cheung OT, Sanders DE, et al. Pulmonary infection with nontuberculous mycobacteria. Am Rev Respir Dis 1988;137:149-52.

282 Al Jarad N, Demertzis P, Jones DJ, et al. Comparison of characteristics of patients and treatment outcome for pulmonary non-tuberculous mycobacterial infection and pulmonary tuberculosis. Thorax 1996;51:137-9.

283 van Ingen J, Boeree MJ, de Lange WC, et al. Mycobacterium xenopi clinical relevance and determinants, the Netherlands. Emerg Infect Dis 2008;14:385-9.

284 Andréjak C, Lescure FX, Pukenyte $E$, et al. Mycobacterium xenopi pulmonary infections: a multicentric retrospective study of 136 cases in north-east France. Thorax 2009:64:291-6.

285 Jeon K, Kwon OJ, Lee NY, et al. Antibiotic treatment of Mycobacterium abscessus lung disease: a retrospective analysis of 65 patients. Am J Respir Crit Care Med 2009;180:896-902.

286 Maurer FP, Castelberg C, Quiblier C, et al. Erm(41)-dependent inducible resistance to azithromycin and clarithromycin in clinical isolates of Mycobacterium abscessus. $J$ Antimicrob Chemother 2014;69:1559-63.

287 Lyu J, Kim BJ, Kim BJ, et al. A shorter treatment duration may be sufficient for patients with Mycobacterium massiliense lung disease than with Mycobacterium abscessus lung disease. Respir Med 2014;108:1706-12.

288 Wallace RJ, Dukart G, Brown-Elliott BA, et al. Clinical experience in 52 patients with tigecycline-containing regimens for salvage treatment of Mycobacterium abscessus and Mycobacterium chelonae infections. J Antimicrob Chemother 2014;69:1945-53.

289 Shen GH, Wu BD, Hu ST, et al. High efficacy of clofazimine and its synergistic effect with amikacin against rapidly growing mycobacteria. Int J Antimicrob Agents 2010;35:400-4

290 Dubée V, Bernut A, Cortes $M$, et al. $\beta$-Lactamase inhibition by avibactam in Mycobacterium abscessus. J Antimicrob Chemother 2015;70:1051-8.

291 Milanés-Virelles MT, García-García I, Santos-Herrera Y, et al. Adjuvant interferon gamma in patients with pulmonary atypical Mycobacteriosis: a randomized, doubleblind, placebo-controlled study. BMC Infect Dis 2008;8:17.

292 Maekawa K, Ito Y, Oga T, et al. High-resolution computed tomography and healthrelated quality of life in Mycobacterium avium complex disease. Int J Tuberc Lung Dis 2013;17:829-35.

293 Koh WJ, Jeong BH, Jeon K, et al. Therapeutic drug monitoring in the treatment of Mycobacterium avium complex lung disease. Am J Respir Crit Care Med 2012; 186:797-802

294 Magis-Escurra C, Alffenaar JW, Hoefnagels I, et al. Pharmacokinetic studies in patients with nontuberculous mycobacterial lung infections. Int J Antimicrob Agents 2013:42:256-61.

295 Peloquin CA, Berning SE, Nitta AT, et al. Aminoglycoside toxicity: daily versus thrice-weekly dosing for treatment of mycobacterial diseases. Clin Infect Dis 2004;38:1538-44. 
296 Prezant TR, Agapian JV, Bohlman MC, et al. Mitochondrial ribosomal RNA mutation associated with both antibiotic-induced and non-syndromic deafness. Nat Genet 1993:4:289-94.

297 Griffith DE, Brown-Elliott BA, Shepherd S, et al. Ethambutol ocular toxicity in treatment regimens for Mycobacterium avium complex lung disease. Am J Respir Crit Care Med 2005;172:250-3.

298 Varughese A, Brater DC, Benet LZ, et al. Ethambutol kinetics in patients with impaired renal function. Am Rev Respir Dis 1986;134:34-8.

299 Milburn H, Ashman N, Davies P, et al. Guidelines for the prevention and management of Mycobacterium tuberculosis infection and disease in adult patients with chronic kidney disease. Thorax 2010;65:557-70.

300 Ray WA, Murray KT, Hall K, et al. Azithromycin and the risk of cardiovascular death. N Engl J Med 2012;366:1881-90.

301 Marras TK, Prevots DR, Jamieson FB, et al. Opinions differ by expertise in Mycobacterium avium complex disease. Ann Am Thorac Soc 2014;11:17-22.

302 Adjemian J, Prevots DR, Gallagher J, et al. Lack of adherence to evidence-based treatment guidelines for nontuberculous mycobacterial lung disease. Ann Am Thorac Soc 2014;11:9-16.

303 Yu JA, Pomerantz M, Bishop A, et al. Lady Windermere revisited: treatment with thoracoscopic lobectomy/segmentectomy for right middle lobe and lingular bronchiectasis associated with non-tuberculous mycobacterial disease. Eur J Cardiothorac Surg 2011;40:671-5.

304 Corpe RF. Surgical management of pulmonary disease due to Mycobacterium aviumintracellulare. Rev Infect Dis 1981;3:1064-7.

305 Koh WJ, Kim YH, Kwon OJ, et al. Surgical treatment of pulmonary diseases due to nontuberculous mycobacteria. J Korean Med Sci 2008;23:397-401.

306 Law SW. Surgical treatment of atypical mycobacterial disease; a survey of experience in veterans administration hospitals. Dis Chest 1965:47:296-303.

307 Mitchell JD, Bishop A, Cafaro A, et al. Anatomic lung resection for nontuberculous mycobacterial disease. Ann Thorac Surg 2008;85:1887-93.

308 Moran JF, Alexander LG, Staub EW, et al. Long-term results of pulmonary resection for atypical mycobacterial disease. Ann Thorac Surg 1983;35:597-604.

309 Muangsombut J, Hankins JR, Miller JE, et al. Surgical treatment of pulmonary infections caused by atypical mycobacteria. Am Surg 1975;41:37-40.

310 Nelson KG, Griffith DE, Brown BA, et al. Results of operation in Mycobacterium avium-intracellulare lung disease. Ann Thorac Surg 1998;66:325-9.

311 Ono N, Satoh K, Yokomise H, et al. Surgical management of Mycobacterium avium complex disease. Thorac Cardiovasc Surg 1997;45:311-3.

312 Parrot RG, Grosset JH. Post-surgical outcome of 57 patients with Mycobacterium xenopi pulmonary infection. Tubercle 1988;69:47-55.

313 Shiraishi Y, Fukushima K, Komatsu H, et al. Early pulmonary resection for localized Mycobacterium avium complex disease. Ann Thorac Surg 1998;66:183-6.

314 Shiraishi Y, Nakajima Y, Takasuna K, et al. Surgery for Mycobacterium avium complex lung disease in the clarithromycin era. Eur $J$ Cardiothorac Surg 2002;21:314-8.

315 Shiraishi Y, Katsuragi $\mathrm{N}$, Kita $\mathrm{H}$, et al. Different morbidity after pneumonectomy: multidrug-resistant tuberculosis versus non-tuberculous mycobacterial infection. Interact Cardiovasc Thorac Surg 2010;11:429-32.

316 Tsunezuka Y, Sato H, Hiranuma C. Surgical outcome of mycobacterium other than mycobacterium tuberculosis pulmonary disease. Thorac Cardiovasc Surg 2000:48:290-3.

317 van Ingen J, Verhagen AF, Dekhuijzen PN, et al. Surgical treatment of nontuberculous mycobacterial lung disease: strike in time. Int J Tuberc Lung Dis 2010;14:99-105.
318 Watanabe M, Hasegawa N, Ishizaka A, et al. Early pulmonary resection for Mycobacterium avium complex lung disease treated with macrolides and quinolones. Ann Thorac Surg 2006;81:2026-30.

319 Zvetina JR, Neville WE, Maben HC, et al. Surgical treatment of pulmonary disease due to Mycobacterium kansasii. Ann Thorac Surg 1971;11:551-6.

320 Kang HK, Park HY, Kim D, et al. Treatment outcomes of adjuvant resectional surgery for nontuberculous mycobacterial lung disease. BMC Infect Dis 2015;15:76.

321 Shiraishi $\mathrm{Y}$, Katsuragi N, Kita $\mathrm{H}$, et al. Adjuvant surgical treatment of nontuberculous mycobacterial lung disease. Ann Thorac Surg 2013;96:287-91.

322 Wang GS, Wang Z, Yang L, et al. Thoracoscopic management for bronchiectasis with non-tuberculous mycobacterial infection. Chin Med J 2008;121:2539-43.

323 Lim E, Baldwin D, Beckles $M$, et al. Guidelines on the radical management of patients with lung cancer. Thorax 2010;65(Suppl 3):iii1-27.

324 Colice GL, Shafazand S, Griffin JP, et al. Physiologic evaluation of the patient with lung cancer being considered for resectional surgery: ACCP evidenced-based clinical practice guidelines (2nd edition). Chest 2007(3 Suppl):161S-77S.

325 Char A, Hopkinson NS, Hansell DM, et al. Evidence of mycobacterial disease in COPD patients with lung volume reduction surgery; the importance of histological assessment of specimens: a cohort study. BMC Pulm Med 2014;14:124.

326 Dorman S, Subramanian A. AST Infectious Diseases Community of Practice. Nontuberculous mycobacteria in solid organ transplant recipients. Am J Transplant 2009;9(Suppl 4):S63-9.

327 Weill D, Benden C, Corris PA, et al. A consensus document for the selection of lung transplant candidates: 2014-an update from the Pulmonary Transplantation Council of the International Society for Heart and Lung Transplantation. J Heart Lung Transplant 2015;34:1-15.

328 Knoll BM, Kappagoda S, Gill RR, et al. Non-tuberculous mycobacterial infection among lung transplant recipients: a 15-year cohort study. Transp/ Infect Dis 2012;14:452-60.

329 Huang HC, Weigt SS, Derhovanessian A, et al. Non-tuberculous mycobacterium infection after lung transplantation is associated with increased mortality. J Heart Lung Transplant 2011;30:790-8.

330 Chalermskulrat W, Sood N, Neuringer IP, et al. Non-tuberculous mycobacteria in end stage cystic fibrosis: implications for lung transplantation. Thorax 2006;61:507-13.

331 Chernenko SM, Humar A, Hutcheon M, et al. Mycobacterium abscessus infections in lung transplant recipients: the international experience. J Heart Lung Transplant 2006;25:1447-55.

332 Robinson PD, Harris KA, Aurora P, et al. Paediatric lung transplant outcomes vary with Mycobacterium abscessus complex species. Eur Respir J 2013;41:1230-2.

333 Gilljam M, Scherstén H, Silverborn M, et al. Lung transplantation in patients with cystic fibrosis and Mycobacterium abscessus infection. J Cyst Fibros 2010;9:272-6.

334 Qvist T, Katzenstein TL, Lillebaek T, et al. First report of lung transplantation in a patient with active pulmonary Mycobacterium simiae infection. Transplant Proc 2013:45:803-5.

335 Lobo LJ, Chang LC, Esther CR, et al. Lung transplant outcomes in cystic fibrosis patients with pre-operative Mycobacterium abscessus respiratory infections. Clin Transplant 2013;27:523-9.

336 Floto RA, Olivier KN, Saiman L, et al. US Cystic Fibrosis Foundation and European Cystic Fibrosis Society consensus recommendations for the management of non-tuberculous mycobacteria in individuals with cystic fibrosis. Thorax 2016;71(Suppl 1):i1-22. 